\title{
Appraisal of self-care agency A.S.A.-scale : reliability and validity testing of the Dutch version of the A.S.A.- scale measuring Orem's concept 'Self-Care Agency'
}

Citation for published version (APA):

Evers, G. C. M. (1989). Appraisal of self-care agency A.S.A.-scale : reliability and validity testing of the Dutch version of the A.S.A.-scale measuring Orem's concept 'Self-Care Agency'. [Doctoral Thesis, Maastricht University]. Van Gorcum. https://doi.org/10.26481/dis.19890629ge

Document status and date:

Published: 01/01/1989

DOI:

10.26481/dis.19890629ge

Document Version:

Publisher's PDF, also known as Version of record

Please check the document version of this publication:

- A submitted manuscript is the version of the article upon submission and before peer-review. There can be important differences between the submitted version and the official published version of record.

People interested in the research are advised to contact the author for the final version of the publication, or visit the DOI to the publisher's website.

- The final author version and the galley proof are versions of the publication after peer review.

- The final published version features the final layout of the paper including the volume, issue and page numbers.

Link to publication

\footnotetext{
General rights rights.

- You may freely distribute the URL identifying the publication in the public portal. please follow below link for the End User Agreement:

www.umlib.nl/taverne-license

Take down policy

If you believe that this document breaches copyright please contact us at:

repository@maastrichtuniversity.nl

providing details and we will investigate your claim.
}

Copyright and moral rights for the publications made accessible in the public portal are retained by the authors and/or other copyright owners and it is a condition of accessing publications that users recognise and abide by the legal requirements associated with these

- Users may download and print one copy of any publication from the public portal for the purpose of private study or research.

- You may not further distribute the material or use it for any profit-making activity or commercial gain

If the publication is distributed under the terms of Article $25 \mathrm{fa}$ of the Dutch Copyright Act, indicated by the "Taverne" license above, 
Appraisal of self-care agency

A.S.A.-scale 



\section{APPRAISAL OF SELF-CARE AGENCY A.S.A.-SCALE}

Reliability and Validity testing of the Dutch version of the A.S.A.-scale measuring Orem's concept 'Self-Care Agency'

\section{Proefschrift}

ter verkrijging van de graad van doctor aan de Rijksuniversiteit Limburg te Maastricht, op gezag van de Rector Magnificus, Prof.Dr. F.I.M. Bonke, volgens het besluit van het College van Dekanen, in het openbaar te verdedigen op donderdag, 29 juni 1989 om 16.00 uur

door

Georges Christoffel Maria Evers geboren te Valkenswaard in 1950

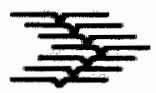

Van Gorcum, Assen/Maastricht, 1989 


\section{Promotores:}

Prof.dr. M.A. Isenberg, Wayne State University, USA

Prof.dr. H. Philipsen

Beoordelingscommissie:

Prof.dr. F. ten Hoor, voorzitter

Prof.dr. I. Abraham, University of Virginia, USA

Prof.dr. A.H.M. van den Bergh-Braam

Prof.dr. J.A. Knottnerus

Prof.dr. G.J. Kok

Prof.dr. M. Lorenzen, University of Oslo, Norway 


\section{Contents}

Acknowledgements ................................................................... VII

Introduction ....................................................................... IX

1. Purpose of the Study ............................................................ 1

1.1 Need for Theory Testing in Nursing ......................................... 1

1.2. Orem's Self-Care Model as paradigm for theory testing ................ 4

1.3. Analysis of Self-Care Agency measurement instruments .............. 12

1.4. Conclusion ............................................................................ 16

2. Development of the A.S.A.-scale ............................................ 19

2.1. Literature Review .............................................................. 19

2.2. Scale Construction .............................................................. 26

2.3. Content Validation ........................................................... 29

2.4 Translation ....................................................................... 30

3. Hypotheses …....................................................................... 32

3.1. Reliability and Validity of Measurements................................. 32

3.2. Internal Consistency ............................................................. 35

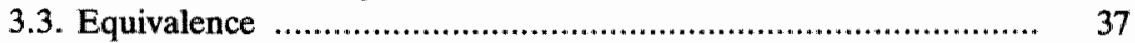

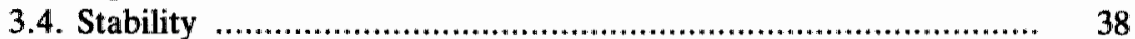

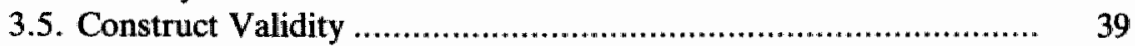

3.6. Measurement Conditions ..................................................... 46

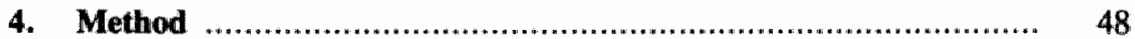

4.1. Integrated Research Program .................................................. 49

4.2. Study I: Difference in Self-Care Agency within an Elderly Population (ELPO) ..................................................................... 51

4.3. Study II: Self-Care Agency, A.D.L. and Social Support of Rheumatoid Arthritis Patients at Home (RAP)

4.4. Study III: Self-Care Agency of Institutionalized Rehabilitation Patients measured by E.S.C.A. and A.S.A. (REHAB) .................. 58

4.5. Study IV: Appraisal of Acute Care Hospital Patients' Self-Care Agency by Nurses, Patients and Significant Others (HOP) ............

4.6. Study V: Self-Care Agency and A.D.I. of Patients discharged from an Acute Care Hospital (DIHOP) 
4.7. Study VI: Change in Patients' Self-Care Argency after Coronary Artery Bypass Surgery (CABS)

4.8. Study VII: Self-Care Agency and sex-stereotyped Self Image of Healthy College Students (COLS)

4.9. Summary of hypothesis in relation to studies...

5. Results

5.1. Total A.S.A-A scores

5.2. Internal consistency ............................................................ 81

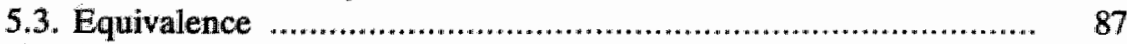

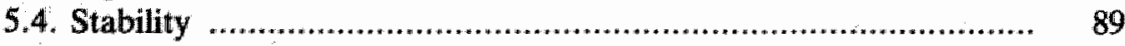

5.5. Construct Validy ................................................................. 91

5.6. Measurement Conditions ....................................................... 106

6. Discussion and Conclusions ................................................ 108

6.1. Reliability .......................................................................... 108

6.2. Construct Validity ............................................................ 113

6.3. Operational Consequenses ..................................................... 119

6.4. Limitations and Implications ............................................... 120

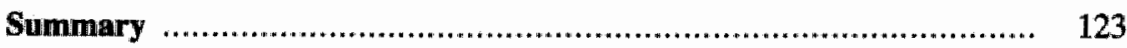

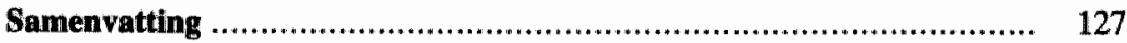

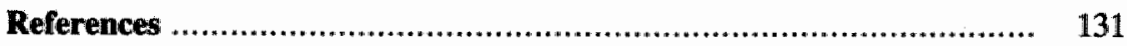

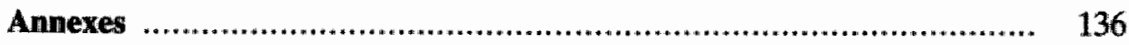

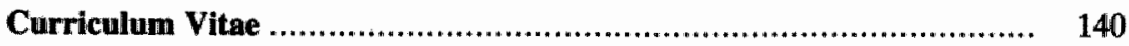




\section{ACKNOWLEDGEMENTS}

The research reported here is the result of assistance and support of many persons. They contributed to completion of this study by stimulating me and by sharing their knowledge, skill, experience and time. I realize with regret that to acknowledge a few is to neglect many more.

To my promotores Marjorie Isenberg and Hans Philipsen I express my sincere appreciation and warm regards. Both have generated and stimulated my interest in Theory based Nursing Research. Marjorie as my mentor in matters of Nursing Theory and Hans in methodological issues. Your wisdom, scolarly and personal guidance enabled me to complete this study.

To Ger Brouns and Harrie Smeets I express my thanks for their creativity, team-spirit and friendship in developing the instrument tested in this study. Ger continued to contribute substantially to instrument refinement and to support instrument testing. I also gratefully acknowledge Marina Senten. Her contribution to this study has been a substantial one both in refinement of the instrument as well as in carrying out the research project with the coronary artery bypass surgery patients. Acknowledgement deserve also two other members of the 'Maastricht Orem Research Team'. Truus Custers for her skill and persistence in creating the master-file and for the time-consuming job of dataentry. Theo van Achterberg for his important contribution to accurate and skillful data-analysis. I also wish to gratefully acknowledge him and Chantal Boderie, Rosalie Boshouwers, Annemie Courtens, Liedy Kalter, Lidwien Sijstermans, Miriam Schiltmans and Veron Schrijnemaekers for their contribution in data-collection. These graduates of the Nursing Science Program of the University of Limburg were willing to collect data for this study within their master's thesis research projects. Ruud Halfens was most helpful as consulltant in statistical analysis. I also acknowledge Marianne Tilly-Linden who assisted in data-collection as well as in preparation of figures and tables. Hanny Prick deserves acknowledgement for accurately and timely processing the text of this publication. To all my collegues of the department of Nursing Science, University of Limburg, I am deeply grateful for the time and the opportunity they gave me to complete the writing of this text. Their already heavy workload again increased substantially by this generous offer.

This research has been made financially possible by a grant of the Dutch 
Heart Foundation. This publication was sponsored by Initierend Centrum Verpleegkundig Studie Programma (I.C.V.S.).

A last and very special acknowledgement goes to José, Irene and Pauline. By constantly reminding me of my own poor Self-Care practice, they produced a healthy climate of relativism. And thanks to their care I have survived. 


\section{INTRODUCTION}

There is no quick, easy road to building the nursing science needed for informed nursing practice. What is required is a 'good' idea, courage to follow that idea and then simply the discipline to methodologically refine and test the idea (Walker, 1986). This probably is an accurate description of the scientific endeavour reported in this study.

Lack of reliable and valid instruments to measure nursing concepts and their interrelations seems to hinder the advancement of nursing as a scientific health care discipline. The aim of this research project was to contribute to nursing's development as one of the Health Sciences. This study's contribution is limited to reliability and validity testing of an instrument developed to measure a concept of a nursing theory. As a consequence the research reported is highly instrumental in nature.

Chapter one explores the need for theory testing in nursing. Orem's SelfCare Deficit Theory in generall and the concept of Self-Care Agency in particular are identified as the focus of this study. Reliability and validity testing of an instrument developed to measure this concept is stated as the purpose of this study.

Development of the Appraisal of Self-Care Agency A.S.A.-scale is described in chapter two. Included are a review of the literature and a description of scalle construction. Both content validation and translation of the English version into Dutch are reported.

The concepts of reliability and validity are defined in chapter three. Based on these defintions hypotheses are stated in relation to internal consistency, equivalence and stability of the A.S.A.-scale. Hypotheses related to construct validity are mainly derived from Orem's Self-Care Deficit Theory for nursing. Included are also some hypotheses related to measurement conditions.

Chapter four contains the method's section. An integrated research program was developed to test the stated hypotheses. Seven studies are reported, each contribating its piece to the total progran of research to test for reliability and validity of the scale. Included are studies with elderly, with reumatoid arthritic patients, rehabilitation and coronary artery bypass surgery patients; with patients staying in and discharged from acute care hospitals; and with young healthy college students.

Chapter five reports results. Total A.S.A.-scores are described and com- 
pared for the different studies. Results are reported for hypotheses stated to test for internal consistency, equivalence, stability and construct validity as well as for hypotheses related to measurement conditions.

In the last chapter results are discussed in relation to reliability and validity of the A.S.A.-scale. Methodological limitations of the study are also discussed. The chapter concludes with theoretical as well as practical implications of this study. 


\section{PURPOSE OF THE STUDY}

\section{Introduction}

This chapter explores the need for theory testing in studying client problems, nursing interventions and their influencing conditions. Given the pre-paradigmatic stage in the development of nursing science one out of several competing theoretical perspectives had to be taken. Both theoretical and practical reasons are discussed for choosing Orem's Self-Care Deficit Theory for Nursing. SelfCare Agency can be considered as one of the core concepts of this theory. Instruments developed to measure this concept are analysed. As a consequence reliability and validity testing of a new instrument emerges as the purpose of this study.

\subsection{Need for Theory Testing in Nursing}

\section{Knowledge as a basis for practice}

Nursing is practical rather than theoretical in nature. This does not relieve nursing of the necessity for basing its practices upon scientific concepts (Gunter, 1962). The need for knowledge which is specific to nursing has been recognized since the beginning of modern nursing. Florence Nightingale wrote in 1859:

'I believe .... that the very elements of nursing are all but unknown ..... are as little understood for the well as for the sick. The same laws of health or of nursing, for they are in reality the same, obtain among the well as among the sick'. (Nightingale, 1859, p.6).

The very elements which Nightingale identified focused on health, on the environment and on nursing interventions such as nourishment and observation of the patient and on the interpersonal relationship between nurse and patient (Newman, 1972). According to Nightingalle these elements of nursing were all but unknown and little understood. In general knowledge is gained by research and in a field such as nursing to some degree by practice. Understanding is gained by theory. A science develops through progress on both of these fronts (Walker, 1986). 
From the first decades of this century on the number of studies in nursing done by nurses and non-nurses has rapidly increased. A major step in directing the attention of nurses in North America to research was accomplished with the publication of the first issue of Nursing Research in 1952. The value of the publicated studies as contributing to a science of nursing was debated from 1962. Many studies seemed not to have been done within an explicit theoretical system designed to discover either facts or relationships between facts which were applicable to nursing (Gunter, 1962). Throughout most of this century the approach to nursing was based largely on medical knowledge and to some extend this focus still persists (Newman, 1972). Another approach to nursing knowledge has been pursuit of the educational process and method. The earliest opportunities for nurses to pursue graduate education were provided by schools of education. Consequently the research of these nurses was primarily related to educational and to some extend administrative problems in nursing. The large proportion of non-nurse contributions during the early periods may also explain the bulk of studies on functions and characteristics of nurses (Newman, 1972). During those years nurses in North America tended to turn to social scientists for help in studying nursing. This approach resulted in restatement of nursing problems as social science questions with nurses studying nurses rather than client problems and nursing interventions (Wald and Leonard, 1964). In later years nursing's proper phenomenon of concern has been identified and described as 'diagnosis and treatment of human responses to health problems' (ANA, 1980). In an analogous way this proper phenomenon of concern to nursing has been described by the Dutch National Council for Health Affairs (N.R.V., 1984, 1988). Thus relevant knowledge as a basis for nursing practice is generated by research questions dealing with client's problems in relation to nursing interventions.

\section{Need for relewant theorizing}

Knowledge is gained by research, understanding by theory. Concern about nursing theory has received considerable attention since 1968. During this period three main approaches to the discovery of nursing theory emerged (Newman, 1972). The first approach was the borrowing of theories from other disciplines with an intent to integrate them into a science of nursing. The second was the analysis of practice situations in search of the theoretical underpinnings and the third was the creation of conceptual models or paradigms from which theories could be derived.

Wald and Leonard (1964) can be cited as early opponents of the first approach of borrowing theories from other disciplines. They criticize the attempt 
to make borrowed concepts fit and seriously doubt that the integration of relevant basic sciences into clinical nursing is a viable means of building nursing theory given the present state of the behavioral sciences and nursing. More recently Walker (1986) has stated that although many theories from other disciplines are relevant to nursing the testing of these theories within a framework of for instance physiology, sociology or psychology relate the data more clearly to these disciplines than to nursing.

Walker (1986) argues against the second or inductive approach to develop theory on the basis of practical nursing experiences as the proposed method of discovery by citing Hempel (1966). 'There are .... no generally applicable rules for induction by which theories and hypotheses can be mechanically derived or inferred from empirical data .... Scientific hypotheses and theories are not derived from observed facts but invented'. Wallker summarizes the logical difficulties of induction as: the failure to account for the selecting principles that render data to categories of data; and the failure to account for the move from merely observing data to putting forth conceptualizations that organize data. In reaction to the logical rejection of induction the notion of retroduction has been introduced by Hanson (1958). He described this iterative process as using both induction and deduction sequentially to arrive at adequate theoretical formulations. This notion postulates that in attempting to conceptualize a given set of events the inquirer uses a certain point of view.

As the third main approach to the discovery of nursing theory the creation of conceptual systems or models emerged. Walker and Avant (1983) call them grand nursing theories, which have provided global perspectives for nursing practice, education and research. Fawcett and Downs (1986) defined a conceptual model as a set of abstract and general concepts and propositions that provides a distinctive frame of reference for the phenomena of interest to a discipline. A conceptual model is synonymous with a paradigm or a disciplinary matrix. It reflects the philosophical stance, cognitive orientation and research tradition of a group of scholars rather than the beliefs, values, thoughts and research methods of all members of a discipline. Each conceptual model provides a focus which directs the questions one askes and the theories one proposes and subsequently tests. It provides a network within which questions, theories and data fit together (Newman, 1979).

Comparison of these different approaches leads to the conclusion that an iterative process of induction and deduction is needed. Furthermore a distinctive frame of reference (paradigm) is essential for relevant theorizing in nursing.

\section{Need for empirical support}

Kuhn (1970) points out that the early stage of scientific development, the 
pre-paradigm stage, is characterized by divergent schools of thought which although addressing the same range of phenomena usually describe and interprete these phemomena in different ways. Nursing appears to be now in this pre-paradigm stage (Hardy, 1978). In discussing the advancement of scientific knowledge Kuhn (1970) suggests that paradigms are endorsed by a discipline and its subgroups because of their scientific empirical support. The existence of a prevailing paradigm facilitates the normal work of science. Research is purposeful, orderly and raises few unanswerable questions. When a dominant paradigm doesn't exist, a discipline can be classified in a pre-paradigmatic stage. Such is the status of nursing today, with energy going into attempts to justify one of the several paradigms rather than into purposeful, orderly research. Nursing research that is conducted is often poorly focused and unsystematic (Hardy, 1978). Until there is a prevailing paradigm and some exemplar paradigms to give focus to the thinking and work of nurse-scientists knowledge in nursing will develop sllowly and somewhat haphazardly. This leaves the practicing nurse in a difficult position of deciding what knowledge is usable and how it should be evaluated for use.

If paradigms are to prevail in a discipline they must attract an enduring group of adherents and there must be generated sufficient scientific-empirical support. As a consequence theory testing is a vital next phase which needs to follow the initial development of theory. In building nursing science reciprocal links are necessary between the context of discovery and justification. Unfortunately the struggle to develop theory in nursing until now has often left the testing of theory neglected (Walker, Avant, 1983). But as Marx (1963) so accurately has stated we need to recognize most explicitly that both discovery and confirmation are necessary to effective scientific work. The most ingenious theories are of limited value until empirical tests are produced; the best confirmed proposition is of little value unless it deals with meaningful variables.

\subsection{Orem's Self-Care Model as paradigm for theory testing}

\section{Major components of a nursing paradigm}

The domain of nursing as field of knowledge has always included the nurse, the patient, the situation in which they find themselves and the purpose of their being together, the health of the patient (Newman, 1983). In more formalized terms the major components of a nursing paradigm are: Nursing (as an action); Client (human being); Environment of the client, the nurse and of the nurseclient; and Health. The nurse interacts with the client and the environment for the purpose of facilitating the health of the client.

Within the context of these four major components and their interrelationships conceptual models can be examined (Newman, 1983). 


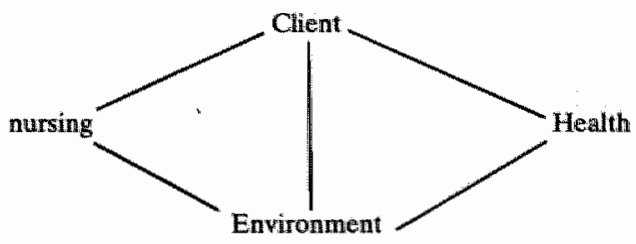

(Source: Newman, M.A., in: Chaska, N.L., 1983, p.388).

The first major emphasis in the development of nursing theory based on Nightingale"s work (1859) was given to environment. This emphasis on environmental factors continued well into the twentieth century. A shift occured in the 1950 's to the nature and processes of the nurse-client relationship influenced by the works of Peplau (1952), Orlando (1961) and later continued by King (1971, 1981). At the same time other theorists emphasized the purpose of nursing and the nature of nursing practice in explicitly trying to differentiate nursing from medical practice. Orem described nursing in terms of assistance in respect to needs for self care (Orem 1956, 1959, 1971, 1980, 1985), Henderson (1955) as doing for the patient what he cannot do for himself, Johnson (1961) defined equilibrium, or dynamic stability as the goal of nursing care. Roy $(1970,1981$, 1984) stated nursing's role as facilitating the adaptive potential of the patient. Roger's (1970) conceptual model provided a fourth shift and emphasized the unitary nature of man and the inseparability of man and environment in understanding the life-process of man. Currently there is increasing emphasis on the concept of health (Newman, 1979, 1986). Another focus is that of cultural diversity and universality of care-practices as the essence of nursing (Leininger, 1978, 1980, 1984, 1.985).

\section{Orem's Self-Care Model chosen as a Nursing Paradigm}

Orem"s Self-Care Model has been chosen as the frame of reference for this study (Orem, 1956, 1959, 1971, 1980, 1985). It has been stated at the beginning of this chapter that nursing is rather practical than theoretical in nature. In practice disciplines, such as nursing it seems preferable to seek and organize knowledge for the sake of the work to be done. Orem's Self-Care paradigm offers the opportunity to describe and explain nursing practice. In theorizing Orem explicity differentiates the nature of nursing practice from that of medical practice. According to Orem every human service has a special concern for some aspect of human functioning that defines what the service differentiates from other services. Men, women and children are the object of all direct 
human care services of physicians, clinical psychologists, nurses, social workers etc. The reason why individuals require nursing is referred to by Orem as their need for self-care.

Orem further states that modern society expects adults to be self reliant, responsible for themselves and for the well being of their dependents. Most social groups further accept that persons who are helpless, sick, aged, handicapped or otherwise deprived should be helped in their immediate distress. Thus both self-help and help to others are valued by society as desirable activities. Nursing as a specific type of human service is based on both values. As such Orem's paradigm is congruent with the social movement for self-care as described by Levin, Katz and Holz (1976). Inadequate, expensive health care services for the increasing number of chronically ill are considered the major force in the social movement for an increased role of lay resources or persons in health care delivery.

One of the major difficulties in the Self-Care literature in general is the lack of a commonly accepted definition of Self-Care across disciplines (Levin, Katz and Holz, 1976; Brugge, 1981). Different conceptualisations of Self-Care are based upon differences in who initiates the health-care activities, who is in control of the professional-lay interaction and the scope of the behaviours, activities (Brugge, 1981). In this respect it is important to realize that Orem's concept of Self-Care is substantially different from Levin's definition of SelfCare (Levin e.a., 1976; Levin, 1977) from Katz Independent Performance of Activities of Daily Living ADL (Katz e.a., 1963; Katz, 1976, 1983) and the idea of Self-Help.

In the Netherlands Hattinga Verschuere (1977) is one of the best known representatives of the Self-Help social movement model. This model as well as Orem's conceptual idea's have also strongly influenced Dutch publications in the field of nursing (van den Brink Tjebbes, 1979, 1982; Evers e.a., 1981; de Jong, 1983; van der Peet, 1983, 1984, 1985). Analysis of the most well known Dutch nursing model of Self-Care (van den Brink Tjebbes, 1979, 1982) led to the conclusion that there is insufficient semantic clarity and logical consistency in the model and that formalized propositions are lacking (Brouns, Evers, 1982). As a consequence Orem's Self-Care paradigm was given preference for theory testing in this study.

In reading the following pages and chapters, especially two implications of the choice should be taken into account. Firstly Self-Care and Self-Care Agency are not restricted to primary activities by the person him or herself. Seeking adequate professional help can be excellent Self-Care and Self-Care Agency in Orem's vision. They are not to be equated with Self-Help as a social obligation. Secondly Self-Care and Self-Care Agency are logically independent from the 
execution of physical activities. Persons who are not able to bath themselves or have to use a wheelchair can have a high degree of Self-Care and Self-Care Agency. They are not to be equated with a lack of restriction in Daily Life Activities.

In 1956 Dorothea Orem characterized nursing as: 'specialized assistance to persons with disabilities of such a character that more than ordinary assistance is necessary to meet daily needs for self care and to intelligently participate in the medical care they are receiving from the physician'. In 1959 she describedt the condition that validates the requirement for nursing as "the inability of a person to provide continuously for self the amount and quality of required self-care because of the situation of personal health'. She conceptualized SelfCare as the personal care that human beings require each day. This expression of nursing's proper object conforms in substance with that in Virginia Hendersons's 1955 statement about nursing (Orem, 1985, p.19). Self-Care is described as a requirement for every person. Self-Care action is needed in order to sustain life and health, recover from disease or injury and cope with their effects (Orem, 1985, p.34). The amount and quality should be 'therapeutic'. The word 'therapeutic' in this context refers to supportive of life processes, maintenance of normal growth, development and maturation; prevention, control or cure of disease processes and injuries; prevention of or compensation for disability and promotion of well being (Orem, 1985, p.89). In the term Self-Care the word self is used in the sense of one's whole being. Self-Care carries the dual connotation of 'for one-self' and 'given by one-self'. Orem (1985, p.88) refers to it as deliberate action that is practical in orientation. Performing Self-Care involves a decision, a choice. This is so even with routine practice such as related to food selection or personal hygiene. Unless Self-Care activities have become habitual practices, there is a need for reflection about what should be done and how it will be done.

\section{Major concepts}

The first concepts formulated by Orem in 1959 focused on the condition that gives rise to requirements for nursing and the product of nursing. Between 1960 and 1980 Orem working alone and with collegues in the Nursing Development Conference Group $(1973,1979)$ laid out the broad conceptual structure of nursing. The work of this phase culminated in the expression of Orem's General Theory of Nursing and the explication of three theories: the Theory of SelfCare Deficit, the Theory of Self-Care and the Theory of Nursing Systems. These theories appear in Orem's 1980 and 1985 editions of 'Nursing: concepts of practice'.

The conceptual model of the General Theory of Nursing is comprised of six interrelated core concepts: 'Self-Care", 'Self-Care Agency', "Therapeutic SelfCare Demand', 'Self-Care Deficit", 'Nursing Agency' and 'Nursing Systems'. 
The four first concepts are related to persons in need of nursing; 'Nursing Agency" is oriented to the nurse and "Nursing Systems" to the actions of nurses.

Self-Care 'Self-Care is the practice of activities that individuals initiate and perform on their own behalf in maintaining life, health and well-being' (Orem, 1985, p.84). It is a particular kind of deliberate action that persons engage in to regulate their own functioning and development. Self-care practices are directed to the self or to conditions in the environment. They are learned behaviors that are culturally or scientifically derived.

Self-Care Agency 'Self-Care Agency is the complex acquired ability to meet one's continuing requirements for care that regulates life processes, maintains or promotes integrity of human structure and functioning and human development, and promotes well-being' (Orem, 1985, p.105). It is the ability of the individual to engage in the operations required for Self-Care. In estimative operations the person investigates the situation and determines what actions should be taken. In productive operations the individual performs the actions necessary to bringing about a change or to maintain a certain state.

Therapeutic Self-Care Demand "The measures of care required at moments in time in order to meet existent requisites for regulatory action to maintain life, to maintain and promote health, development and general well-being' (Orem, 1985, p.31). It is the collective term for the totality of actions to be taken or work to be done in order to meet Self-Care Requisites. Orem describes SelfCare Requisites as the purposes to be attained through the kind of actions termed Self-Care (Orem, 1985, p.85). She devides Self-Care Requisites into three types: Universal, Developmental and Health Deviation. The Universal Requisites are common to all human beings and are associated with life processes and maintenance of human integrity. Developmental Requisites are associated with processes and events that occur during a particular phase in the life cycle. Health Deviation Requisites are associated with genetic defects, illness, disease, injury or other abnormal variations in human functioning. Orem identifies eight Universal Self-Care Requisites (Orem, 1985, p.90, 91): the maintenance of sufficient intake of air, of water and of food. The provision of care related to elimination and excretements. The maintenance of balance between activity and rest and between solitude and social interaction. The prevention of hazards to human life, functioning and well-being and the promotion of human normalcy. The eight Universal Self-Care Requisites are interrelated. They vary in nature and quantity from one individual to another. The need for an individual to take action in regard to a requisite may manifest itself in a variety of ways. Individuals may or may not be able to calculate their own Therapeutic Self-Care Demands.

Self-Care Deficit 'Self-Care Deficit is a relationship between Self-Care Agen- 
cy and Therapeutic Self-Care Demand in which Self-Care Agency is not adequate to meet the known Therapeutic Self-Care Demand" (Orem, 1985, p.31). Self-Care Deficit is a function of Self-Care Agency and Therapeutic Self-Care Demand. Self-Care Deficits are associated not only with limitations for performing care measures but also with the effectiveness of Self-Care. Self-Care Deficits can be described as partial or complete and as existent or as emerging.

Nursing Agency "Nursing Agency is the complex capability for action that is activated by nurses in their determination of needs for, design of and production of nursing for persons with a range of types of Self-Care Deficits' (Orem, 1985, p.31). It is the specialized ability acquired through educational preparation and experience to provide assistance to other individuals in meeting Self-Care Demands. Methods of assisting may consist of one or all of the following: acting or doing for, teaching, guiding, supporting and providing a developmental environment. The Nursing Agency of individual nurses varies with their educational preparation; their perspective of nursing practice; their mastery of the technologies of nursing practice and their abilities to care for others through nursing practice.

Nursing System 'Nursing System is a continuing series of actions produced when nurses link one way or a number of ways of helping to their own actions or the actions of persons under care that are directed to meet these persons's 'Therapeutic Self-Care Demands or to regulate their Self-Care Agency' (Orem, 1985, p.31). Nursing Systems are action systems through which nurses appraise the ability of individuals to effectively engage in Self-Care; enhance the development and operability of persons's Self-Care Agency and ensure the effective meeting of existing Self-Care Demands. Orem describes three basic designs of Nursing Systems: Wholly Compensatory, Partly Compensatory and Supportive-Educative. The design of each system clearly expresses the scope of nursing responsibility, the roles of nurse and patient and the kinds of actions to be performed by the nurse and patient in regulating the patient's Self-Care Agency and in meeting the patient's Therapeutic Self-Care Demand.

\section{Interrelations and Propositions}

The six major constructs interrelate to provide focus and boundaries for nursing. They are theoretical concepts that describe properties, conditions and actions of persons in nursing practice situations. Figure two represents five of the concepts in their interrelationships, Nursing System is not shown in the the figure.

Self-Care provides the basis for the model and thus partially defines each of the other constructs. Therapeutic Self-Care Demand activates Self-Care Agency and Self-Care Requisites provide purpose for Self-Care Agency. When Self- 


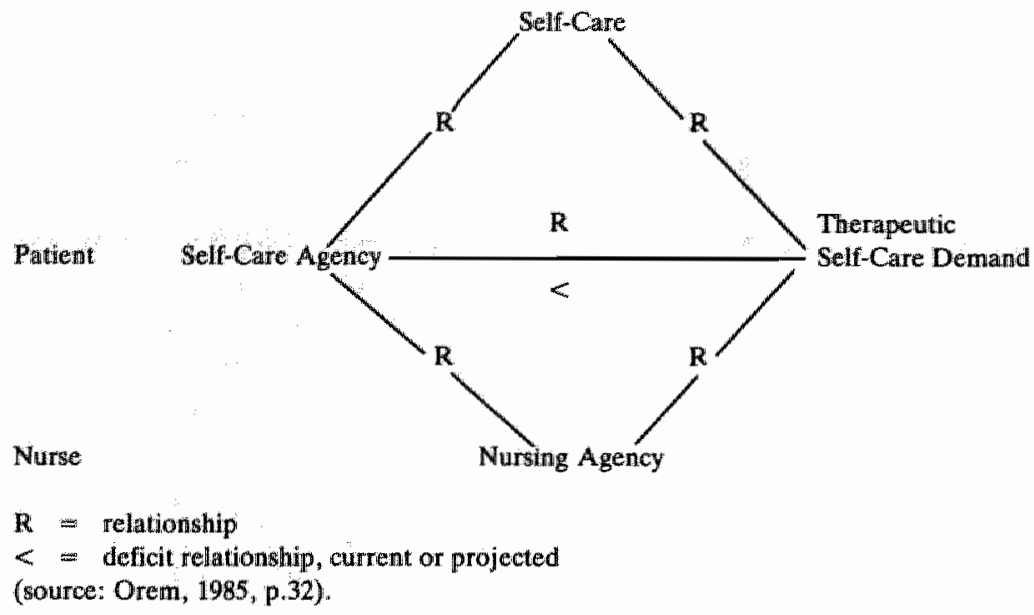

Care Agency is inadequate to meet the Therapeutic Self-Care Demand a Self-Care Deficit exists. A Self-Care Deficit signals the need to activate Nursing Agency. Nursing Agency assists with the achievement of Self-Care by meeting Therapeutic Self-Care Demands and increasing Self-Care Agency. When SelfCare Agency is sufficient to meet Self-Care Demands Nursing Agency is no longer required by the individual. Basic conditioning factors may change the Self-Care Requisites or influence the value of Self-Care Agency.

The relations between the theoretical concepts are made explicit in the propositions of three related theories: a Theory of Self-Care Deficit; a Theory of Self-Care and a Theory of Nursing System. They form the constituent parts of a General Theory of Nursing (Orem, 1985, p.34).

The Self-Care Deficit theory denotes the proper human object or focus of nursing in social groups. It provides criterion measures for identifying those who need nursing. It explains when and why nursing is required.

The Theory of Self-Care explains Self-Care as a human regulatory function and denotes the relationship between Self-Care practices and functional integrity and human functioning and human development. It describes and explains why these forms of care are necessary for the continuance of life.

The Theory of Nursing System(s) explains the relationship of the product of nursing to the reason why people need and can benefit from nursing. The theory describes the product of nursing as a Nursing System through which the capability of persons to engage in Self-Care is regulated and Self-Care Demands are known and met. It explains how people can be helped through nursing. 
The Self-Care Deficit Theory is the core of the General Theory of Nursing. Self-Care Agency can be considered as a core concept of the Self-Care Deficit Theory. As such it has been choosen as the focus of this study.

\section{Use of Orem's Theories in research}

Fawcett and Downs (1986) have proposed the following criteria for analysing the use of nursing theories in research. Criteria include the significance, internal consistency and testability of the theory.

A significant theory should reflect the phenomena of interest to a discipline. Orem"s General Theory of Nursing encompasses considerably well-refined descriptions of mursing as an action, client as a human being, environment and health (Fawcett, 1984). The criterion of significance requires also that a theory provides explanatory power. The Self-Care Deficit Theory explains when and why nursing is required. The Theory of Self-Care explains why this form of care is necessary for the continuance of life. The Theory of Nursing Systems explains how people can be helped through nursing. These theories can be applied to practice situations with a variety of individuals involved, at different times and different places. Thus they can be classified as broad in scope with high explanatory value. A significant theory also generates considerable attention from the relevant scientific community as it provides a new insight into a phenomenon or a new way of viewing a phenomenon. Orem's Self-Care model has influenced the thinking and work of many nurses in North America and elsewhere (Fawcett, 1984).

Semantic clarity and consistency of a theory is a second criterion for analysing the use of Nursing Theories in research. During twenty years Orem worked alone and with collegues of the Nursing Development Conference Group on concept formalization (Orem, 1971, 1973, 1979, 1980, 1985). This has resulted in a framework of six clearly defined concepts and three formal theories. The propositions of the Self-Care Deficit Theory reflect structural consistency (Fawcett, 1984). Orem's theories further clearly reflect the organismic world view without evidence of a mechanistic view. Orem also translates two potentially competing views of persistence and change by emphasizing that Self-Care Agency is conditioned by age and developmental state. As such they do not present a logical inconsistency (Fawcett, 1984).

Testability frequently is regarded as the primary characteristic of a scientifically useful theory in a practice discipline such as nursing. A theory is testable if concepts can be measured and if its derived hypotheses can be falsified (Fawcett, Downs, 1986). Backscheider (1974) has reported a systematic attempt to assess Self-Care Requisites and Self-Care capabilities of outpatients with diabetes mellitus. No formal controlled study based on this work was reported. Horn and Swain (1977) reported the development of an instrument to measure health 
status of patients by identifying ability to perform Self-Care as one of the domains of health status of patients. Self-Care of hospitalized adult schizophrenics has been studied by Underwood (1978). Self-Care Behavior among elderly residents of public housing was studied by Evans (1979). She analysed the relationship of need awareness, locus of control, health state, social support system and social interaction of these residents. Dodd (1980) reported a study of Self-Care of cancerpatients who are receiving chemotherapy. The relationship between family-coping strategies and Self-Care during cancer chemotherapy treatments was studied by Musci (1983). Self-Care was also studied in relation to patient's cognitive structure (Neves, 1980) and stages of ego development (Gast, 1983). Fernsler (1983) compared patients' and nurses perceptions of patients Self-Care Deficits associated with cancer chemotherapy. Measurement of Self-Care Agency was reported for adult college students by Kearney and Fleischer (1979), for adolescents (Denyes, 1980), for school aged children (Stullenbarger, 1984), for older chronic patients (Hungelmann, 1984). Recently Hanson and Bickel (1985) reported the development of an instrument to measure perceptions of Self-Care Agency. Self-Care Agency was also studied in relation to health status and social support (Brugge, 1981). In conclusion Orem's theories have been tested in a number of research projects.

\subsection{Analysis of Self-Care Agency measurement instruments}

In reviewing the literature related to Self-Care Requisites Fernsler (1983) pointed out as a major limitation of the reviewed studies the failure to establish validity and reliability of the tools. There seems to be an equal need to assess validity and reliability of instruments used to measure concepts of Orem's theories in general and in particular the concept of Self-Care Agency being the focus of this study.

\section{Exercise of Self-Care Agency ESCA-scale}

The Exercise of Self-Care Agency Scale ESCAS (Kearney and Fleischer, 1979) is a 43 item self-appraisal instrument. Each item on the scale is rated along a five-point Likert scale ranging from very characteristic, somewhat characteristic, no opinion, somewhat uncharacteristic to very uncharacteristic. Of the total scale 29 items are positively oriented towards Exercise of Self-Care Agency and 14 negatively. The scores go from 0 (very uncharacteristic to 4 (very characteristic) for the positive items and the reverse for the negative items. The theoretical minimum score is 0 and the maximum score is 172 , indicating a high degree of Exercise of Self-Care Agency.

Kearney and Fleischer viewed the construct "Exercise of Self-Care Agency" as a measurable dispositional trait of a person. To support this stance they refered to the 1973 publication of the Nursing Development Conference Group. There it is stated that Self-Care conduct is ego-processed, learned 
through interpersonal relations and communication, affected by self concept and by the level of maturity of the individual. Kearney and Fleischer stated that at the time of instrument development there was no clearly accepted operational definition of Exercise of Self-Care Agency. Consequently they used the inductive approach to identify components of Exercise of Self-Care Agency and to provide a conceptual framework for generating questionnaire items. Concept clarification was done by a group of four graduate student-nurses and a psychologist. By concensus the group delineated four subconstructs that contribute to a person's Exercise of Self-Care Agency a) an active versus a passive response to situations, b) motivation, c) knowledge base, d) sense of self-worth. The following indicants for these subconstructs were identified: a) an attitude of responsibility for self, b) motivation to care for self, c) the application of knowledge to Self-Care, d) the valuing of health priorities and high self esteem. Items for each indicant were formulated. Content validity of the initial instrument was established by a panel of five experts who rated each item as to its worth as an indication of Exercise of Self-Care Agency. Of the original 44 items twenty-nine were rated as "good" with $80 \%$ interrator agreement, fifteen had $60 \%$ interrator agreement as to good or fair, one item was reworded and one was eliminated. The instrument was tested on a convenience sample of 76 nursing students ( 70 women, $6 \mathrm{men}$ ) and 153 psychology students ( 88 women and 65 men). The mean scores for the nursing students was 122.72 (range $90-154$ SD 13.7) and the mean score of the psychology students was 120.04 (range 91-151 SD 17.7). Reliability was assessed by use of a 5-week test-retest design and split-half procedures. A test-retest reliability coefficient of Pearson $r=.77$ was obtained using only the sample of 76 nursing students. Split-half reliability coefficients of Spearman Brown $r=.81$ and .77 were obtained with the nursing student sample and the sample psychology students respectively. Construct validity was assessed by comparing responses on the instrument with scores obtained on Rotter's Internal-External Locus of Control scale, and Gough and Heilbrun's Adjective Check List. As hypothesized sigmificant positive correlations were reported between ESCA scores and the following items on the

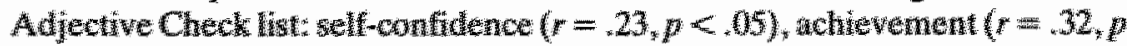
$<.01)$, intaception $(r=.26, p<.65)$, and negative correlation between

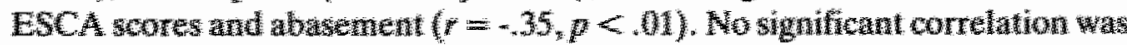
towd between ESCA and dability, nor between ESCA and interanat control as measured by Rotte" Locus of Control Scale. Though not completely consis: tent with the expectations the authors interpreted these results as consideraby supportive for construet wallidy of the instrument.

Brugge (1581) reported the we of the ESCA in a correlattonal study of the atect of heath retated dystunction and faminy as a sociat zupport system on the Exercise of Self-Care Agency in adults wh thabetes. Exercise of Sel-Care Agency was conceptwalised as the engagement in Self-Care beluvior. A con ventence sample wh wed of 51 ron-institutionalized subjects of 18 years or 
older with diabetes mellitus, of at least three month duration. The group consisted of 28 females and 23 males with a mean age of 63 years ranging from 22 to 81 . The average duration of diagnosis of diabetes mellitus was 9 years. Fifteen subjects had type I diabetes and 36 type II. Fourteen were categorized as controlled and 19 were uncontrolled. Subjects ESCAS mean score was 123 ranging from 86 to 155, SD 18.2. The coefficient alpha for ESCAS was .84. Health related dysfunction was measured with the Sickness Impact Profile, SIP (Bergner, Bobbitt, Kressel, Pollard, Gilson \& Morris, 1976). Family as social support system was measured by the Family Relationship Index, a dimension of the Family Environment Scale FES (Moos, 1974). Health related dysfunction was inversily related to Exercise of Self-Care Agency $(r=-.43, p<.05)$. Family as a social support system and Exercise of Self-Care Agency were moderately positively correlated (multiple $r=.35, p .05$ ). The cumulative ability of family as a social support system and health status to predict Exercise of Self-Care Agency was determined by multiple regression and correlation analysis. Twenty eight percent of the variance in ESCAS is explained by the cumulation of FRI and SIP (multiple $r=.53$ ) and was greater than the predictive ability of either social support $\left(r^{2}=.12\right)$ or health status $\left(r^{2}=.19\right)$ independently. One of the subscales of the Family Environment Scale was the Independence subscale. This subscalle was negligibly correlated with the ESCAS $(r=.10)$. The variables age, gender, religion, education, type, duration and control of diabetes mellitus, presence of additional chronic conditions were found to be not significantly related to Exercise of Self-Care Agency, although they could be considered as measurements of five basic conditioning factors proposed by Orem to influence Self-Care Agency. Time of the day, location of data collection, data collector and source of subject acquisition were not correlated with ESCA scores suggesting that the procedure for data collection did not influence values of ESCAS. In relation to measurement issues Brugge stated that as a measure of internal consistency a coefficient alpha of .84 is supportive of reliability of the ESCAS. Correlation coefficients of item-to-item analysis indicated that no two items

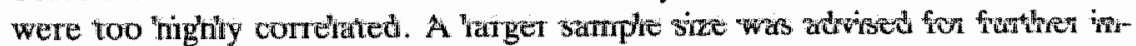
strument testing. Many subjects experienced difficulty in responding to items in the ESCAS. Kearney and Fleischer tested the ESCAS using college students. Subjects in this study were mainly elderly less educated adults. Brugge concluded that unexpected results may raise questions regarding validity of the ESCAS.

Whetstone (1987) reported the use of the ESCAS in a pilot-study in Maagdenburg, East-Germany. A convenience sample of 17 subjects was used, seven men and ten women living and working in an agricultural collective. All subjects completed the ESCAS. The instrument had been translated into German by a native born German. The results were compared with the Kearney and Fleischer study. The mean age of subjects was 46.3 years ranging from 19 to 69 . The mean score for the ESCA was $113.6,7-9$ points lower than the 
students in Kearney and Fleischer's study. The split-half coefficient for the East-German measurement was .31. The reported reliability, sample size and sampling technique make results of the study questionable.

The content domain of the ESCA scale has been analysed within the framework of the substantive knowledge of the theoretical construct of Self-Care Agency (Isenberg, 1986). Subsequent to the development of the ESCAS three major books relevant to the Self-Care Agency construct have been published: the second edition (1979) of the work of the Nursing Development Conference Group, and the second (1980) and third (1985) editions of the theoretical work of Dorothea Orem. In each of these publications the substantive domain of the Self-Care Agency construct has been further developed. Three conceptual elements of Self-Care Agency have been formulated: Human Dispositions and Capabilities foundational to Self-Care Agency, the Human Power element and the Operations of Self-Care. Using this exposition as the frame of reference each item of the ESCAS was analysed to determime which conceptual elements had been cast in the form of test items. This analysis revealed that 20 of the 43 items are expressed in terms of Human Dispositions and Capabilities identified as foundational for Self-Care Agency. Six items are reflective of the Power element of Self-Care Agency and 10 items are expressions of Self-Care Operations. Seven items were identified as irrelevant to the content domain. The instrument was found to be a global measurement of various dimensions of the Self-Care Agency construct. Clarification of what concept or concepts the scale is measuring could be served by the development of subscales. It was concluded that this instrument could not be considered as a valid measurement of Exercise of Self-Care Agency.

\section{Denyes' Self-Care Agency Instrument SCAI}

Denyes (1980) developed a self-report questionnaire consisting of 35 items to measure Self-Care Agency in adolescents. A 7-point Likert scale and an interval form questionnaire exist. The instrument measures six components of adolescents perceptions of their Self-Care Agency: ego strength and health decision making capability, relative valuing of health, health knowledge and decision making experience, physical energy levels, feelings and attention to health. Instrument construction was based on Orem's 1971 publication, on Piaget's work on cognitive development, Kohlberg's work on affective development, Tanners's publications on physical gains and Erikson's work on psychosocial development. Four major components were identified as physical, cognitive, affective moral and psychosocial. Strengths and limitations in SelfCare Agency for each of the four components were specified. Two different forms were developed for reliability and validity testing. One form contained items scaled at the ratio level asking to respond in percentages. The other contained a seven point Likert type response format ranging from never to 
always. The initial instrument consisted of 70 items. The instrument was administered through public high school cllasses to a convenience sample of 161 adolescents aged 14 to 18 years. Each youth received both forms in random order at a one week interval. A total of 37 items which demonstrated good variability, relatively normal distributions and stability across alternate forms were retained. A factor analysis was computed demonstrating six factors. A total of 35 items with loadings of .40 or above were retained. Reliability testing involved the comparison of factor analyses from two random subsets of the data. Pearson product moment correlations were computed for the pair of factors which appeared to be the same in both subsets of data. Correlations for all six pairs of factors were significant (alpha .05) and ranged from .39 to .80 . Split-half reliability coefficients for the factors with 8 or more items were $r=$ $.80, p<.01$ and $r=.83, p<.01$. To establish construct validity canonical correlations were computed between Self-Care Agency factor scores and factor scores on a Self-Care practice measurement and on Health Status measurement. Both were significant $(p=.000)$. Denyes concludes her study with a comparison between the components of Self-Care Agency identified in her research and those published after her initial instrument development. Some of the components were similar some different. NDCG components number 3, 4 and 10 did not appear in the SCAI. SCAI components 'feelings' and 'valuing health' appeared to belong to dispositions foundational to Self-Care Agency. The reliability and validity estimates for this instrument were based on responses of one selected group of adolescents. According to Denyes there is no evidence that testing with other populations would yield similar results.

\subsection{Conclusion}

The need for scientific knowledge as a basis for nursing practice has been recognized from the times of Florence Nightingale. Scientific knowledge is primarily gained by research. Relevant knowledge as a basis for nursing practice is generated by research questions dealing with client's problems in relation to nursing interventions. Understanding of these phenomena is gained by theory. Theories have been borrowed from other disciplines, have been formulated on the basis of practical experiences and have been created within conceptuall models or paradigms. Comparison of these approaches leads to the conclusion that a distinctive frame of reference (paradigm) is essential for relevant theory development in nursing. Purposeful and orderly research is enhanced by the existence of prevailing paradigms. For paradigms to prevail in a discipline they need sufficient scientific empirical support. As a consequence theory testing is a vital next phase which needs to follow the initial development of theories within conceptual modells of nursing.

The major components of a nursing paradigm were identified as nursing, client, environment and health. Within the context of these components and 
their interrelationships a number of conceptual models have been developed. Orem's Self-Care model has been chosen as paradigm for this study. The model emphazises the purpose of nursing and differentiates the nature of nursing from that of medical practice. The requirement for nursing is described by Orem as the inability of a person to provide continuously for self the amount and quality of required Self-Care because of the situation of personal health. The model is comprised of six interrelated concepts: 'Self-Care', 'Self-Care Agency", 'Therapeutic Self-Care Demand', 'Self-Care Deficit', 'Nursing Agency' and 'Nursing Systems'. The relations between the concepts are made explicit in the propositions of three related theories: the Self-Care Deficit Theory, the Self-Care Theory and the Nursing Systems Theory. The Self-Care Deficit Theory is considered to be the core theory. Self-Care Agency, as a core concept of this theory, has been identified as the focus of this study. This was based on the following. Orem's Self-Care model addresses the phenomena of interest to the discipline of nursing. The Self-Care Deficit Theory provides sufficient explanatory power. The description of the Self-Care Agency concept adheres to the criterion of semantic clarity and consistency. The propositions of the Self-Care Deficit Theory reflect structural consistency. The theory has been tested in a number of research projects.

A need was identified to assess reliability and validity of instruments used in general but in particular to measure Self-Care Agency as the focus of this study. Kearney and Fleischer's Exercise of Self-Care Agency Scale (ESCAS) has been developed prior to the publication of the substantive domain of the Self-Care Agency construct by Orem and the NDCG. An inductive approach has been followed to identify components of Exercise of Self-Care Agency. Content analysis done by Isenberg resulted in questioning whether the instrument is a valid measurement of Exercise of Self-Care Agency within Orem's framework. Test-retest reliability coefficient was .77 . Split-half reliability coefficients were .81 and .77 in the Kearney and Fleischer study, .84 in Brugge's study and .31 in Whetstone's study. The latter study is questionable from a methodological point of view. Construct validity testing by Kearney and Fleischer did not lead to consistent results in terms of expected relationships with Internal Locus of Control scores. Based on unexpected results in relation to construct validity testing Brugge in her study raised questions regarding validity of ESCAS. All samples used were convenience samples of adults who were no legitimate patients except of Brugge's study that used non-institutionalized diabetics. Brugge and Whetstone's samples were relatively small. In assessing construct validity Kearney and Fleischer didn't use Orem's theoretical framework and Brugge only to a certain extent. Denyes Self-Care Agency Instrument (SCAI) was also developed prior to publication of the concept clarification done by Orem (1980) and the NDCG (1979). Comparison of the content of her instrument with the content domain developed by NDCG revealed similarities and also differences. Reliability in terms of internal consistency was reported to 
be acceptable. Reliability and validity testing was done on a convenience sample of healthy adolescents aged 14 to 18 . Denyes concluded that there is no evidence that testing with other populations would yeald similar results.

Based on the analysis of instruments to measure Self-Care Agency the following can be concluded. In respect to the instruments there is lack of sufficient conceptual clarity. Which concept or concepts of the construct SelfCare Agency, as defined by Orem, are being measured? The content validation procedure can be questioned for these early instruments. In terms of construct validity testing in most cases there is a lack of hypotheses testing based on Orem's theory. The size and choice of samples for reliability and validity testing of the instruments raise questions regarding adequacy. An obvious criterion should have been the expected discrimination in Self-Care Agency scores in populations in need for (possible) nursing care. Thus it can be concluded that there is a lack of reliable and valid instruments to measure Self-Care Agency, as defined by Orem, with adult populations in (possible) need for nursing.

As a consequence a new instrument was developed based on publications of D.E. Orem (1980) and the Nursing Development Conference Group (1973, 1979): the Appraisal of Self-Care Agency A.S.A.-scale (Isenberg M.A., Evers G.C.M., Philipsen H., Brouns G., 1989). The purpose of this particular study is:

'Reliability and Validity testing of the Dutch version of the Appraisal of Self Care Agency A.S.A.-scale'. 


\section{DEVELOPMENT OF THE ASA-SCALE}

\section{Introduction}

The Appraisal of Self-Care Agency A.S.A.-scale has been developed as a joint effort of faculty of the department of Nursing Science, University of Limburg, The Netherlands and the College of Nursing, Wayne State University, Detroit, Michigan, U.S.A. (Isenberg, M.A., Evers, G.C.M., Philipsen, H., Brouns, G., 1989). The project started July 1984 and the scale was considered testable in research projects in August 1985. The purpose of the project was stated as to create an instrument, that would be generally applicable to adults (persons of 18 years and older) in various states of health and that could be used to test Dorothea E. Orem's Theory of Self-Care Deficit.

Development of the Appraisal of Self-Care Agency (A.S.A.) scale is the subject matter of this chapter. The problem of lack of sufficient conceptual clarity was stated as one of the main conclusions of the analysis of existing instruments in chapter one. For the purpose of developing a theoretically valid measurement instrument a review of the literature was done. Based on publications of D.E. Orem (1980) and the Nursing Development Conference Group the Self-Care Agency-concept was clarified. Definition, components, characteristics and determinants were explored. This chapter further addresses the construction of the measurement instrument including conceptual demarca-

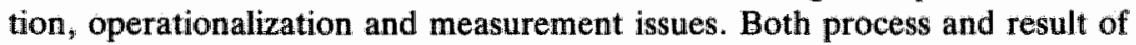
content validation are reported. The chapter ends with a description of translation procedures.

\subsection{Literature Review}

\section{Definition of Self-Care Agency}

The term 'Agency' has been attached to the concept of 'Self-Care' since the inception of the Nursing Development Conference Group (N.D.C.G.) in 1965 and has been consistently used since a N.D.C.G.-meeting in August 1969 (N.D.C.G., 1973, p.102). 'Self-Care Agency' is the term that Orem and her collegues of the N.D.C.G. use to refer to the 'power' of individuals to engage in 
Self-Care behaviors or actions (N.D.C.G., 1973, p.117; N.D.C.G., 1979, p.118; Orem, 1980, p.83). The term is a symbol for a human characteristic, a capability for a particular form of action: Self-Care. Self-Care is refered to as the actual behavior, Self-Care Agency as the capability for such behavior. Self-Care refers to actions based on culturally or scientifically derived practices which are freely performed by individuals. These practices are directed to the individuals themselves or to conditions or objects in their environments. They are performed in the interest of the individual's own life, health or well-being. (N.D.C.G., 1979, p.118). 'Agency' is used in the sense of 'Ability'. The person who exercises this power can be referred to as the Self-Care Agent or Actor (N.D.C.G., 1979, p.181). The terms Agent and Actor are used within the frame of reference of Action Theory (Parsons and Shils, 1951; Parsons, 1968; Kotarbinski, 1966; Macmurray, 1966).

The conceptual construct Self-Care Agency is utilized in two frames of reference. The first is that of individual human beings who are born into, grow, develop and function within social groups and under a range of physical, biologic and social environmental conditions. In this frame of reference SelfCare Agency is thought of as a human characteristic. The second frame of reference is that of Nursing System in which two patient variables 'Self-Care Agency' and 'Therapeutic Self-Care Demand' are conceptualised as interactive with one Nurse-variable 'Nursing Agency" (N.D.C.G., 1979, p.181).

The following propositions about Self-Care Agency summarize the positions taken by Orem and the other N.D.C.G. members from early on. (N.D.C.G., 1979, p.183). Self-Care Agency is a complex acquired human characteristic. Self-Care Agency is the power of an individual to engage in operations essential for Self-Care. The exercise of Self-Care Agency by an individual results in a) a system of actions directed to conditions in self or environment in order to regulate them, or in b) a plan for such a system of action. Self-Care Agency is an action repertoire of an individual. Self-Care Agency can be characterized as abilities and limitations for engagement in Self-Care. Conditions and factors in the environment of an individual affect development and exercise of Self-Care Agency. Individuals are subject to time sequential needs for exercise of Self Care Agency. Self-Care Agency is an estimative and a productive capability for Self-Care These propositions have given direction to further inquiry of the Self-Care Agency concept. Both the hypothetical deductive method as well as the natural history or case study method were used in this process (N.D.C.G., 1979, p.135).

\section{Distinction between Self-Care Agency and Self-Care}

One significant consideration in the development of the concept Self-Care Agency has been differentiation of the concept Self-Care from the concept 
Self-Care Agency and identification of the relationship between the two concepts. Orem and the other N.D.C.G.-members recognised early that working with these two concepts required thinking within a "person frame of reference" and not in an 'organism frame of reference' (N.D.C.G., 1979, p. 184). As such they developed the following schema locating the three concepts 'Person", 'Agency", and 'Action' in a hierarchical structure.

Figure 21. Relationship of Concepts Person, Self-Care and Self-Care Agency.

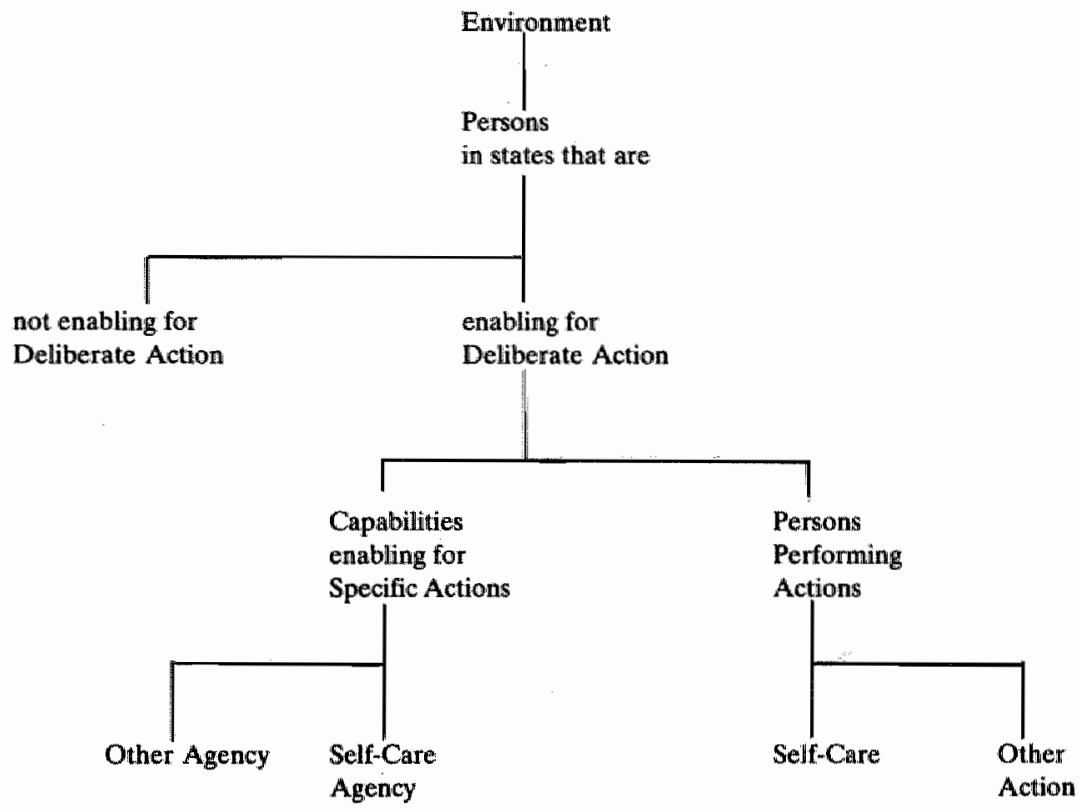

(Source: N.D.C.G. (1979). Concept Formalization in Nursing . Boston: Little, Brown and Co., p. 185).

Human Beings can be conceptualized in a range of function states. Individual Human Beings characterized as 'Persons' are devided into the logical categories of persons in states that are enabling for and not enabling for engagement in deliberate action. Persons in the former category are then devided into those with action capabilities and those performing action. This distinction brings 'Person' in relation to 'Agency' and 'Action'. Self-Care Agency is distinguished from other forms of Agency (e.g. horticultural ability) and Self-Care is distinguinshed from other forms of Action (e.g. gardening). This is based on the presupposition that action is for an end. In thinking within an agency frame nurses focus on Self-Care Agency as distinguished from other forms of agency and in the action frame as persons engaged in Self-Care as distinguished from other forms of action. 


\section{Components of Self-Care Agency}

Based on the definition of Self-Care Agency two constituent components can be identified (N.D.C.G., 1979, p.188, 189) namely: Power or capability to engage in two specified types of action, that is, Self-Care Operations of an estimative and productive type. Neither of these elements can stand in isolation since the operations make the power component a specific power. The term operation is used in the sense of action system. In estimative operations the way of acting is that of investigation, inquiry: what is to be done with respect to Self-Care. In productive operations the way of acting is regulation i.e. bringing about change in or maintaining some condition. The goal is meeting Self-Care Requisites using particular technologies. Thus the form of Self-Care Agency is conceptualized as a set of human abilities for specific deliberate action. The content of Self-Care Agency derives from its proper object: meeting Self-Care Requisites (Orem, 1980, p.83). Figure 2.2. shows the components of Self-Care Agency in their relationships to each other.

Figure 2.2. Componentis of Self-Care Agency

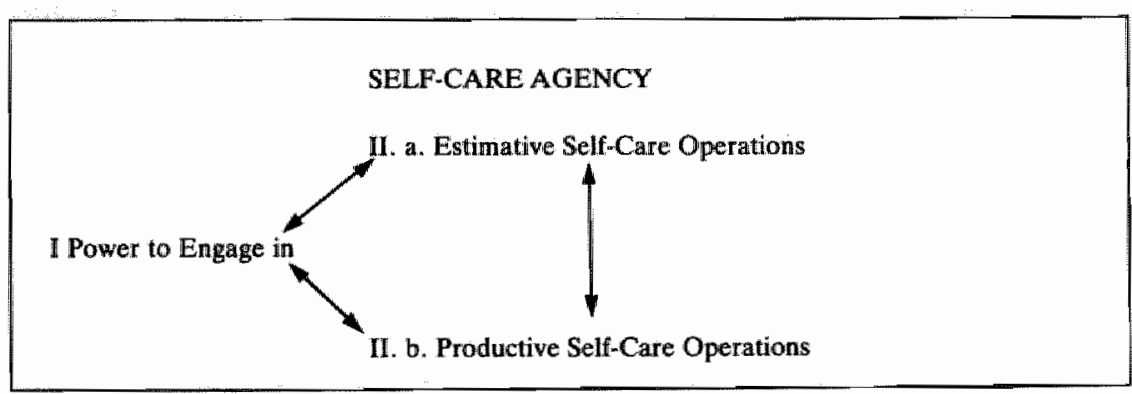

(Source: N.D.C.G. (1979). Concept Formalization in Nursing.

Boston: Little, Brown and Company, p.190).

The lines indicating relationships among the elements express that the Power element with its two qualifiers Estimative and Productive Operations is a characteristic or quality that is integral to individuals. The power that is SelfCare Agency is delimited not only by the two types of operations but also by their relationship. This is a two-way relationship since 'Estimative Operations' precede 'Productive Operations'. When the latter are started there may be a need to perform further "Estimative Operations'. The movement from investigation to regulation has been conceptualized through an additional set of operations of a transitional type. They deal with the questions: what should and what will be done. (N.D.C.G., 1979, p.189).

Ten distinct kinds of Self-Care Operations have been identified grouped in 
the afore mentioned three categories. It is assumed that these operations are related in a time sequence of before and after and that all operations are essential for Self-Care (N.D.C.G., 1979, p.192, 193)). Self-Care Operations of the Estimative type are the following. (1) Investigation of internal (self) and external conditions and factors (environment) significant for Self-Care. (2) Investigation of the meaning of these conditions and factors and their regulation for life, health and well-being. (3) Investigation of the question: how can existent conditions be regulated i.e. changed or maintained. The following two Self-Care Operations are of a Transitional type. (4) Determination which course of Self-Care should be followed and (5) deciding what to do with respect to Self-Care. The five last Self-Care Operations are of the Productive type. (6) Preparation of self, materials or environmental setting for the performance of a regulatory Self-Care action. (7) Performance of a regulatory Self-Care action within a time period. (8) Monitoring for evidence of desired or untoward effects. (9) Determination of adequacy of regulatory results and (10) decision to continue or discontinue estimative or regulatory action.

The second structure is grounded in the assumption that the Power that is Self-Care Agency is constituted from ten distinct power components each of which is associated with all or some Self-Care Operations. No associations among the power components were posited but N.D.C.G. $(1979$, p.199) recognized that associations are probable. The following Power components have been identified.

Attention and Vigilance. (I) Ability to maintain attention and exercise requisite vigilance with respect to self as Self-Care agent and significant internal and external conditions and factors.

Controlled use of Energy. (II) Controlled use of available physical energy that is sufficient for the initiation and continuation of Self-Care Operations.

Control of Body Position. (III) Ability to control the position of the body and its parts in the execution of the movements required for the initiation and completion of Self-Care Operations.

Reasoning. (IV) Ability to reason within a Self-Care frame of reference.

Motivation. (V) Motivation i.e. goal orientations for Self-Care that are in accord with its characteristics and its meaning for life, health and well-being. Decision-making. (VI) Ability to make decisions about Self-Care and to operationalize these decisions.

Knowledge-Acquisition. (VII) Ability to acquire technical knowledge about Self-Care from authoritative sources, to retain and operationalize it.

Repertoire of Skills. (VIII) A repertoire of cognitive, perceptual, manipulative communication and interpersonal skills adlapted to the performance of SelfCare

Time Ordering. (IX) Ability to order discrete Self-Care actions into relationships with prior and subsequent actions toward achievement of goals of SelfCare 
Integration. $(X)$ Ability to consistently perform Self-Care operations, integrating them with relevant aspects of personal, family and community living. These ten capabilities constituting the Power component of Self-Care Agency are considered by N.D.C.G. as formulations in need of review and testing. Their value rests in idlentification of human capabilities that serve as essential links between Self-Care operation and physiologically or psychologically described capacities of human beings in general. Thus knowledge from a number of disciplines can be articulated with the concept Self-Care Agency (N.D.C.G., 1979, p. 198).

\section{Quantitative and Qualitative Characteristics of Self-Care Agency}

The capability for Self-Care begins to develop in childhood, attains a degree of perfection in adulthood and declines with advancing age (N.D.C.G., 1979, p. 181). Self-Care Agency develops in the course of day-to-day living through the spontaneous process of learning. Its development is aided by intellectual curiosity, by instruction and supervision from others and by experience in performing Self-Care measures (Orem, 1980, p.83). Individual abilities can be described in terms of development, operability and adequacy. Development and operability of Self-Care Agency refer to quantitative characteristics, adequacy to qualitative.

Development is the characteristic expressing how much Self-Care Agency an individual has at a specific time. It is identified in terms of the kinds of Self-Care operations individuals consistently can perform (Orem 1980, p.83). Degree of development is based on the assumption that all human beings can be devided into classes of persons with undeveloped, developing and developed Self-Care Agency. State of growth and development biologically, psychologically and psychosocially explained determine the range of values of Self-Care Agency at periods throughout life span of individuals. State of growth and development is associated with chronological age, genetic and constitutional factors, sex, health state and environental conditions (N.D.C.G., 1979, p.206).

Operability is the characteristic expressing how much Self-Care Agency is in operation. It is also identified in terms of the Self-Care operations individuals consistently can perform. Given a degree of development Self-Care Agency can be fully operative, partially operative or not operative in points of time. States of physiological, psychological and psychosocial functioning are associated with levels of operability of Self-Care Agency (N.D.C.G., 1979, p.207). Operability is affected by genetic and constitutional factors as well as by culture, life experiences and health state (Orem, 1980, p.83).

Adequacy expresses the result of quality assessment of Self-Care Agency using the known Therapeutic Self-Care Demand as standard. It is measured in 
terms of the relationship of the number and kinds of operations that persons can engage in and the operations required to calculate and meet existing or projected Therapeutic Self-Care Demand (Orem, 1980, p.84). Determination of adequacy of Self-Care Agency presumes that a patient's Self-Care Requisites are known; that technologies that can be effectively used to meet them are known; and that the program of discrete Self-Care actions to be performed is known. (N.D.C.G., 1979, p.207).

There are necessary as well as possible relationships between the developmental, operative and adequacy values of Self-Care. Agency Necessary relationships exist between degree of 'development' and 'adequacy' and between degree of 'operability' and 'adequacy'. Undeveloped or developing Self-Care Agency equals not adequate Self-Care Agency. Not operative or partially operative Self-Care Agency equals not adequate Self-Care Agency. On the other hand Self-Care Agency may be developed but not operative at points in time. Developed and fully operative Self-Care Agency may be adequate or not adequate (N.D.C.G., 1979, p.209).

\section{Determinants of Self-Care Agency}

The qualitative and quantitative characteristics of Self-Care Agency are related to certain General Human Capabilities and Dispositions in an etiologic way (N.D.C.G., 1979, p.215). A survey list of relevant human capabilities and dispositions is composed of five sections. Included items name unique human capabilities or dispositions as distinguished from more comprehensive states of individuals. They can be associated with one or more of the ten abilities that constitute the power component of Self-Care Agency but not exclusively. The named capabilities and dispositions are also associated with other forms of human agency. Each capability or disposition can be examined and to some degree explained in isolation from other capabilities and dispositions. The following list of capabilities and dispositions is seen as essential for Self-Care Agency (N.D.C.G., 1979, p.212).

Basic Capabilities I. Sensation (proprioception, exteroception), learning, exercise or work, regulation of the position and movement of the body and its parts. Basic Capabilities II. Attention, perception, memory, central regulation of motivational-emotional processes.

Knowing and Doing Capabilities. Rational agency, operational knowing, learned skills (reading, counting, writing, verbal, perceptual, manual, reasoning), self-consistency in knowing and doing.

Dispositions Affecting Goals Sought. Self-understanding, self-awareness, selfimage, self-value, self-acceptance, self-concern, acceptance of bodily functions, willingness to meet needs of self, future directness.

Significant Orientative Capabilities and Dispositions. Orientations (to time, health, other persons, events, objects), priority system or value hierarchy 
(moral, economic, aesthetic, material, social). interests and concerns, habits, ability to work with body and its parts, ability to manage self and personal affairs.

Each of these five sections is viewed as reflecting a conceptualized level of human capabilities and dispositions from an action perspective. Basic Capabilities are foundational to capabilities identifed in sections 3, 4 and 5 (N.D.C.G., 1979, p.213):

The general human capabilities and dispositions are conditioned by a set of factors: Basic Conditioning Factors (N.D.C.G., 1979, p.169, p.215). Basic conditioning factor is defined as something which precipitates a chain of intrahuman events which may affect the value of Self-Care Agency. Eight factors have been identified (N.D.C.G., 1979, p.170) namely: age, sex, developmental state, health state, socio-cultural orientation, health care system (e.g. medical diagnostic and treatment modalities), family system and patterns of living. Factors contrast to Human Capabilities and Dispositions. Basic Conditioning Factors don't explain the structure of Self-Care Agency but condition its value. For example, a given magnitude of age and developmental state does not explain Self-Care Agency but is a determinant of its degree of development and therefore its value at points in time (N.D.C.G., 1979, p.171).

\subsection{Scale Construction}

\section{Conceptual Demarcation}

Two constituent components of Self-Care Agency have been identified namely Power and Self-Care Operations (N.D.C.G., 1979, p.188). Neither element can stand in isolation. Thus it was concluded that the variables of SelfCare Agency were all possible combinations of the 10 identified Self-Care Operations and the 10 Power-Components. Theoretically this resulted in 100 possibilities. As the objective of the project was to develop a measurement instrument, an extensive reduction of the variables had to take place. After several unsuccesful trials to reduce variables on a mathematical basis, it was decided to look for care elements with a high explanatory value for the SelfCare Agency-concept. The productive type of Self-Care Operations seemed adequate for this criterion. The following rationale is offered for this conclusion: (1) productive operations presuppose estimative and transitional operations, (2) productive operations are most predictive for engagement in SelfCare, and (3) possibilities to measure the productive type of operations seemed most promising. As a result, the Self-Care Operations were reduced to the productive type of operations. Thus, conceptually, measurement of Self-Care Agency with the A.S.A.-scale is though not strictly limited to yet mainly focused on the power to perform productive Self-Care Operations. 
Individual Self-Care Agency values can be described in terms of development, operability and adequacy. Adequacy presupposes developed and fully operative Self-Care Agency. Measurement of Adlequacy presumes knowledge of Self-Care Requisites, effective technologies and a program of discrete SelfCare Actions to be performed. Development and operability both can be measured in terms of the Self-Care Operations individuals consistently can perform. Measurement of development is based on the assumption that all human beings can be devided into classes of persons with undeveloped, developing and developed Self-Care Agency. Developed Self-Care Agency can and can not be operative, while operative Self-Care Agency presupposes developed Self-Care Agency. Thus, the purpose of the project being to create an instrument, that would be applicable to adults in various states of health, it was decided to measure operability of Self-Care Agency.

\section{Operationalization}

The next phase consisted of the operationalization of the variables. Empirical referents were generated for the various combinations of power components and productive Self-Care Operations. These are also called the form of SelfCare Agency (Orem, 1980, p.83). As the content of Self-Care Agency derives from meeting Self-Care Requisites, the Universal Self-Care Requisites were used as a frame of reference namely: intake of air, water, food, elimination care, activity-rest balance, solitude-social interaction balance, hazard prevention and normalcy promotion. The decision to generate empirical referents using the Universal Self-Care Requisites was influenced by the desire to create an instrument that would be generally applicable to adults in various states of health.

Initially, a totall number of 56 items were generated. These empirical referents were examined in terms of whether the referent reflected one or more of the power components. It soon became clear that the classification of power components did not consist of mutually exclusive categories. Nevertheless, a matrix was constructed with the empirical referents to determine whether a reasonable representation of all productive operations and all power components was reflected in the generated items. After a first screening of the items in terms of appropriateness to the Self-Care Agency concept by nineteen nurses who were students of Orem's Theory, the number of items was reduced to 36 .

\section{Measurement}

The generation of empirical referents for the scale also involved choices about the nature of the instrument. A Self-Appraisal scale was selected for this project. Even though a Self-Appraisal scale has the disadvantage of high subjectivity, the danger of systematic under and over estimation as well as social 
desirability tendencies, the individual's Self-Appraisal seemed to be the most obvious informative source as far as Self-Care Agency is concerned. In the Quality of Life research literature Campbell, Converse and Rogers (1976) have argued that subjective indicators assess the experience directly, whereas objective ones (like socio-economic indicators) merely measure things that influence the experience. Though Quality of Life is a different concept from Self-Care Agency, in terms of measurement a similar argumentation seems possible. Furthermore, biases can be mitigated by cross validation with significant others. With this thought in mind two forms of the scale were developed: a self-appraisal scale (Form A) and an appraisal of another scale (Form B), which can be completed by a spouse, significant other or nurse.

As scaling model the Likert approach has been choosen, because this procedure is 'Subject-Centered", that is its purpose is to scale respondents and not items. All systematic variation in the responses is attributed to differences among respondents (McIver, Carmines, 1981, p.23). Five response categories have been choosen ranging from Totally Disagree, Disagree, Neither Disagree Nor Agree, Agree, Totally Agree with numerical values climbing from ' 1 ' (Totally Disagree) to ' 5 ' (Totally Agree). The weighting scheme is reversed for unfavorable or negative statements, so that higher scores always indicate more operable Self-Care Agency. Individual items are combined into a summative scale. Nunnally (1978, p.67) suggests as the importance of this additive model, that it averages out random error involved in individual item measurement. It is further assumed that each item is monotonically related to operability of Self-Care Agency. The more operable a respondent's Self-Care Agency the higher his/her expected score for the items. This assumption does not imply that each item has the same relationship with operability of Self-Care Agency. It is further assumed that the items as a group measure only operability of Self-Care Agency. All items to be linearly combined should only be related to a single common factor. The sum of the items is expected to contain all important information contained in individual items (Mc Iver, Carmines, 1981, p.23). Scale scores are computed by simply summing the response scores of the component items. Nunnally $(1978, p .606)$ argues for such parsimonious treatment of component items for two reasons. He finds it difficult to defend other arbitrary systems of weighting in comparison to an equal weighting scheme. But more importantly he also finds little gain from the effort: unweighted and weighted summative scores regularly correlate quite highly. Measurement of operability of Self-Care Agency will be considered as done at an interval level.

Conceptually, measurement of Self-Care Agency with the A.S.A.-scale is though not strictly limited to yet mainly focused on the power to perform productive Self-Care Operations. Furthermore operability of Self-Care Agency is measured. Universal Self-Care Requisites were used as frame of reference in the operationalization phase. Empirical referents were gemerated for the 
various combinations of Power-components and Self-Care Operations. At the end of this phase the number of items was 36 . For this project a self-appraisal scale was selected (Form A.S.A.-A). An additional form has been deweloped: an appraisal of another scale (Form A.S.A.-B) which with the purpose of cross-validation can be completed by a spouse, significant other or nurse. A Likert type of scaling is used with five response categories ranging from Totally Agree (numerical value 1) to Totally Agree (numerical value 5). Individual items are combined into an unweighted summative scale.

\subsection{Content Validation}

Content validity depends on the extend to which an empirical measurement reflects specific domain of content (Carmines and Zeller, 1979, p.20). Support for content validity is derived from subjective judgement that the items are representative of the content area. Content validity testing of the A.S.A. has been done in the U.S.A. by a panel of eight nurse experts. They were either doctorally prepared faculty members or doctoral students working within Orem's conceptual framework in their research or practice. Experts were asked to rate each item for its positive or negative nature and for the extend to which the item reflected the concept Self-Care Agency. Criteria for inclusion of the items in the ultimate scale were: $100 \%$ agreement on its nature; more than $50 \%$ agreement on its good reflection of the concept, and type of power component in the item. To insure even distribution of all power components of Self-Care Agency in the scale, at least two items that represented each of the ten power components and met the first two criteria were included in the scale.

On the basis of expert opinions, the total number of items was reduced from 36 to 24 . Fifteen statements were positive and nine were negative. The 24 items were assessed as providing satisfactory representation in the scale of the ten power-components and 5 Self-Care Operations (see table 2.1.). For scoring purposes all negative item values will be reversed.

The ordering of the items has been established at random.

Examples of items included in the self-appraisal (Form A) in its present form are

'I rarely check whether the measures I take to stay healthy are adequate'

'I look for better ways to take care of myself'

Example items included in the appraisal of another (Form B) scale are:

'Rarely checks whether the measures taken to stay healthy are adequate'

'When taking new medication, obtains information about the side effects'.

D.E. Orem (1986) in personal communications contributed editorial comments for the refinement of some of the items. In a second series of personal communications (1988) D.E. Orem stated as her overall conclusion that the A.S.A.-scale measures 'Activation of Power' and 'Engagement in Self-Care 
Table 2.1. Distributhow of Power Components and Self-Care Operation un A.S.A.scale.

\begin{tabular}{|c|c|c|c|}
\hline ASA-item nir. & Homalture & Power Component & Self-Care Operation \\
\hline 1 & + & IV & 10 \\
\hline 2 & $\because$ & IV & 8 \\
\hline 3 & + & III & 7 \\
\hline 4 & + & $x$ & 7 \\
\hline 5 & + & IX & 10 \\
\hline 6 & - & II & 7 \\
\hline 7 & + & $\mathrm{v}$ & 9 \\
\hline 8 & + & $X$ & 9 \\
\hline 9 & + & I & 9 \\
\hline 10 & + & VIII & 7 \\
\hline 11 & - & VI & 7 \\
\hline 12 & + & VIII & 8 \\
\hline 13 & - & II & 7 \\
\hline 14 & * & VII & 6 \\
\hline 15 & . & $\mathbb{I}$ & 7 \\
\hline 16 & + & VII & 7 \\
\hline 17 & + & V & 10 \\
\hline 18 & + & VIII & 8 \\
\hline 19 & + & I & 10 \\
\hline 20 & - & VI & 6 \\
\hline 21 & + & VII & 10 \\
\hline 22 & + & VI & 7 \\
\hline 23 & - & VI & 7 \\
\hline 24 & - & III & 7 \\
\hline
\end{tabular}

Operations'. 'Given the answers to the items inferences can be made about which power components are operable and which are not. If they are operable they are developed. If they are not operable nothing can be infered in relation to development". She further commented. 'Items reflect clusters of powercomponents and many items reflect more than one power-component'. In conclusion the A.S.A.-scale seems to be reflective of operability of Self-Care Agency.

\subsection{Translation}

Both forms have been translated into the Dutch language by three bilingual faculty of the University of Limburg, The Netherlands, who also participated in the project of instrument devellopment. To reach consensus on the definitive version individual translations were discussed and compared in a group session. Translation of the scale necessitated cross-cultural comparison of the content of the statements. Item $\mathrm{nr} .8$ generated ample discussion about cultural differences in the frequency of bathing and showering; item nr. 9 about the differences 
in average body weight; item nr. 11 about possible differences in valuing kinds of exercise on a daily routine and items $\mathrm{nr} .14,15,16$ about cultural differences in client roles. While translating safety it was realized that there is only one word in Dutch to translate both 'safety' and 'security' namelly 'veiligheid'. Literal translation of 'due to limited mobility' ( $\mathrm{nr}$. 24) 'beperkte mobiliteit' was rejected for this was considered technical health professional's language. It was decided to translate into "door mijn lichaamsbouw".

For the purpose of counter check in 1987 three professional translators of the Language Institute related to University of Limburg were asked to retranslate the Dutch version of the scale into English. The translators were not given the original English version of the instrument. Afterwards the results were discussed with the translators. The Dutch word zelfzorgvermogen was translated as Self-Care Capabilites. This is obvious since Self-Care Agency is a technical term. Item nr. 24 was translated in 'Due to my physical condition' or in terms of "body build" and as such not accurately reflecting the original English 'Due to limited mobility'. It was an observation of the translators that the Dutch translation of item $\mathrm{nr}$. 10 caused ambiguity in interpreting its meaning. This was reflected in the three translations of 'When needed I manage to be by myself' a) 'If necessary I can be alone', b) 'When mecessary I can be on my own', c) 'When necessary I am capable of being alone'. One of the translators, being a bilingual of British origin draw attention to the important semantic difference in the meaning of 'sanitary conditions' item nr. 4 between American and Queen's English. As a last step in this process three native born unilingual English speaking U.S. residents were asked to compare the three translations with the original A.S.A.-scale. Each original item as well as each translated item was broken down into two or three units. The units of the original item were comparable with the units of the three translations. Raters were asked to judge each item in terms of the accuracy of the translation and the 'goodness' of the grammar. They could rate the translated unit as comparable to the original as better or as worse than the original. When agreement between at least two raters is used as a criterion for agreement than in $4 \%$ of the 201 translated item units there was no agreement. Fourty seven percent of the translated units were considered worse, $32 \%$ as comparable and $17 \%$ as better . Except for item nr. 24 differences of translations in relation to the original had to do with the formulation and not the content. In conclusion results of this retranslation of the translated Dutch version into English revealed that content whise the Duch version of the A.S.A.-scale is fully comparable with the English version with the exception of item $\mathrm{nr}, 24$. 


\section{HYPOTHESES}

\section{Introduction}

Reliability and validity of existing instruments to measure Self-Care Agency have been analysed in chapter one. In terms of relliability testing adequacy of sample choises and sample sizes was questioned. In terms of construct validity lack of hypotheses testing based on Orem's theory was criticized. As the purpose of this study it was stated to test reliability and validity of a new instrument developed to measure Self-Care Agency as defined by Orem. Reliability and validity testing of the A.S.A.-scale is the subject matter of the remainder of this study.

Definition of and methods to test reliability and validity are explored in this chapter. Specific hypotheses are formulated to test internal consistency, equivalence and stability of the A.S.A.-scale. Based on Orem's Self-Care Deficit Theory for nursing hypotheses are formulated to test for construct validity. This chapter ends with some hypotheses regarding the influence of measurement conditions on reliability and validity.

These hypotheses were generated within the context of a program of seven research studies. The first one of these studies deals with a population of elderly, the second with non-institutionalized arthritis patients and study three with patients institutionalized in a rehabilitation center. Study four and five refer to patients respectively staying in and discharged from acute care hospitals. Study six exclusively focuses on patients undergoing coronary artery bypass surgery. The last study deals with a sample of young healthy college students. Hypotheses formulated in this chapter need to be seen in relation to these studies. It is mainly for reasons of systematic description that this chapter contains all generated hypotheses, while chapter four describes the method of the studies.

\subsection{Reliability and Validity of Measurements}

Measurement has been defined as the 'process of linking abstract concepts to empirical indicants' (Carmines and Zeller, 1979, p.10). This definition makes 
clear that measurement is a process involving both theoretical as well as empirical considerations. From an empirical standpoint the focus is on the observable response: the indicator. Theoretically interest lies in the underlying unobservable and directly unmeasurable concept in relation to other concepts.

Given this definition of measurement reliability concerns the degree to which results are consistent across repeated measurements (Carmines and Zeller, 1979). A measuring instrument is relatively reliable if it is minimally affected by random measurement error (Kerlinger, 1973). Synonyms for reliability are dependability, stability, consistency, accuracy (Kerlinger, 1973) and equivallence (Polit and Hungler, 1987). Validity is evidenced by the degree that a particular indicator measures what it is supposed to measure, rather than reflecting some other phenomenon i.e. non random measurement error (Kerlinger, 1973, Carmines and Zeller, 1979). Strictly speaking one validates an interpretation of data arising from a specified procedure (Cronbach, 1971). Apart from content validity, which has been addressed in the second chapter, criterion related validity and construct validity are identified as basic types of validity. Both Kerlinger (1973) and Carmines and Zeller (1979) state that criterion related validity has limited usefullness for assessing validity of empirical measures of theoretical concepts employed in the social sciences. Usually no 'objective' standard is available. Thus construct validity is used with preference in the remainder of this study. Essentially construct validation focuses on the extent to which a measure performs in accordance with theoretical expectations (Carmines and Zeller, 1979). It unites psychometric notions with theoretical notions by empirically testing hypotheses derived from the theory involving the construct (Kerlinger, 1973). Support for construct validity can be accumulated by testing theory derived hypotheses in different studies in many ways.

Strictly speaking the reliability and validity of an instrument are not a property of an instrument, but rather of an instrument when administered to a certain sample under certain conditions for a certain purpose (Polit and Hungler, 1987). The reliability and validity of a measuring instrument are not totally independent. Reliability is a necessary but not sufficient condition of the value of research results and their interpretation. High reliability is no guarantee of good scientific results but there can be no good scientific results without a certain degree of reliability (Kerlinger, 1973). The relationship between reliabillity and validity has also been expressed in terms of variance (Guilford, 1954, Kerlinger, 1973). When a measurement instrument is administered to a sample total variance of the scores can be calculated. This total obtained variance includes systematic or "true" and error variance. As it is possible to estimate error variance reliability can be expressed as the proportion of total variance minus error variance to total variance. In a similar way validity can be defined as the proportion of common factor variance to total variance of a measure. Common factor variance or 'communality' is the 'true' variance of a measure that is shared with other measures. In contrast to the common factor variance of 
a measure is its specific variance: the variance that is not shared by any other measure. In summary a measurement's total variance is composed of common factor variance, specific variance and error variance. Talking in proportions of total variance this is expressed in the equation:

$\frac{V t}{V t}=\frac{V c o}{V t}+\frac{V s p}{V t}+\frac{V e}{V t}$

where $\mathrm{Vt}=$ total obtained variance, $\mathrm{Vco}=$ commonfactor variance, $\mathrm{Vsp}=$ specific variance and $\mathrm{Ve}=$ error variance.

The part of the equation expressing validity is $\frac{V c o}{V t}$, while the part of the equation expressing reliability is $\frac{V c o}{V t}+\frac{V s p}{V t}$ As such reliability and validity are seen as parts of one unified whole.

Definition of reliability can also be approached in the following way. If we measure the same set of objects again and again with the same comparable measuring instruments, we need to get the same or similar results (Kerlinger, 1973). A first method used to estimate an instruments reliability is the internal consistency method. An instrument may be said to be internally consistent or homogeneous to the extent that all of its sub-parts are measuring the same characteristic (Polit and Hungler, 1987). A second approach to estimate the reliability of a measure is called equivalence testing (Polit and Hunger, 1987). This is the case when different observers or researchers are using an instrument to measure the same phenomena at the same time or when two presumably parallel instruments are administered to individuals at about the same time. In both situations the aim is to determine the equivalence of the instrument(s) in yielding measurements of the same traits in the same subjects. The stability of a measure as a third approach to estimate reliability refers to the extend to which the same results are obtained on repeated administrations of the instrument (Polit and Hungler, 1987). The estimation of reliability here focuses on the instrument's susceptability to extraneous factors from one application to the next.

Construct validity refers to the extent to which a measure performs in accordance with theoretical expectations (Carmines and Zeller, 1979). The instrument designer adopting this approach is concerned with the questions: What is this measuring device really measuring? Is the abstract concept under investigation being adequately measured with this instrument? (Polit and Hungler, 1987). One common approach to construct validation is the Known Groups Technique (Kerlinger, 1973). Groups of people with 'known' characteristics are administered the instrument and differences and the direction of differences of scores are predicted and tested. A second approach to construct validation is Demonstration of Convergence and Discriminability. Evidence 
that different methods of measuring a construct yield similar results is called Convergence (Kerlinger, 1973). Discriminability refers to evidence that one empirically can differentiate the construct under study from other constructs that may be similar but not or negatively related (Kerlinger, 1973). A third approach to construct validation consists of examination of relationships based on theoretical predictions (Pollit and Hungler, 1987). Kerlinger (1973) refers to it as deriving hypotheses from the theory involving the construct and testing the hypotheses empirically. As a last approach to construct validation factoranalysis can be mentioned (Carmines and Zeller, 1979). Factor-analysis refers to a variety of statistical techniques whose common objective is to represent a set of variables in terms of a smaller number of hypothetical variables (Kim and Mueller, 1978). At one extreme a researcher may use factor-analysis as a means of exploring data for possible data-reduction without having specific idea's about the number of underlying dimensions of a measurement instrument. This form of use is called exploratory factor-analysis. On the other extreme a researcher may anticipate certain underlying dimensions in a measurement instrument and expect that certain variables belong to one dimension, while others belong to other dimensions. If factor-analysis is used to test this expectation it is refered to as confirmatory factor-analysis (Kim and Mueller, 1978).

\subsection{Internal Consistency}

Consistency or accuracy deal with the question whether the measures obtained are 'true' measures of the property. As such reliability is defined as the relative absence of errors of measurements or as the proportion of error variance to the total obtained variance subtracted from 1.00 (indicating perfect reliability). This can be calculated as a reliability coefficient (Kerlinger, 1973) and is refered to as the internal consistency method. Cronbach's alpha is the most popular and usefull index (Carmines and Zeller, 1979, Polit and Hungler, 1987). Thus the internal consistency of the A.S.A.-A, when administered to different study populations, will be examined by calculating Cronbach's alpha. There is no absolute standard for what a reliability coefficient should be. It is suggested that for making group level comparisons coefficients in the vicinity of .70 or even .60 would probably be sufficient. If, however, measures are to be used as a basis for making decisions about individuals then the reliability coefficient should be $\geq .90$ (Polit and Hungler, 1987). For the purpose of internal consistency testing the first hypothesis is formulated as follows.

H.1. Cronbach's alpha's of measurements with the A.S.A.-A scale will be $\geq .70$.

The A.S.A.-scale is a summative scale of a Likert Type. The items as a group measure operability of Self-Care Agency. It is assumed that all items linearly combined are only related to a single common factor. Thus consistent and accurate measurement with the A.S.A.-scale presupposes homogeneity or 
unidimensionality of the instrument. Two types of item analysis have been suggested to evaluate the ability of the individual items to measure the attribute by the total scale. The first is the computation of item to total correlations (Mc Iver and Carmines, 1982). It can be assumed, that items which have a corrected item to total correlation of $<.30$ do not contribute to undimensionality or homogeneity of the instrument. The second is the critical ratio or t-test which evaluates subgroup mean differences relative to item score variances (mc Iver and Carmines, 1982). It provides an indication of the degree to which an item differentiates between high and low subgroups. This can be tested by comparing 25\% of the lowest Total A.S.A.-A scores with 25\% of the highest Total A.S.A.-scores. Items are analysed by comparing the difference in mean item score of the group lowest total A.S.A.-A scores and the group highest total A.S.A.-A scores using a t-test. It can be assumed that items with a nonsignificant value on this $t$-test do not contribute to homogeneity of the instrument. Based on these criteria the following hypotheses are formulated.

H.2. Item to total correlations of all 24 items of the A.S.A.-A scale will be $\geq .30$.

H.3. X item score of all 24 items of the group of $25 \%$ highest Total A.S.A.-A scores will be significantly higher than $\bar{x}$ item score of these items of the group of $25 \%$ of the lowest Total A.S.A.-A scores.

Items that have corrected item to total correlation of $<.30$ and items that don't discriminate significantlly on a one tailed t-test between high and low Total A.S.A.-A scores can be dropped to increase reliability. Dropping items could affect validity of the instrument. Items retained in the scale will be compared with items that are discarded in terms of power components reflective of operability of Self-Care Agency.

It is suggested not to rely on either one method in reliability testing and to use also factor analysis as a next step (Mc Iver and Carmines, 1982). Several aspects of the extracted unrotated factor matrix can evaluate single phenomenon measurement. 1) The first extracted component should explain a large proportion of the variance in the items. 2) Subsequent components should explain fairly equal proportions of the remaining variance except for gradual decrease. 3) All or most of the items should have substantial loadings on the first component (Carmines and Zeller, 1979). It can be assumed that the first component of the unrotated matrix of the factor analysis of the A.S.A.-A scale will count for twice the percentage of variance of the second component, while the percentages of the second and next one's will be comparable with some gradual decrease. An item to factor correlation (loading) of $\geq .35$ on the first component will be used as a critical indicator. Based on these assumptions and criteria the following hypotheses are formulated.

H.4. The percentage of explained variance of the first component will be twice the percentage of explained variance of the second component on the unrotated matrix of the factor analysis of the A.S.A.-A scale. 
H.5. Item to factor correlations (loadings) of all 24 items on the first extracted component of the unrotated matrix of the factor analysis of the A.S.A.-A scale will be $\geq .35$.

\subsection{Equivalence}

Consistency or accuracy can also be evaluated by comparing different measurements of the same attribute in the same subjects. This kind of reliability is refered to as equivalence. When different observers are using an instrument to measure the same phenomenon at the same time, this is called inter-observer reliability. When presumably parallel instruments are administered to individuals at about the same time, one speaks of alternative form or parallel instrument measurement. Parallel measurements are functions of the same time scores and the differences between them are the result of purely random error (Carmines and Zeller, 1979). The estimation of reliability can be calculated using analysis of variance or a correlation coefficient between parallel measures scores.

As described in chapter two the A.S.A.-scale was originally developed as a self-appraisal scale: A.S.A. form A. Strictly speaking it is not possible to establish inter-observer reliability of a self-appraisal scale, because different observers cannot use an identical instrument to measure the same phenomenon at the same time. In order to evaluate equivalence a second form of the instrument was developed: an appraisal of another scale: A.S.A.-B. This scale can be completed by a significant other for instance spouse, or by a nurse. To evaluate equivalence of the scale study subjects" scores given by themselves on the A-form can be compared with their scores on the B-form of the scale given by significant others and/or nurses the latter in case of patients being the study subjects.

The first proposition of the theory of Self-Care states that 'Self-Care is learned within the context of social groups by human interaction and communication (Orem, 1980, p.28). Based on this proposition it is expected that significant others by nature of definition being important members of a subject's social group can make valid inferences of subject's operability of Self-Care Agency by interaction and communication. A positive correlation may be expected between study subject's Total A.S.A.-A scores and these subject's Total A.S.A.-B scores given by their significant others. Expected positive correlations between nurses' A.S.A.-B and their patient's A.S.A.-A scores are based on proposition nr. 5 of the theory of Nursing Systems: 'Nurses determine the current and changing values of patients abilities to meet their self care requisites using specific processes or technologies' (Orem, 1980, p.30). One of the technological orientations and operations in nursing practice is described as diagnosis of the values of the constituent elements of Self-Care Agency of the patient in terms of operability (Orem, 1980, p.91). Social and interpersonal technologies like communication adjusted to age, developmental state, health 
state and to social cultural orientation and bringing about interpersonal relations are refered to as specific technologies nurses use to determine Self-Care Agency (Orem, 1980, p.90). Thus it may be expected that nurses can make valid inferences of a patient's operabilty of Self-Care Agency.

It is expected that both relations can be expressed as significant moderate positiwe correlations Pearson's correlation coefficients ranging from $r=.30$ to $r$ $=.70$. Higher correlations are not expected because self-appraisal scores cannot be considered identical to appraisal of another scores. Lower scores seriously question equivalence of the A.S.A.-scale. Based on this argumentation the following hypotheses can be stated.

H.6. Subjects" Total A.S.A.-A scores will be significantly positively $(r>30$, $<.70)$ correlated to these subjects' Total A.S.A.-B scores given by their significant others.

H.7. Patients' Total A.S.A.-A scores will be significantly positively $(r>.30$, $<.70$ ) correlated to these patients' Total A.S.A.-B scores given by nurses, who take care of these patients on a regular basis.

One tailed testing of significance will be practiced. Expected positive correlations between A.S.A.-A and A.S.A.-B scores are based on propositions and presuppositions of Orem's theories. As such evaluation of equivalence by means of parallel instrument measurement can also be considered as a form of construct validity testing.

\subsection{Stabillity}

Stability, dependability or predictability deal with the question whether measurement of the same phenomenon again and again with the same instrument leads to the same or similar results. Assessments of the stability of a measuring tool are derived through procedures refered to as test-retest reliability (Kerlinger, 1973; Carmines and Zeller, 1979; Polit and Hungler, 1987). Stability of repeated measurements can be evaluated in terms of covariation of individual A.S.A.-A scores. It seems usefull to evaluate high, medium and low total A.S.A. A scores if possible as separate subgroups to detect instability between these subgroups. Orem on the one hand emphasizes stability of Self-Care Agency. Loss of Self-Care Agency may result from health-derived or health-related limitations. On the other hand development and inherently change is another major concept in the model. Changes in Self-Care Agency are necessary for survival as the person matures. According to Fawcett (1984) these two elements do not present a logical inconsistency. Persistence and change of Self-Care Agency are combined by Orem by emphasizing, that Self-Care Agency is conditioned by age, developmental and health state. Thus in testing for stability of Self-Care Agency subjects experiencing major critical life-events within test time-intervals should be excluded from tests. It is expected that A.S.A.-A scores of individuals correlate strongly in a positive direction Pearson's correlation coefficient being in the vicinity of $r=.70$ for short time 
interval testing. These results should be consistent for low, medium and high A.S.A.-A scores. For longtime interval testing correlation between indiwidual A.S.A.-A scores may be only moderate due to time induced change. Again stability of results should be evaluated for low, medium and high A.S.A.-A scores. To test for stability of the A.S.A.-A scale the following hypotheses are formulated. One tailed testing of significance will be practiced.

H.8. Subjects' Total A.S.A.-A scores will be significantly strongly and positively $(r \geq .70)$ correlated when measured with a six weeks time-interval in which no major critical life events were experienced by them.

H.9. Subjects" Total A.S.A.-A scores will be significantly moderately and positively $(r \geq .40)$ correlated when measured with a six months time interval in which no major critical life events were experienced by them.

\subsection{Construct Validity}

Construct Validity is central to the measurement of abstract theoretical concepts (Carmines and Zeller, 1979). Construct validation can be approached in several ways, but there is always the preoccupation with theory and the testing of hypothesized relations (Kerlinger, 1973; Polit and Hunger, 1987). Self-Care Agency can be seen as one of the core concepts of Orem's Self-Care Deficit Theory. The following propositions constitute this theory.

Prop. nr.1.Persons who take action to provide in their own Self-Care have Self-Care Agency.

Prop. nr.2.Self-Care Agency is conditioned by age, developmental state, life experience, socio-cultural orientation, health state and available resources.

Prop. nr.3.The relationship of persons' Self-Care Agency to the qualitative and quantitative Self-Care Demand can be determined when the value of each is known.

Prop. nr.4.The relationship between Self-Care Agency and Self-Care Demand can be defined in terms of equal to, less than, more than.

Prop. nr.5.Nursing is a legitimate service when

a) there is a deficit relationship between Self-Care Agency and Self-Care Demand

b) there is no deficit relationship between Self-Care Agency and Self-Care Demand, but a future deficit relationship can be expected because of predictable decrease of Self-Care Agency, qualitative or quantitative increase of Self-Care Demand, or both.

Prop. nr.6.Persons with existing or projected Self-Care Deficit are in or are expected to be in states of social dependency that legitimate a nursing relationship (Orem, 1985, p.35).

Based on these theoretical notions construct validation of Self-Care Agency is 
approached by means of the 'Known Groups Technique', by means of 'Demonstration of Convergence and Discriminability' and by 'Examination of proposed Theoretical Relationships".

\section{Known Groups Technique}

One common approach to construct validation is the Known Groups Technique. In this procedure groups of people with 'known' characteristics are administered the A.S.A.-A scale and differences and the direction of differences of scores are predicted. Orem's Self-Care Deficit Theory states that persons who take action to provide their own Self-Care have Self-Care Agency (prop: nr. 1). The relationship between Self-Care Agency and Care Demand can be defined in terms of equal to, more than or less than the latter being Self-Care Deficit (prop. nr. 4). Persons with existing Self-Care Deficits are in states of sociall dependency that legimate a nursing relationship (prop. nr. 6). The Self-Care Deficit Theory rests on a number of pre-suppositions. Relevant in this respect is the following. Societies provide for the human states of social dependency by instituting ways and means to aid persons according to the nature of and the reason for their dependency. For the rapidly growing population of elderly Dutch society has institutionalized degrees of social dependency on nursing and other forms of care, housekeeping included, in different institutions. Since a few decades a 'Nursing Home' (Verpleeghuis) provides integrated professional nursing, medical and psychosocial care as well as complete hotel service to chronic and terminal patients. A 'Personalized Care Facility' (Bejaarden-Verzorgingstehuis) provides (professional) nursing care plus complete hotel service to elderly limited in respect to Activities of Daily Living. A 'Service Flat' provides accomodation, limited housekeeping and meal-service as well as the possibility of direct nursing assistance in case of an emergency to elderly who are at risk or somewhat limited in terms of Activities of Daily Living. Together with elderly living independently in the community residents of these institutions constitute four groups who can be expected to differ as far as dependency on care is concerned. The direction of the differences in dependency between the four groups goes from low for the elderly living independently in the community to high for the residents of the nursing home.

To test construct validity of the A.S.A.-A scale the following hypotheses will be tested.

H.10. $\tilde{\mathrm{x}}$ Total A.S.A.-A score of Elderly living independently in the community will be significantly higher than $\bar{x}$ Total A.S.A.-A score of ServiceFlat-residents.

H.11. $\tilde{\mathrm{x}}$ Total A.S.A.-A score of Service-Flat-residents will be significantly higher than $\tilde{\mathbf{x}}$ Total A.S.A.-A score of Personalized Care FacilityResidents.

H. 12. $\overline{\mathrm{x}}$ Total A.S.A.-A score of Personilized Care Facility Residents will be 
significantly higher than $\bar{x}$ Total A.S.A.-A score of Nursing Home Residents.

T-test for independent samples will be used to test the separate hypotheses. One tailed testing of significance will be practiced. Analysis of covariance will be used to compare the difference between $\bar{x}$ Total A.S.A.-A scores of the four groups in relation to relevant other variables.

\section{Demonstration of Convergence and Discriminability}

A second approach to construct validation is refered to as Demonstration of Convergence and Discriminability. Convergence refers to evidence that different methods of measuring a construct yield similar results (Kerlinger, 1973; Polit and Hungler, 1987). As described in chapter one Kearney and Fleischer (1979) developed the Exercise of Self-Care Agency Scale ESCAS as a 43 item self-appraisal instrument to measure Self-Care Agency. Content-analysis of this scale (Isenberg, 1986) resulted in the conclusion that six items are reflective of the Power elements of Self-Care Agency, and 10 items are expressions of Self-Care Operations. As such this 16 item ESCAS and the A.S.A.-A can be considered different measurements of elements of the same construct. Thus administered to the same population a positive correlation can be expected. As the ESCA scale was developed using the inductive approach to identify components of exercise of Self-Care Agency measurement with this scale may not cover the entire content of the concept. The A.S.A.-scale was developed based on analysis of the concept Self-Care Agency as described by Orem and N.D.C.G.. A systematic attempt was made to cover all power-components and all productive Self-Care Operations. Thus correlation between shortened version of ESCA and A.S.A. can be expected to be moderate but not necessarily strong. The following hypothesis will be tested practicing one tailed testing of significance.

H.13. Subjects' Total A.S.A.-A scores will be significantly positively and moderately ( $r \geq .40)$ correlated to Total Shortened Version (16 items) ESCAS scores.

A very simple method to measure Self-Care Agency is the question 'How do you assess your capability to care for yourself?" with the structured answering possibilities very bad, bad, moderate, well and very well. The answers can be given a numerical value from 1 to 5 . This one-sentence General Assessment of Self-Care Agency from a psychometrical point of view is inferior to a multi-item A.S.A.-A scale. Mc Iver and Carmines (1982) give the following reasons for this. It is very unlikely that a single item can fully represent a complex theoretical concept. Secondly single-item measures lack precision. Thirdly single-item measures are more prone to random error than multi-item measures, because random error averages out when scores on numerous items are summed to obtain a total score. Nevertheless a positive correlation should be expected between such single and multi-item measurement like the General Assesment 
of Self-Care Agency and Totall A.S.A.-A scores. Thus the following hypothesis will be tested practicing one tailed testing of significance.

H.14. Subjects Total A.S.A.-A scores will be sígnificantly positively and weakly to moderately $(r \leq .40)$ correlated to General Assessment of Self-Care Agency-scores.

Discriminability refers to evidence, that one empirically can differentiate the construct under study from other constructs that may be similar, but not or negatively related (Kerlinger, 1973). Being exposed to Orem's idea's at first many people's reaction is that Self-Care Agency seems similar to the construct of 'Activities of Dailly Living' 'A.D.L.'. Katz' $(1963,1976,1979)$ well known Index of Independence of Activities of Daily Living was developed as a measure of function to evaluate results of treatment of chronically ill and aged patients. A.D.L. refers to activities which people perform habitually and universally. Originally the index was developed from observations of a large number of activities performed by a group of patients with fractures of the hip (Katz e.a. , 1963). With this instrument degree of independent functioning in terms of bathing, dressing, transfer, eating, toiletting and continence can be investigated with chronic patients and elderly. Independent is defined as functioning without supervision, advise or assistence and actual functioning is the focus of investigation. Both degree of independent functioning can be calculated for the six separate functions as well as total so called A.D.L.-functioning. The higher the score the greater the amount of dependence in the functions. As such this instrument is called a measure of primary sociobiological functions. Conceptually A.D.L. in many ways is a different idea than Self-Care Agency. A.D.L. refers to performance, Self-Care Agency to capability to perform; A.D.L. refers to six mainly bodily functions, Self-Care Agency to estimative, transitional and productive operations directed to fullifilment of eight both physical and psychosocial universal care requisites plus a number of developmental and health-related requisites. As such A.D.L. is a much more concrete concept than Self-Care Agency. A.D.L. refers to degree of independent functioning while Self-Care Agency can be asssessed in terms of degree of development, operability and adequacy. In conclusion although at first glance there may seem to be some similarity between A.D.L. and Self-Care Agency a more thorough comparison leads to the conclusion that both concepts stand for different, hardly related idea"s. Thus it may be expected that empirically they also are not or at the outmost weakly related. The following hypothesis will be tested practicing two tailed testing of significance.

H.15. Chronic patients and elderly patients' Total A.S.A.-A scores will not be significantly correlated with these patients' Total A.D.L.-scores.

\section{Examination of Relationships}

Another approach to construct validation consists of an examination of 
relationships based on theoretical predictions (Polit and Hungler, 1987). The first proposition of Orem's Self-Care Deficit Theory states that persons who take action to provide their own Self-Care have Self-Care Agency (Orem, 1980, 1985). Thus a positive relation can be hypothesized between Self-Care Agency and Self-Care. The theory of Self-Care further states that Self-Care is deliberate action sequentially performed to meet known care requisites (prop. nr. 2). Some of these requisites are common to all human beings: Universal Self-Care Requisites (prop. nr. 4). Eisler, Wolf and Diers (1972) have developed a self-report measure to assess patient's evaluation of his condition from day to day as he recuperates from surgery. The items of this so called Patient Recovery Inventory (P.R.I.) are concerned with sleeping, eating, urinating, mobility and so forth. The patient is asked to rate each item from very poor to excellent. Ratings are summed for a total score. The instrument was revised to measure the degree in which all Universal Self-Care Requisites are met by patients. This so called Revised Patient Recovery Inventory (R.P.R.I.) is a Likert-type self-appraisal scale with which patient's Self-Care in relation to Universal Self-Care Requisites can be measured. Thus the following hypothesis will be tested practicing one tailed testing of significance.

H.16. Patients' Total A.S.A.-A scores will be significantly positively correlated to their Total R.P.R.I -scores.

The second proposition of Orem's Self-Care Deficit Theory states that the individual's Self-Care Agency is conditioned by age, developmental state, life-experience, socio-cultural orientation, health and available resources (Orem, 1980, 1985). As already mentioned in chapter two these basic conditioning factors, in contrast to the human capabilities and dispositions, don't explain the structure of Self-Care Agency but condition its value (N.D.C.G., 1979). Operability of Self-Care Agency is affected by genetic and constitutional factors, culture, life experience and health state (Orem, 1980). Health state is used in the sense of a state of a person that is characterized by soundness or wholeness of developed human structures and of bodily and mental functioning (Orem, 1985, p.179). Two points of view about human beings afford considerable meaning to health state namely that of human beings as persons and that of the structural and functional differentiation of human beings. The latter has been developed by various human and life sciences resulting in identification of modalities of functioning like bodily functions, mental functions and psychosocial functions. An individual's health state when used in the compound sense can be investigated along one or more of these dimensions. If health state conditions the walue of Self-Care Agency, positive change in health state should be followed by increase in operability of Self-Care Agency. A medical procedure, of which the most important effect is stated to be improvement of functional health or health state, is Coronary Artery Bypass Surgery (C.A.B.S.). In treatment of Cardiovascular Disease, which still is the prime cause of death in Dutch Society (C.B.S., 1986), open heart surgery takes an 
important place. The number of C.A.B.S. procedures still increases every year in the Netherlands. Effective revascularisation of the myocardium via coronary bypasses will improve cardiac output as an index of cardiovascular functioning. Cardiovascular functioning on its term can affect respiration, elimination and neurological functioning. As mentioned before Self-Care Agency is considered to be a relative stable attribute, so effects of change will take some time. Thus the following hypotheses can be generated.

H.17. C.A.B.S.-patients' 6 months post-operative $\overline{\mathrm{x}}$ Total A.S.A.-A score will be significantly higher than the same patients' pre-operative $\bar{x}$ Total A.S.A.-A score.

H.18. C.A.B.S.-patients' 12 months post-operative $\bar{x}$ Total A.S.A.-A score will be significantly higher than the same patients' pre-operative $\bar{x}$ A.S.A.-A score.

T-tests for dependent samples will be used to compare the difference of the $\bar{x}$ scores. One tailed testing of significance will be practiced.

Orem further proposes that Self Care is learned within the context of social groups by human interaction and communication. Kahn (1979) has described the kind of human interaction and communication through which affection is demonstrated and/or practices, perceptions and opinions are reinforced and/or symbolic or material assistance is provided to another person as Social Support. Individuals belonging to groups in which social support is highly valued and practiced may be positively influenced in terms of learning capabilities, orientation, self-understanding, self-awareness, self-image, self-value, self-acceptance and self-concerns. These human capabilities and dispositions on their term affect operability of Self-Care Agency (N.D.C.G., 1979). Again it should be remembered that Self-Care Agency is a relative stable attribute so change will take place over some time. Based on Kahn's definition an instrument has been developed to measure amount of Social Support: the Norbeck Social Support Questionnaire N.S.S.Q. (Norbeck; 1981). Amount of Social Support can be expressed in a Total Functional and Total Network score. Thus the following hypothesis will be tested practicing one tailed testing of significance.

H.19. Chronic patients' Total A.S.A.-A scores will be significantly positively correlated to Total Functional and Network N.S.S.Q.-scores.

In many cultures traditionally care giving has been linked primarily to women at home feeding and nurturing off spring (Leininger, 1984). In terms of sex stereotypes caring is seen in Dutch culture as a typical feminine quality as is emotionality and passiveness (Van der Veen, 1979; de Graaf, 1984). The masculine stereotype is characterized as independent, active, self-confident and well-balanced. Sex-stereotypes as part of a culture reflect general accepted opinions about typical qualities of males and females. Individuals' self-image is influenced by sex-stereotypes. One not only is born as but also educated to man and woman. Self-image is one of the dispositions thought to influence Self-Care 
Agency (N.D.C.G., 1979). Self-Care has been described as deliberate purposefull action to maintain life, human functioning and to promote development and well-being. Thus both masculine (well-balanced, active) and feminine (caring) qualities are required to Self-Care Agency. To measure sex-stereotyped self-image in Dutch culture an adjective check-list forming a masculine $\mathrm{M}$ and feminine $\mathrm{F}$ scale has been developed based on the Bem Sex Role Inventory (Bem, 1974; Bem e.a., 1976): Groningen Androgynie Schaal G.R.A.S. (de Graaf, 1984). Scores are combined to one score: the stereotype G.R.A.S. value. This is the t-value calculated as a result of statistically testing respondents difference in mean score on the M- and F-scale. Subjects with significant higher scores on the M-scale are classified as having a masculine stereotype self-image. The higher the negative stereotype G.R.A.S. value the more masculine the self-image is. Subjects with significant higher scores on the feminine adjectives are classified as having a feminine stereotype self-image. The higher the positive stereotype G.R.A.S-value the more feminine someone's self-image is. Subjects with scores above the median of both the M- and F-scale are classified as having an androgyne stereotype self-image. Subjects with low scores on both scales are classified as unclear. Based on this possible range of stereotype G.R.A.S.-values the following hypothesis will be tested in relation to sex-stereotyped self-image. One tailed testing of significance will be practiced.

H.20. Subjects' Total A.S.A.-A scores will be significantly but weakly positively or negatively $(r<30)$ correlated to Total G.R.A.S. scores.

Correiation coefficients between individual G.R.A.S.-item scores and Total A.S.A.-A-scores wil be calculated in order to further explore the way sexstereotyped self-image is related to Self-Care Agency.

Operability of Self-Care Agency presupposes development. Degree of development is based on the assumption that all human beings can be divided into classes of persons in terms of their Self-Care Agency. In adulthood Self-Care Agency attains a degree of perfection, but it declines with advancing age (N.D.C.G., 1979). Thus the following hypothesis will be tested practicing one tailed testing of significance.

H.21. Total A.S.A.-A scores of subjects older than 65 years will be significantly negatively correlated with their age.

\section{Factor-Analysis}

A last approach to contruct validation will be use of factor-analysis. As such this approach means a second and different use of factor analysis than its use for internal consistency testing. This procedure is used to identify and group together clusters of related variables. Each cluster called a factor represents a relatively unitary attribute within the instrument (Polit and Hunger, 1987). Self-Care Agency was conceptualised as a complex human characteristic. Ten 
distinct power-components of Self-Care Agency have been identified and used for the construction of the A.S.A.-A scale. These ten capabilities constituting the Power Component are considered by N.D.C.G. (1979) as formulations in need of review and testing. As mentioned before while operationalizing SelfCare Agency it became clear that the classification of the power-components did not consist of mutually exclusive categories. Content analysis further rewealed that itemis of the A.S.A.-A scale reflect clusters of power components and that many items reflect more than one power component. It can be expected, that clusters of power components will be reflected in the factor structure. Thus it will be evaluated to what extent clusters of power-components of Self-Care Agency are reflected in the extracted components of the rotated matrix of factor analysis done on total A.S.A. -A scores. Factor-analysis will be used both in an explorative and confirmatory way.

\subsection{Measurement Conditions}

Many conditions can contribute to errors of measurement. Among the most common are situational concomitants, response set biases, transitory personal factors, administration variations, instrument clarity, response sampling and instrument format (Polit and Hungler, 1987). Two last hypotheses will deal with measurement conditions.

A number of relatively enduring characteristics of respondents can interfere with accurate measures of Self-Care Agency. As mentioned in chapter two response set bias like social desirability is a potential problem in self report measures particularly scales. Response set bias concerns the tendency of certain individuals to respond to items in a characteristic way independently of the item's content. The social desirability response set is a bias stemming from a person's desire to appear in a favorable light. Crowne and Marlowe (1964) have developed a scale to locate individuals who describe themselves in faworable, social desirablle terms in order to achieve the approval of others. High scores indicate high social desirability. A Dutch adapted version of the scale has been developed and tested by Hermans (1976) and Visser (1984). A significant positive correlation would be indicative for response set bias. Robinson and Shaver (1975) have stated that response sets are seen as seldom obliterating item content but as seriously confounding the interpretation of scale scores in some content areas. Thus the following hypothesis will be tested practicing two tailed testing of significance.

H.22. Subjects' Total A.S.A.-A scores will not be significantly correlated to these subjects" Total scores on the adapted C. \& M. Social Desirability scale.

Scores can also be affected by conditions under which they are produced. In combination with social desirability response set the presence of the researcher 
could be a source of bias. This is refered to as the reactivity problem (Polit and Hungler, 1987). On the other hand it can be expected that the researcher's presence at data collection time is a better guarantee for standardized self reports without others contaminating self-appraisal scores. To demonstrate absence of measurement error in this respect the A.S.A.-A scale will be administered to a comparable sample in two different ways. The one way will be personally administrating the scale, the second sending the instrument by mail. No differences in mean scores are expected due to variation in this condition of measurement. Mean A.S.A.-A scores will be compared using t-test for paired samples. Two tailed testing of significance will be practiced. A significant t-test will be indicative for measurement error in this respect. Thus the last hypothesis can be formulated as follows.

H.23. $\overline{\mathrm{x}}$ Total A.S.A.-A score of subjects to whom the scale is personally administered will not significantly differ from $\tilde{x}$ Total A.S.A.-A score of comparable subjects to whom the scale is send by mail. 


\section{METHOD}

\section{Introduction}

An integrated research program has been developed to structure extensive reliability and validity testing of the A.S.A.-scale. (Evers, G.C.M., Isenberg, M. Philipsen, H., Brouns, G., Halfens, R., Smeets, H., 1986; Evers, G.C.M., Isenberg, M., 1987). Specific designs have been set up to be able to test the hypotheses formulated in chapter three. Care has been taken to select adequate samples. Variation has been sought in terms of demografics, health status, institutionalization status and socio-cultural differences. Studies include healthy subjects as well as subjects with health problems like coronary artery disease, arthritis, neurological problems e.a. Research populations include individuals living independently in the community, residents of a nursing home, a personalized care facility, a service flat, a rehabilitation center and acute care hospitals. Young adult and older males and females from urban, suburban and rural area's are represented.

First this chapter describes the integrated research program in terms of purpose, subjects and data analysis procedure of all studies combined. As such the chapter starts with a description of the procedure of meta-analysis of all studies involved. A total of seven studies are included in the program of research. The first study deals with a population of elderly, study II with non-institutionalized arthritis patients and study III with patients institutionalized in a rehabilitation center. Study IV and V refer to patients respectively staying in and discharged from acute care hospitals. Study VI exclusively focuses on patients undergoing coronary artery bypass surgery. The last study deals with a sample of young healthy college students. Purpose, design, sample selection and characteristics as well as instrumentation and procedure of data collection of these seven studies are described separately in the latter part in this chapter.

The studies I, II, III, IV and VII were specifically designed for the purpose of reliability and validity testing of the A.S.A.-A scale. (Schrijnemaekers, 1986; Boshouwers, 1987; Schiltmans, 1987; Kalter, 1987; Courtens, 1987 and van Achterberg, 1988). The authors collected data for the research program as part of the work of their masters theses. Study $V$ was carried out as part of a larger 
research project to study attitudes of discharged patients towards professional care-givers and lay care-givers (Boderie and Sijstermans, 1986). Study VI took place as part of a research project 'Changes in Self-Care in relation to Coronary Artery Bypass Surgery' funded by the Dutch Heart Foundation. Purpose of this longitudinal study was to describe and explore changes in Self-Care needs and capabilities of patients having had coronary artery bypass surgery.

\subsection{Integrated Research Program}

\section{Purpose}

The purpose of the integrated research program in terms of meta-analysis of data of the combined seven studies was to test the internal consistency, equivalence and construct validity of the A.S.A.-scale. Internal consistency was tested by the following hypotheses.

H.1. Cronbach's alpha's of measurements with the A.S.A.-A scale will be $\geq$ .70 .

H.2. Item to total correlations of all 24 items of the A.S.A.-scale will be $\geq$ 30 .

H.3. $\overline{\mathrm{x}}$ item score of all 24 items of the group of $25 \%$ highest Total A.S.A.-A scores will be significantly higher than $\tilde{x}$ item score of these items of the group of $25 \%$ of the lowest Total A.S.A.-A scores.

H.4. The percentage of explained variance of the first component on the unrotated matrix of the factor-analysis of the A.S.A.-A scale will be twice the percentage of explained variance of the second component.

H.5. Item to factor correlations (loadings) of all 24 items on the first extracted component of the unrotated matrix of the factor-analysis of the A.S.A.-A scale will be $\geq .35$.

Equivalence was tested by the following two hypotheses.

H.6. Subjects' Total A.S.A.-A scores will be significantly positively $(r>.30$ $<$.70) correlated to these subjects' Total A.S.A.-B scores given by significant others.

H.7. Patients" Total A.S.A.-A scores will be significantly positively ( $r>.30$ $<$.70) correlated to these patients' Total A.S.A.-B scores given by nurses, who take care of these patients on a regular basis.

Construct validity was tested by the following hypotheses.

H.14. Subjects' Total A.S.A.-A scores will be significantly positively and weakly to moderately $(r \leq .40)$ correlated to General Assessment of Self-Care capability scores.

H.15. Total A.S.A.-A scores of subjects older than 65 years will be significantly negatively correlated with their age.

Construct validity was also explored by evaluating to what extent Power Components of Self-Care Agency are reflected in the factor structure of the factoranalysis of the A.S.A.-A scores. 


\section{Subjects}

Combined data of studies I until VII include A.S.A.-A scores of 676 subjects. These subjects completed a total of 908 A.S.A.-A measurements. The majority of the subjects were females $(n=409)$. Subjects mean age was 51.97 year S.D. 24.54. The youngest study participant was 18 and the oldest 97 . Of all subjects $35 \%$ were married and $27 \%$ lived alone. At the time of the interview 383 subjects lived at home, 30 in a service flat, 30 in a personalized care facility, 25 in a rehabilitation center, 40 in a nursing home and 167 stayed in an acute care hospital. The great majority $(n=606)$ lived in the southern part of the Netherlands, province of Limburg. Twenty seven percent lived in rural suburban area's, $38 \%$ in small cities and $35 \%$ in large cities. As far as subjects last occupational status $21 \%$ were blue collar workers, $22 \%$ white collar workers, $4 \%$ professionals, $19 \%$ housewifes, and $34 \%$ had student status. Subjects mean years of education was 10.97 years S.D. 3.47 with extremes of 3 and 22 years. The majority of the subjects $(n=383)$ had patient status and $52 \%$ had made use of treatment of a medical specialist half a year prior to data-collection. The most frequent first medical diagnosis of patients was disease of the circulatory system $(n=142)$ followed by diseases of the musculo-sceletal system $(n=71)$. Twentyone subjects had a disease of the nervous system, 18 of the respiratory system, while 13 patients' first diagnosis was classified as neoplasms. The rest of the patients had endocrinological diseases $(n=11)$, diseases of the digestive system $(n=10)$, of the genital-urological system $(n=8)$, infections $(n=2)$ and skin diseases $(n=2)$.

Data of the combined studies include A.S.A.-B scores of 219 significant others. They completed a total of 325 A.S.A.-B measurements. Significant others relations to A.S.A.-A scoring subjects were as follows: 135 partners, 46 children, 4 parents, 8 other family and 26 others. Significant others mean age was 55.85 S.D. 14.12 year. The majority were females $(n=118)$. As far as significant others last occupation 30\% were housewifes, $26 \%$ were blue collar workers, $36 \%$ white collar workers and $8 \%$ professionals. Significant others mean years of education was 9.58 years S.D. 3.77.

Data of the combined studies include 211 A.S.A.-B scores completed by nurses. The great majority of A.S.A.-B scales were completed by female nurses $(n=179)$. Nurses" mean age was 25.54 years with a S.D. of 5.84 . The youngest nurse was 20 and the oldest was 59 years old. Half of them were Registered Nurses and half of them were Licenced Practical Nurses. Ten percent of the R.N.'s were prepared at the baccalaureate level. Sixty percent of the nurses had a working experience of 5 years or more, while 13 percent had less than one year of working experience as a nurse. In nearly all $(92 \%)$ cases patient contact was on a daily basis. 


\section{Data-analysis Procedure}

Hypotheses 1 was tested by combining all A.S.A.-A scores of first measurements of the studies I till VII and by calculating Cronbach's alpha's for this total sample, for the subsample of predominantly older subjects with health problems of studies I till VI, for the subsample of predominantly younger subjects without health problems of study VII and for all studies I till VI separately. Hypotheses 2 was tested by calculating corrected item to total correlations for the individual 24 items of the A.S.A.-A scale based on combined data of studies I till VII, of studies I till VI and of study VII. For these three samples 25 percent of the highest and lowest Total A.S.A.-A scores were identified. Differences in $\overline{\mathrm{x}}$ item scores between these two groups of high and low Total A.S.A.-A scores were tested using $t$-test for independent samples (H.3). For the total sample of studies I till VII, for the combined data of studies I till VI and for the sample of study VII a factor-analysis was run on A.S.A.-A scores. The unrotated matrix was analysed for the percentage of the explained item variance of the first and following factors (H.4). Item to factor correlations (loadings) on the first component were also analysed (H.5.). Equivalence was tested by analysing the combined scores of studies I, II, III, IV and VI. Pearson"s correlation coefficients were calculated on the total sample for Total A.S.A.-A scores with significant others' Total A.S.A.-B scores (H.6) and with the nurses' Total A.S.A.-B scores (H.7).

Construct validity in terms of demonstration of convergence was tested by analysing the combined scores of studies I, II, IV, V and VII. Pearson's correlation coefficient was calculated between Total A.S.A.-A scores and General Assessment of Self-Care capability scores. (H.14) Construct validity in terms of testing a relationship with age (H.21) was done by combining data from studies I till VI. For the purpose of construct validity the structure of the extracted components of the rotated matrix of factor analysis was compared with the power components of Self-Care Agency. This was done for the total sample of studies I till VII, the sample consisting of data from studies I till VI and the sample of study VII separately.

\subsection{Study I: Difference in Self-Care Agency within an Elderly Population (ELPO)}

\section{Purpose}

The specific purpose of this study was to test for equivalence, short time interval stability and construct validity.

Equivalence was tested by the following hypotheses.

H.6. Total A.S.A.-A scores of elderly living independently in the community will be significantly positively $(r>.30<.70)$ correlated to these 
elderly Total A.S.A.-A scores given by their significant others.

H.7. Nursing home patients" Total A.S.A.-A scores will be significantly positively $(r>.30<.70)$ correlated to these patients' Total A.S.A.-B scores given by nurses, who take care of these patients on a regular basils.

Short time stability was tested by.

H.8. Total A.S.A.-A scores of elderly living independently in the community will be significantly positively and strongly $(r \geq .70)$ correlated when measured with a six weeks time-interval in which no major critical life events are experienced by them.

Construct validity was tested by use of known group technique. The following hypotheses were tested.

H.10. Total A.S.A.-A score of Elderly living independently in the community will be significantly higher than $\bar{x}$ Total A.S.A.-A score of Service-Flat residents.

H.11. $\overline{\mathrm{x}}$ Total A.S.A.-A score of Service-Flat residents will be significantly higher than $\overline{\mathrm{x}}$ Total A.S.A.-A score of Personalized Care Facility Residents.

H.12. Total A.S.A.-A score of Personalized Care Facility Residents will be significantly higher than $\overline{\mathrm{x}}$ Total A.S.A.-A score of Nursing Home residents.

Design

A partly cross-sectional and partly longitudinal correlational design was used. The A.S.A.-self-appraisal scale was administered to a sample of 40 randomly selected patients of a Nursing Home, 30 residents of a Personalized Care Facility, 30 residents of a Service-Flat. Furthermore 40 randomly selected subjects, who lived independently in the adjacent community were asked to rate their Self-Care Agency. Self-Care Agency of each nursing home patient was also appraised with the A.S. A.-B by two independent nurse raters $(n=80)$. Thirty-seven independently living subjects were also rated for their Self-Care Agency with A.S.A.-B by a significant other. Three subjects could not identify a significant other. After a period of 6 weeks the independently living elderly were asked to rate their Self-Care Agency again. For an overview of the total design see figure 4.1.

\section{Subjects}

Selection-criteria for the four groups of elderly were 65 years of age or older, no serious psycho-geriatric pathology, ability to answer questions and informed consent. The nursing home patients were selected from all units of the nursing home with the exeption of units for psycho-geriatric patients. Selection took place with a random digit's table. Based on selection criteria fourty-three 


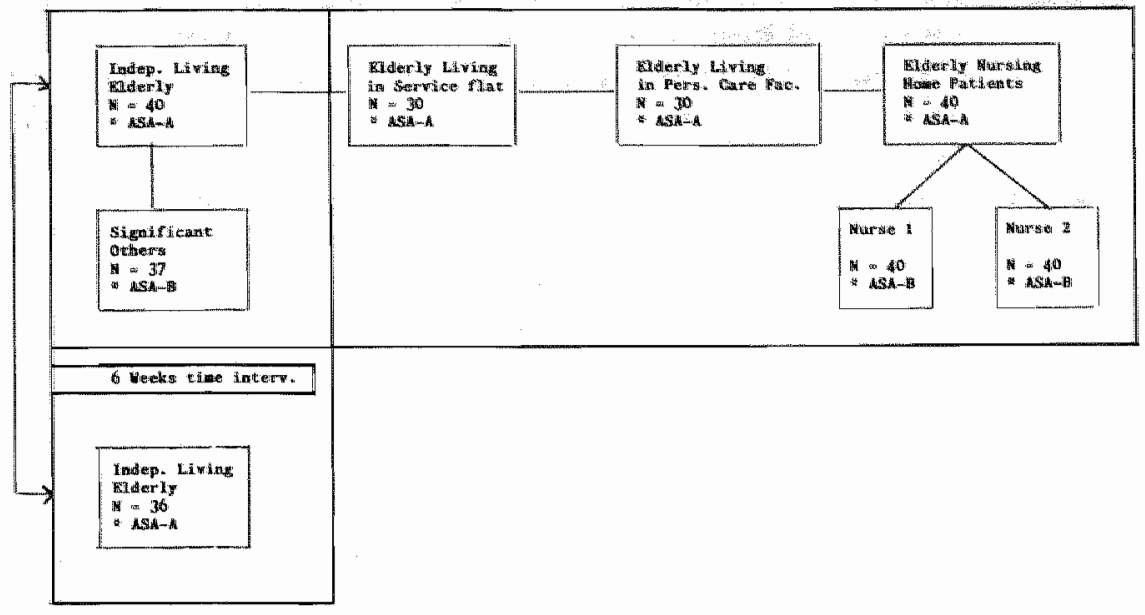

individuals in the Personalized Care Facility were eligable to participate. Thirty out of them gave their consent. All seven units of the facility were represented. In the Service-Flat a proportional stratified sample was drawn to guarantee equal representation of each corridor of the Service-Flat. A substantial amount of subjects drawn were not willing to participate. In a second round residents of the Service-Flat were randomly approached until the total number of 30 subjects was reached. Both the Nursing Home and the Personalized Care Facility with the adjacent service flat were located in a large city in the southern part of the Netherlands. The elderly living independently in the community were selected from three districts of the same city. These districts had the highest percentage of non-institutionalized citizen of 65 years or older. The percentages varied from 16 to 24 . A random sample was drawn out of all the streets in these districts. In every selected street house number 5 was approached for a possible study participant. In this way fourty elderly were recruted.

A total number of 140 elderly participated in this study. As far as possible the groups were matched for sex, age and socio-economic status. Twenty eight males and 112 females participated in the study. The mean age of these elderly was 80.4 years with a range from 65 to 97 . Fourty-nine were blue collar workers, twenty-seven white collar workers and three professionals. Fifty-nine participants were housewifes. For the total sample the mean years of education was 6.95 years with a range from 5 to 15 years. Thirty-seven elderly in the community identified a significant other able and willing to participate. Seventeen of them were partners, eleven were children and nine others. Thirteen were malle and twenty-four female. Their mean age was 63.78 years. In the nursing home 
every patient"s Self-Care Agency was rated by two nurses who cared for the patient for at least four previous weeks. No student-nurses were included in the study. The mean age of the nurses was 28.4 years with a range from 20 to 40 years. Out of the total of 80 seven ratings were done by male nurses and the rest by female nurses. Ten ratings were done by Registered Nurses (R.N.'s) and seventy by Licensed Practical Nurses (L.P.N.'s). In seven cases the nurse had less than a year working experience, in seventeen cases in between 1 and 5 years and in most cases $(n=56)$ the nurse had more than five years of working experience.

\section{Procedure}

Nursing Home patients and Personalized Care Facility residents were approached through headnurses to inform them about the study and to ask for consent. Subjects were interviewed by master's students in nursing science and a research assistant using a structured questionnaire. Data were collected on Self-Care Agency using the A.S.A.-A scale, General Assessment of Self-Care capability, age, sex, last occupation using the Dutch Job Classification System (Westerlaak van, Kropman and Collaris, 1975), marital state and number of years of formal education starting with the first year of primary school. Most interviews were done in local dialect. Interview time varied from 15 minutes to two hours (Schrijnemaekers, 1986). In the Personalized Care. Facility and the Service-Flat data were collected as part of another study (Boshouwers, 1987).

Significant others were also interviewed by a master's student using a structured questionnaire. Data were collected on the independently living elderly's Self-Care Agency using the A.S.A.-B scale (Cronbach's alpha $=.84$ ), Generall Assessment of the elderly's Self-Care capability, the significant other's age, sex and kind of relationship with the elderly. Interview time ranged from 15 minutes to one hour and a half. Most interviews were done in dialect. In the Nursing Home two nurses were asked to complete a personally delivered questionnaire independently for each participating patient. In most cases this was done in presence of the researcher. Data were collected on patient's Self-Care Agency using the A.S.A.-B scale (Cronbach's alpha $=.90$, interrater-reliability $r=.69, p<.000$ ), General Assessment of Self-Care capability, nurses's age, sex, kind of nursing diploma and total working experience. Average completion time was $\mathbf{8}$ minutes.

\subsection{Study II: Self-Care Agency, A.D.L. and Social Support of Reumatoid Arthritis Patients at home (RAP)}

\section{Purpose}

The specific purpose of this study was to test for equivalence and construct 
validity. Equivalence was tested by the following hypotheses.

H.6. Reumatoid Arthritis Patients' Total A.S.A.-A scores will be significantly positively $(r>.30<.70)$ correlated to these patients' Total A.S.A.-B scores given by their significant others.

H.7. Reumatoid Arthritis Patients' Total A.S.A.-A scores will be significantly positively $(r>.30<.70)$ correlated to these patients" Total A.S.A.-B scores given by their community nurses.

Construct validity in terms of demonstration of convergence was tested by the following hypothesis:

H.14. Reumatoid Arthritis Patients' Total A.S.A.-A scores will be significantly positively correlated to their General Assessment of Self-Care capability scores.

Construct validation in terms of demonstration of discriminability was tested by the following hypothesis.

H.15. Reumatoid Arthritis Patients' Total A.S.A.-A scores will not be significantly correlated with Total A.D.L. Index scores.

Construct validity in terms of examination of theoretical relationships was tested by the following hypothesis.

H.19. Reumatoid Arthritis Patients' Total A.S.A.-A scores will be positively correlated to Total Functional and total Network N.S.S.Q.-scores.

\section{Design}

A cross-sectional correlational design was used. Fourty-four non-hospitalized patients with the medical diagnosis Reumatoid Arthritis were interviewed in their homes. Data were collected in respect to their Self-Care Agency and their Social Support System. Thirty-nine significant others rated patient's SelfCare Agency and A.D.L. Community nurses of twenty-one patients also appraised patient's Self-Care Agency and A.D.L. For an overview of the total design see figure 4.2 .

\section{Subjects}

Selection criteria for the Reumatoid Arthritis Patients were age of 50 years or older, no other serious medical problems, ability to communicate in Dutch and informed consent. Subjects were randomly selected from district nurses' patient-lists. Nurses of three health-districts of a provincial Visiting Nurse Association in the Western part of the Netherlands cooperated in the study. The health districts included a large metropolitan area, several suburban area's and a rural area. A total of seventy-three patients were initially approached for participation. Fourty-eight out of them consented. Due to unexpected hospitalization and death the realized sample consisted of 44 patients. The average time since subjects were first diagnosed as Reumatoid Arthritic Patient was 19.3 years with extremes of 1 and 50 years. The mean age of the patients was 


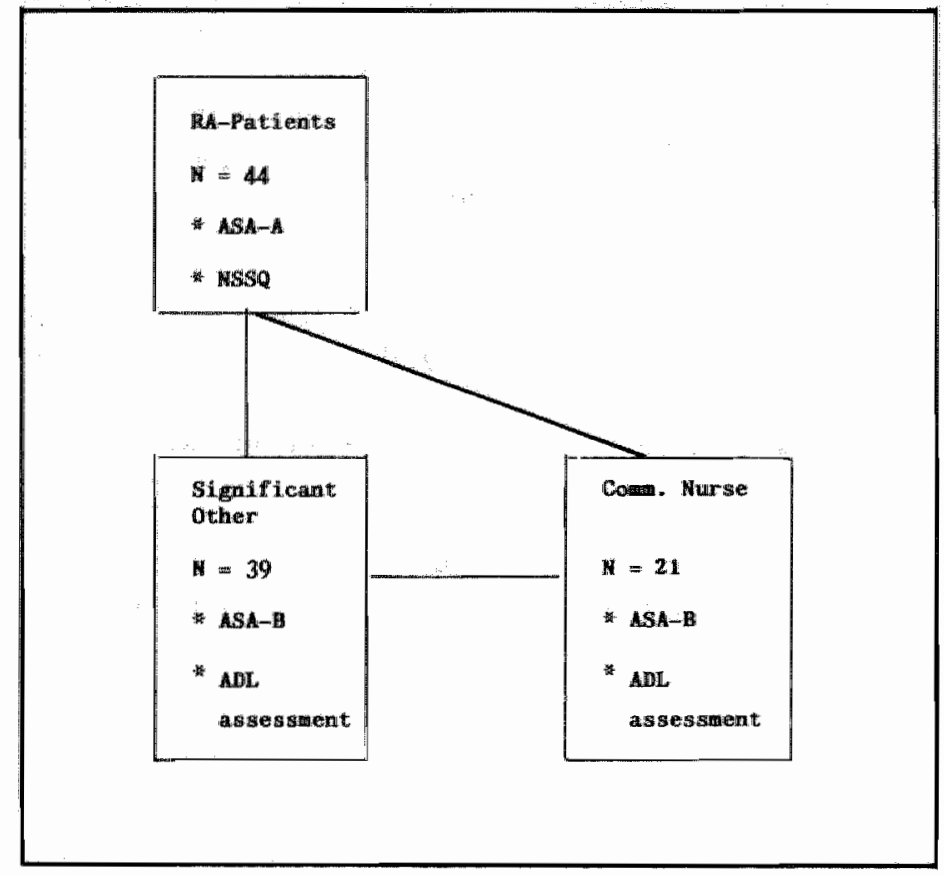

62.93 years with a SD of 6.86 . Thirty-five patients were females and 30 were married. Thirteen subjects lived alone. Fourteen patients lived in a rural suburban area and the rest in cities. The mean years of education was 9.5. Nine patients were blue collar workers, 16 low level white collar workers and the rest were higher classified workers. Six patients were housewifes. Out of the total 28 patients made use of housekeeping service and 23 of community nurses service.

Two of the 23 community nurses were not able to appraise patients' Self-Care Agency due to infrequent contacts. Mean age of the remaining 21 nurses was 35.14 year. All were female. The majority had a working experience of more than 5 years. Sixteen were prepared at the baccalaureate level (M.G.Z. or H.B.O.-V). In 5 cases there was a daily nurse-patient contact, in 10 cases a weekly and in the remainder a less frequent contact. Sixteen nurses knew the patient at least 3 years. Significant others were recruted by asking patients to identify a person with good knowledge of their daily functioning. In 39 cases a significant other was able and willing to participate. In 26 cases this person was a spouse, 6 were children and 7 others. Significant others' mean age was 57.06 year. Nineteen were males, nine were blue collar workers, 15 low white collars 
and the rest higher classified workers. Significant others had a mean of 10.33 years of education.

\section{Instruments}

Activities of Daily Living were measured by Katz" modified A.D.L. Index (Katz, 1976). This observational instrument was developed by Katz et al. (1963) to measure A.D.L. as biological and psychosocial function in the elderly and the chronically ill. The included functions are bathing, dressing, toilleting, transfer, continence and feeding. The rater judges the level of independence of the patient for each function as requiring no assistance, limited assistance and extensive assistance. The individual items are combined using a scale of ordered profiles of levels of dependence, 0 indicating total functional independence and 6 total functional dependence. Interrater reliability is reported as more than $95 \%$ interrater agreement. Statistically significant correlations with other measures of overall functional outcome indicate validity of the instrument. The instrument has been widely used also with Reumatoid Arthritis Patients at home (Katz et all. 1976, 1979). A version of the instrument translated into Dutch was available. In this study internal consistency of significant others' and nurses' A.D.L. ratings were .84 and .85 .

Social Support was measured by the Norbeck Social Support Questionnaire (Norbeck, 1981). The N.S.S.Q. measures perceived Social Support available to the individual. Major components are positive affect, affirmation and symbolic and material aid. Respondents are asked to list significant persons in their life up to a maximum of 20 . Each network member is rated on a 5 point Likert scale using 9 items. These items deal with functional properties (affect, affirmation and aid), with network properties (size, stability and availability and with recent losses. A Total Functional, a Total Network and a Total Loss subscale score can be calculated. Reported test-retest correlational coefficients ranged from .85 to .92 for a one week time interval. Internal consistency for the subscales ranged from .54 to .96 . Strong positive correlations were found between the Functional and Network subscale while no significant correlations were established with Total Loss. The instrument is reported to be free from significant variance in individual social desirability response bias. Additional work is needed to establish construct validity. The instrument can be selfadministered but due to arthritic related writing problems an interview form was used. The original instrument was translated into Dutch. Comprehensability and clearness of the translated version of the instrument was reviewed by five practicing nurses. In this study internal consistency of the N.S.S.Q. measurement was .91 .

\section{Procedure}

Patients were first contacted by mail followed by a phone call and later by a 
home visit. All patients were interviewed by the same master's student in nursing science. A structured questionnaire was used to collect the following data. Self-Care Agency using the A.S.A.-A scale, Social Support using the N.S.S.Q., General Assessment of Self-Care capability, duration of illness, sex, age, marital state, last occupation, number of years of formal education, degree of urbanisation of place of living using the C.B.S. classification (C.B.S., 1980) frequency and length of community nurses' service, and frequency of housekeeping service. Interview time ranged from $\mathbf{4 5}$ minutes to more than two hours due to patients need for communication (Schiltmans, 1987).

Significant others were interviewed by the same master's student using a structured questionnaire. Data were collected on patients' Self-Care Agency with the A.S.A.-B scale (alpha = .74). On patients' Activities of Daily Living with Katz' A.D.L.-index, on significant others' relation to the patient, their age, sex, last occupation and years of formal education. Interview time ranged from 10 to 30 minutes. Community nurses were interviewed by phone. Data were collected on patients' Self-Care Agency with the A.S.A.-B scale (alpha = .84), on patients' Activities of Daily Living with the A.D.L. Index, on General Assessment of patients' Self-Care capability, frequency and length of nursepatient contact and nurse's sex, age, basic nursing education and working experience. Average interview time was 15 minutes.

\subsection{Study III: Self-Care Agency of Institutionalized Rehabilitation Patients measured by E.S.C.A. and A.S.A. (REHAB)}

\section{Purpose}

The specific purpose of this study was to test for equivalence and construct validity. Equivalence was tested in this study by the following hypotheses.

H.6. Rehabilitation Patients' Total A.S.A.-A scores will be significantly positively ( $r>.30<.70$ ) correllated to these patients' Total A.S.A.-B scores given by their significant others.

H.7. Rehābilitation Patients' Total A.S.A.-A scores will be significantly positively $(r>.30<.70)$ correlated to these patients ${ }^{9}$ Total A.S.A.-B scores given by nurses who take care of these patients on a regular basis. Construct validity i.c. demonstration of convergence was tested by the following hypothesis.

H.13. Rehabilitation Patients' Total A.S.A.-A scores will be significantly positively and moderately $(r \geq .40)$ correlated to their Totall Short Version ESCAS-scores.

\section{Design}

A cross-sectional correlational design was used. Twenty-five patients institutionalized in a rehabilitation center were asked to appraise their Self-Care 
Agency both by means of the A.S.A.-A and the E.S.C.A.-scale. Twenty-three significant others rated patients' Self-Care Agency with the A.S.A.-B scale. Self-Care Agency of each rehabilitation patient was also appraised with the A.S.A.-B by two independent nurse raters $(n=50)$. For an overview of the total design see figure 4.3 .

Fig. 4.3. DESIGN Study III: REHAB

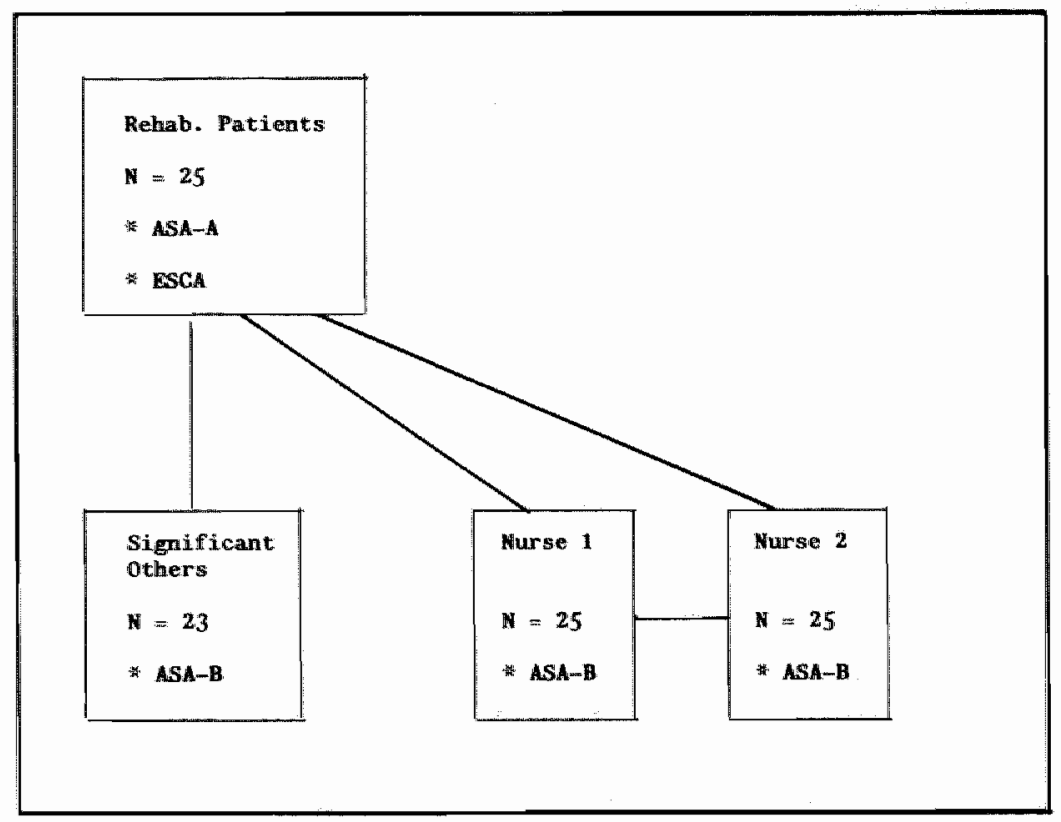

\section{Subjects}

Subjects were recruted in a rehabilitation center in the eastern part of the Netherlands. Total capacity of the center was 143 beds with 75 beds for adults and the rest for children. Only adults were included in this study. Selection criteria for participation were age of 18 year or older, length of stay in the center prior to the interview of one week or longer, ability to understand and answer questions, ability to concentrate for at least half an hour and informed consent. Based on the first four selection criteria thirty patients were eligable. Four patients did not consent and one patient turned out not to meet selection criteria afterwards. Thus the total sample consisted of 25 patients. The mean age of the patients was 53.44 year while the youngest patient was 20 and the oldest 84 . Seven of them were male and 18 female. Eleven patients were married. Fourteen patients suffered from diseases of the nervous system and 10 
from diseases of the musculoskeletal system. Half of the patients were hospitalized in between one and four months. Six patients were blue collar workers, 13 white collar workers, 5 housewife and one was a professional. For the total sample the mean years of education was 9.28 year with a range from 4 to 17 .

All patients were able to identify a significant other but in two cases due to practical circumstances an interview could not be scheduled. Thus a total of 23 significant others participated in the study. Eight of them were spouses, 3 children, 4 parents and the rest others. Every patient's Self-Care Agency was. also rated by two independent nurse raters who cared for the patient at least one week. No student nurses were included in the study. The mean age of the nurses was 26,4 years with a range from 21 to 43 . Six of the nurses were male. The majority were Licenced Practical Nurses and the rest Registered Nurses, two of them baccalaureate prepared. The great majority worked in the rehabilitation center for more than one year.

\section{Instruments}

The Exercise of Self-Care Agency Scale E.S.C.A.S. is a 43 item Likert type self-appraisal instrument (Kearney and Fleischer, 1979). The theoretical scores range from 0 to 172 indicating the highest possible degree of Exercise of Self-Care Agency. Test-retest reliability coefficients were $.77, .81$ and .84 . In terms of content validity 16 items reflect the power to perform Self-Care operations (numbers $1,4,8,11,13,15,17,19,27,29,35,36,37,39,40,42$ ), while 20 items reflect foundational human dispositions and capabilities. The original instrument was translated into Dutch. Comprehensability and clearness of the translated version was reviewed by 5 persons. In this study Cronbach's alpha for the E.S.C.A. measurement was 72 .

In this study internal consistency of significant others' A.S.A.-B measurement was .69. Cronbach's alpha of the A.S.A.-B measurement of the two groups of nurses were .86 and .75 . The interrater reliability for A.S.A.-B measurement between the two groups of nurses was $\mathrm{r}=.71, \mathrm{p}<.000$.

\section{Procedure}

The research protocol was introduced to headnurses and staffnurses by the Director of Nursing Service and a master's student in nursing science. Headnurses were asked to select patients with the master's student based on established criteria. Selected patients were personally contacted. After informed consent an interview was scheduled. All interviews were done by the master's student using a structured questionnaire. Data were collected on Self-Care Agency using A.S.A.-A and E.S.C.A. Instrument order was randomly established for each patient. Other collected data were patient's age, sex, marital state, medical diagnosis classified according I.C.D. 9, number of years of 
formal education and last occupation. Interview time ranged from 30 to 45 minutes (Kalter, 1987).

Significant others were identified by the patients and interviewed by the master's student at home or in the rehabilitation center during visiting hours. Using a structured questionnaire data were collected on patients" Self-Care Agency using A.S.A.-B, and the nature of significant others' relation to the patient. Interview time ranged from 15 to 30 minutes. For each patient two nurses were asked to complete a personally delivered questionnaire independently. This was done in presence of the master's student. Data were collected on patients' Self-Care Agency with A.S.A.-B, nurses' age, sex, basic nursing education, time worked as a nurse in general and in the center. Completion of each questionnaire took about 10 minutes.

\subsection{Study IV: Appraisal of Acute Care Hospital Patients' Self-Care Agency by Nurses, Patients and Significant Others (HOP)}

\section{Purpose}

The specific purpose of this study was to test for equivalence and construct validity. Equivalence was tested by the following hypotheses.

H.6. Acute Care Hospital Patients" Total A.S.A.-A scores will be significantly $(r>.30<.70)$ correlated to these patients' Total A.S.A.-B scores given by their significant others.

H.7. Acute Care Hospital Patients' Total A.S.A.-A scores will be significantly positively $(r>.30<.70)$ correlated to these patients' Total A.S.A.-B scores given by nurses, who take care of these patients on a regular basis.

Construct validity in terms of demonstration of convergence was tested by the following hypothesis.

H.14. Acute Care Hospital Patients' Total A.S.A.-A scores will be significantly positively correlated to their General Assessment of Self-Care capability scores.

\section{Design}

A cross-sectional correlational design was used. The A.S.A.-B scale was administered to a randomly selected sample of sixty nurses of an Acute Care Hospital. The A.S.A.-A to a sample of sixty patients under care of these nurses. The A.S.A.-B scale was also administered to a sample of sixty significant others belonging to the patients. This sample was added as a control group for the nurses. For an overview of the design see figure 4.4 . 


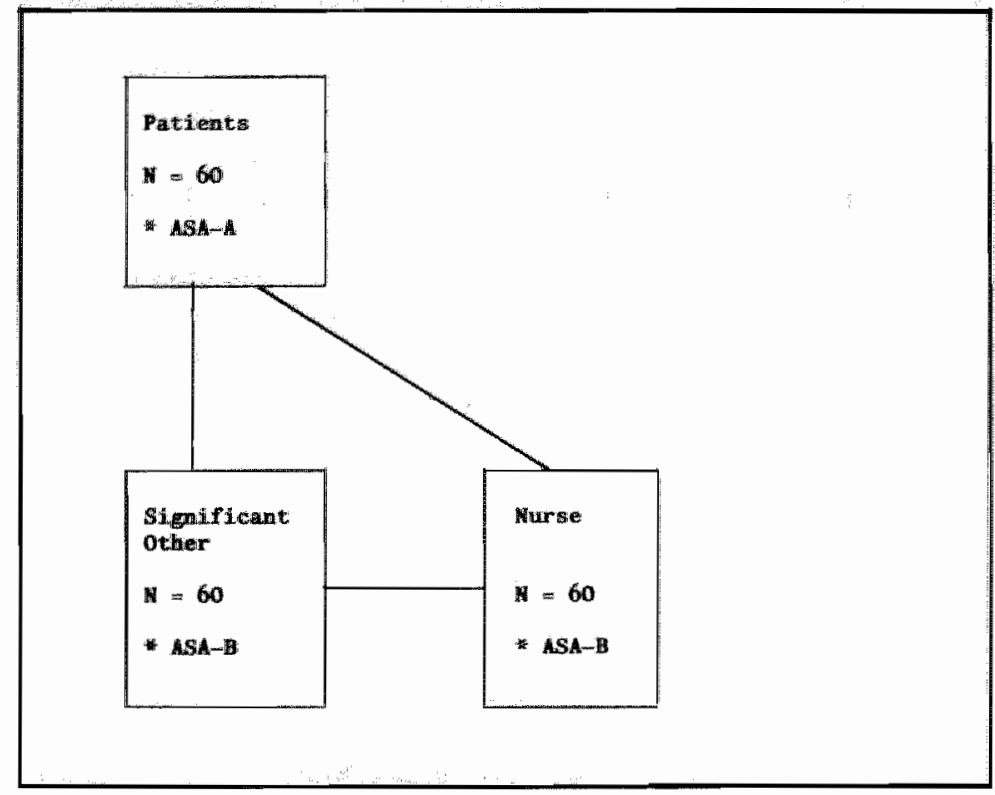

Subjects

Selection criteria for patients were age of 18 years or older, admission to the in-patient department of the hospital, ability to communicate in Dutch, ability to identify a significant other who can and will participate and informed consent. Patients mean age was 59.28 year with a range of 25 and 85 . Twenty-four were males. Fourty-three patients were married. Patients had a variety of medical diagnoses. Most patients (12) suffered from neoplasms followed by 10 patients with diseases of the circulatory system. Eight patients had diseases of the respiratory system and the same number had diseases of the musculo skeletal system. Seven patients had diseases of the tractus digestivus and six of the tractus geniuritalis. The rest had endocrinological disorders or diseases of the nerwous system. All patients except of one lived in the province of Limburg, 32 in rural suburban area's and 28 in cities. Nineteen patients were blue collar workers, 18 white collar workers, 3 professionals and 20 housewife. The mean years of formal education was 9.47 years with a minimum of 4 and a maximum of 18 years.

Selection criteria for the nurses were the following. The nurse is actively providing direct patient care in the hospital's in-patient department. Excluded were nurses of pediatric wards due to inadequacy of the A.S.A.-scale for 
children, nurses of intensive and coronary care, heamodialysis and obstetrics all for practical reasons. The nurse is a Registered Nurse or a Licenced Practical Nurse. Excluded were student nurses and nurse aides. The nurse must be able to identify a patient whom she or he knows well and who would adhere to sample selection criteria. The last criterion was informed consent. A random sample of 100 nurses was drawn from the total nursing workforce of 720 individuals of a large Acute Care Hospital in the Southern part of the Netherlands. Sample selection was carried out by taking every fifth card from the central nursing personnel data file. Nurses meam age was $28: 11$ years SD 5.5. Fifteen of them were male and 45 female. All subjects were Registered Nurses and one was baccalaureate prepared. Eleven nurses had less than two months working experience. Thirty-four had worked as an R.N. for more than five years. The mean length of nurse patient contact was 14 days. Selection criteria for significant others were age of 18 years or older, ability to communicate in Dutch and informed consent. Most of them (30) were spouses, 20 were children and the rest others. Significant others mean age was 50.18 year (SD 14.15). Twenty-five were males. Twenty were blue collar workers, an equal number white collar workers, 3 professionals and 16 housewifes.

\section{Procedure}

Headnurses were personally contacted and asked to give a letter of introduction to the selected nurses. A letter of consent of the director of nursing service and of the general hospital management as well as a summary of the research protocol was added. Nurses were contacted by a master's student in nursing science by phone. The nurse was asked for consent and to identify a patient. Next the patient was contacted, given a summary of the study, a letter of consent of the general hospital management and some verbal explanation by the master's student. Consent was asked. At the same time the patient was asked to identify a significant other and the time he or she would visit the patient. Significant others were asked in the same way to participate. If consent was obtained of a complete triad arrangements were made for data collection preferably on the same day but independently for the three different subjects involved (Courtens, 1987).

Patients were interviewed using a structured questionnaire by the master's student in nursing science. Data were collected on Self-Care Agency with A.S.A.-A scale, General Assessment of their Self-Care capability, age, sex, marital state, medical diagnosis, place of living, last occupation and years of formal education. Length of interview time varied from 10 to 45 minutes. Nurses were asked to complete a self administered questionnaire. Data were collected on patients Self-Care Agency with A.S.A.-B (Cronbach's alpha = .78) nurses General Assessment of patients Self-Care capability, nurses sex, age, basic nursing education, length of professional working experience and 
length of nurse patient contact. The average time to complete the questionnaire was 10 minutes. Significant others were again interviewed using a structured questionnaire by the same student. Data were collected on patients' Self-Care Agency with A.S.A.-A, General Assessment of patients Self-Care capability, significant others' relation to patient, significant others' age, sex and occupation. Interview time ranged from 10 to 45 minutes.

\subsection{Study V: Self-Care Agency and A.D.L. of Patients discharged from an Acute Care Hospital (DIHOP)}

\section{Purpose}

The specific purpose of this study was to test for construct validity. Construct validity in terms of demonstration of convergence was tested by the following hypothesis.

H.14. Discharged Acute Care Hospital patients' Total A.S.A.-A scores will be significantly positively correlated to their General Assessment of Self-Care capability scores.

Construct validation in terms of demonstration of discriminability was tested by the following hypothesis.

H.15. Discharged Acute Care Hospital patients' Total A.S.A.-A scores will not be significantly correlated with their A.D.L. scores.

\section{Design}

A cross-sectional correlational design was used. The A.S.A.-A self-appraisal scale was administered to a sample of 77 patients two weeks after discharge from an acute care hospital. A self-appraisal instrument to evaluate their A.D.L. was also administered. This design was part of a larger study. For the relevant part of the design for this study see figure 4.5 .

\section{Subjects}

Selection criteria for subjects were being discharged from an acute care hospital two weeks prior to data collection time, age between 55 and 80 years, living independently at home in the same city where the hospital was located and informed consent. The sample should also include 50 percent subjects receiving aftercare and 50 percent receiving no care after discharge. Aftercare was defined as receiving community nursing care or housekeeping, home maintenance assistance.

A timebound sampling technique was used. Based on particiption of 10 hospital wards, an average of 2 interviews a day and a data collection period of 3 months a sample of 100 subjects was projected. A total of 113 discharged patients were approached. Thirty-six patients, 21 females and 15 males refused to participate 


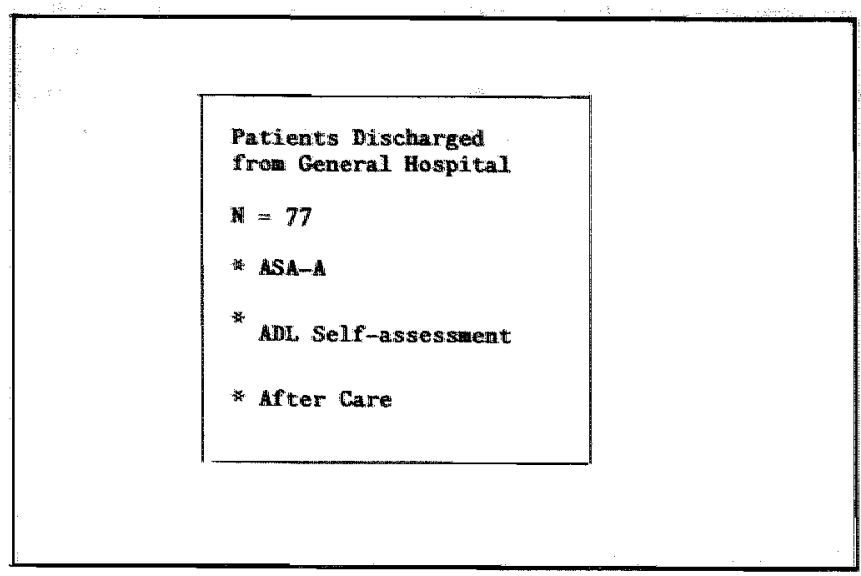

due to lack of interest, their physical condition and family pressure. Thus in the end a total sample of 77 patients was reached fifty of them without aftercare and only twenty-seven with aftercare. Patients mean age was 66.68 year SD 7.16. Fourty-one were females and fourty-nine were married. Twenty-eight patients lived alone. Eleven subjects received community nursing care and 17 home maintenance assistance.

\section{Instrument}

Activities of Daily Living were measured by an adapted version of the Physical A.D.L. component of the Multidimensional Functional Assessment Questionnaire (Pfeiffer, 1975). The following functions were measured bathing, dressing, eating, getting to the bathroom in time, transferring in and out bed, standing and walking. The responent was asked if he/she performed each activity independently, with some help or with complete help. A five week test-retest correlation coefficient $r=.82$ is reported for the original version of the Physical A.D.L. component of the M.F.A.Q. (Pfeiffer, 1975). In this study Cronbach's alpha was .60 for the adapted version. The item 'getting to the bathroom in time' was not included in the analysis for no variance at all was demonstrated for this item.

\section{Procedure}

Data-collection for the purpose of this study took place as part of a larger research-project to study attitudes of discharged patients towards professional care givers and lay care givers (Boderie \& Sijstermans, 1986). Headnurses of 10 non-specialty wards of a university medical center in the southern part of the Netherlands were asked to prepare a daily list of patients planned for discharge 
based on study selection criteria. Patients included in the list were visited before discharge. After informed consent a home visit was schedulled. Due to changes in discharge-time not all eligable patients could be approached. Data were collected by two master's students in nursing science. The A.S.A.-A scale was self-administered by patients while other data were collected by interview. Other data included patients General Assessment of Self-Care capability, their A.D.L., sex, age, marital state, use of community nursing service and home maintenance service. Data were collected September till December 1985.

\subsection{Study VI: Change in Patients" Self-Care Agency after Coronary Artery Bypass Surgery (CABS)}

\section{Purpose}

The purpose of this study was to test for equivalence, long-time stability, construct validity and the influence of certain measurement conditions.

Equivalence was tested by the following hypothesis.

H.6. C.A.B.S. Patients' Total A.S.A.-A scores will be significantly positively $(r>.30<.70)$ correlated to these patients' Total A.S.A.-B scores given by their significant others.

Long-time stability was tested by the following hypothesis.

H.9. C.A.B.S. Patients' six months post-operative Total A.S.A.-A scores will be significantly moderately and positively $(r \geq .40)$ correlated to their twelve months post-operative Total A.S.A.-A scores.

Construct walidity in terms of proposed relationships between Self-Care Agency and Self-Care and between Health State and Self-Care Agency was tested by the following hypotheses.

H.16. C.A.B.S.-patients' Total A.S.A.-A scores will be significantly positively correlated to their Total R.P.R.I.-scores.

H.17. C.A.B.S.-patients' 6 months post-operative $\tilde{\bar{x}}$ Total A.S.A.-A score will be significantly higher than the same patients' pre-operative $\bar{x}$ Totall A.S.A.-A score.

H.18. C.A.B.S.-patients' 12 months post-operative $\bar{x}$ Total A.S.A.-A score will be significantly higher than the same patients' pre-operative $\bar{x}$ Total A.S.A.-A score.

The effect of measurement conditions was tested by the following hypotheses.

H.22. C.A.B.S. Patients' six months post-operative Total A.S.A.-A scores will not be significantly correlated to these patients' six months postoperative Total scores on the adapted C. \& M. Social Desirability scale.

H.23. $\overline{\mathrm{x}}$ Total A.S.A.-A score of C.A.B.S.-patients to whom the scale is personally administered twelve months post-operative will not significantly differ from $\bar{x}$ Total A.S.A.-A score of fellow C.A.B.S.-patients to whom the scale is send by mail twelve months post-operative. 


\section{Design}

A longitudinal correlational design was used consisting of one pre-operative and two post-operative measurements. Post-operative measurements were at 6 and 12 months after surgery. The A.S.A.-A scalle was personally administered to Coronary Artery Bypass patients one or two days prior to surgery. At the same time patients' Universal Self-Care was measured using the Revised Patient Recovery Inventory. Spouses or other significant others were also asked to appraise patients' Self-Care Agency using the A.S.A.-B scale. Six months after surgery the A.S.A.-A scalle and the R.P.R.I. were personally administered to patients at their homes in combination with a social desirability scalle. Significant others were also asked again to appraise patients' Self-Care Agency with A.S.A.-B. At 12 months measurement time a randomly selected subgroup of patients received a mailed questionnaire which included A.S.A.-A and R.P.R.I. The remainder of the patients were visited again at home and personally administered A.S.A.-A and R.P.R.I. This somewhat indirect way of testing the effect of variation in measurement condition was chosen because of expected bias produced by the more direct way of administering the A.S.A.A in the two different ways to the same subjects at the same time. These patients significant others were also asked to appraise patients' Self-Care Agency. For an overview of the total design of this study see figure 4.6.

\section{Subjects}

Selection criteria for subjects were: admission for Coronary Artery Bypass Surgery, no psychiatric disorders, no pre-operative infection, Dutch born, ability to read and write Dutch and informed consent. Patients planned for C.A.B.S. in combination with valve-replacement and emergency surgery patients were excluded. The method of time bound sampling was used. Subjects were recruted from a University Hospital in the southern part of the Netherlands. During a 20 week period 152 patients were admitted for C.A.B. Surgery. A total of 123 persons met the outlined criteria. Sixteen persons refused to participate mostly for reasons of minimalizing tension during their hospital stay. Thus 107 subjects entered the study 81 men and 26 women. Sixty-one patients had had a myocardial infarcion prior to surgery. Eight patients were undergoing their second C.A.B.S. Patients mean age at the time of surgery was 60.46 year with a range from 42 to 77 years. Eighty patients were married. Fifty-nine patients lived in urban area's, fourty-nine in suburban rural area's. Thirty-six patients were blue collar workers in their last job, fourty-five white collar workers, twelve professional and fourteen housewife. Patients mean years of education was 9.59 year with a range from 3 to 22 . Two patients died from severe post-operative complications. Seven patients discontinued participation after surgery and 98 patients completed six month measurement. One patient died seven months after C.A.B. Surgery but one patient who refused at 


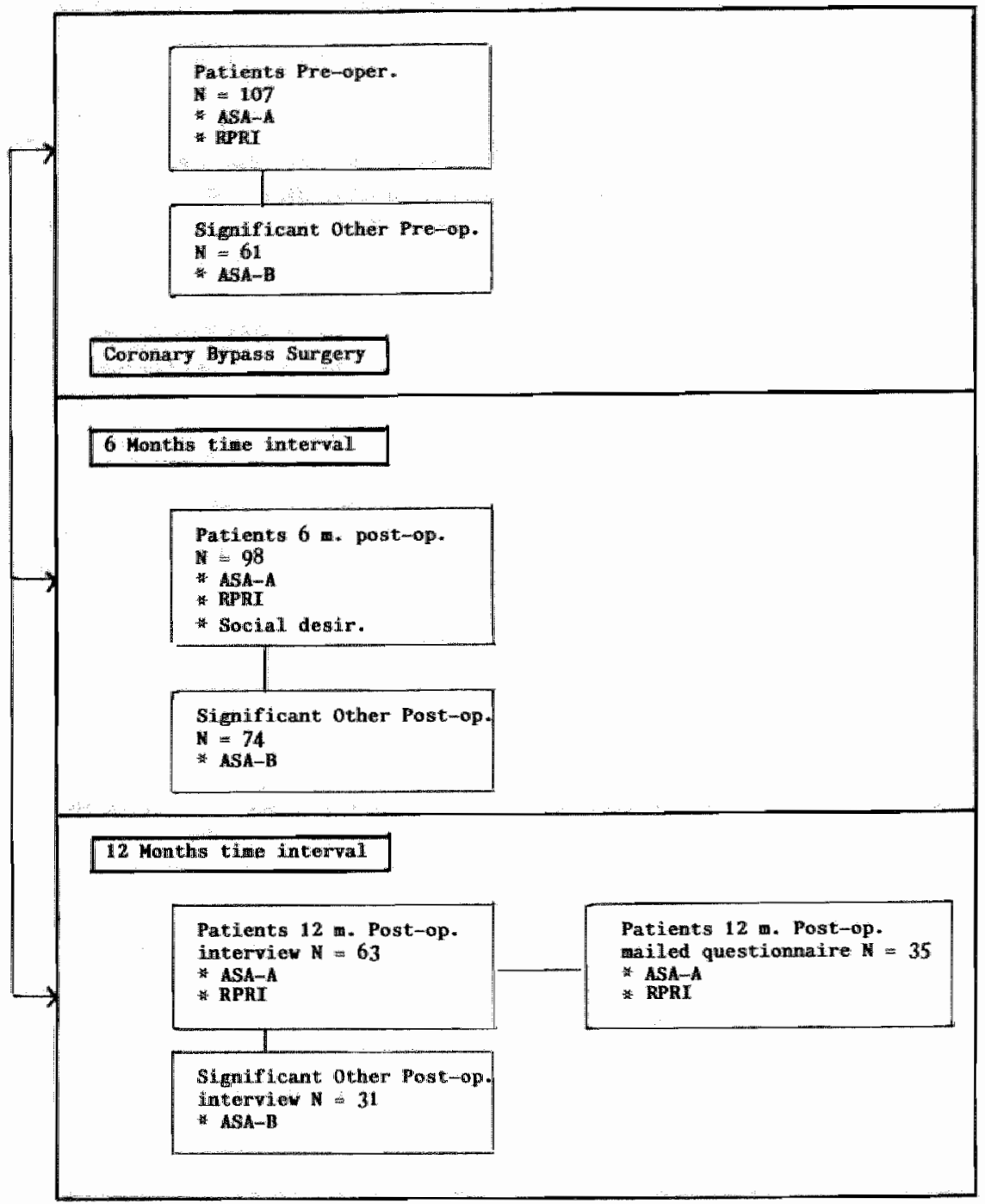

six months indicated willingness to participate in twelve months measurement. Thus a total of 98 patients completed the last phase of the study.

Prior to surgery 60 patients identified a significant other able and willing to participate. Fifty-one of them were spouses, six children and the rest had an 
other relation to the patient. Ten significant others were males. Significant others mean age was 55.89 year SD 8.42. Twenty-five significant others were housewife, nine were blue collar workers, fourteen white collar workers and one professional. Their mean years of education was 8.93 year ranging from 3 to 21 years.

\section{Instruments}

Self-Care in terms of the extent in which patients met their Universal SelfCare Requisites was measured by the Revised Patient Recovery Inwentory R.P.R.I. The Patient Recovery Inventory as developed by Eisler, Wolfer and Diers elicits patients' assessment of their condition. Pearson's correlation coefficients between P.R.I.-scores and nurses' ratings of these patients physical assessment ranged from $r=.67$ to $r=.69$ (Eisler et al., 1972). The Revised version of the R.P.I. included all aspects of the Universal Self-Care Requisites as stipulated by Orem. Patients are asked to rate on a ten point scale each need. High scores indicated that the need was optimally met. Summation of the scores gives a measure of adequacy of Self-Care a score of 0 meaning completely inadequate Self-Care and one of 110 the most adequate Self-Care. The instrument was translated into Dutch. Comprehensability and clearness of the translated version was reviewed by 3 persons. In this study Cronbach's alpha's of the R.P.R.I. ranged from .81 pre-operative to .79 six months post-operative and to .87 twelve months post-operative.

Social Desirability was measured by a shortened and adapted version of Crowne and Marlowe's C.M. Social Desirability Scale (Crowne and Marlowe, 1964). The original scale attempts to locate individuals who describe themselves in favorable, socially desirable terms in order to achieve approval of others. Items include two types of statements in a balanced way: culturally acceptable but probably untrue and half true but undesirably. The original scale consists of 33 items half keyed in the true and half in the false direction. Each response in the socially desirable direction is scored with one point. Theoretically total scale scores range from 0 (no social desirability) to 33 (highest social desirability). Internal consistency coefficient (Kuder Richardson 20) is reported to be .88. A test-retest correlation on a one-month interval with college students was .88 . Validity of the scale was supported by confirmation of several hypotheses in experimental settings although certain questions were raised about the theoretical foundations of the scale. Factor-analyses indicated the existence of two subscalles: Deny Bad Qualities and Claim Good Qualities. The first subscale appears to contribute most of the variance in correlations between the Total M.C. score and measures of other variablles (Robinson and Shaver, 1973). Hermans, Visser and others have used a translated version in several studies with Dutch populations including cardiac patients (Hermans, 1976; Visser, 1984). Based on factor analysis of study results the number of items was reduced 
to 11. Measuring technique was also adapted. In this version respondents were asked to indicate with a pencil a point on an uncallibrated $10 \mathrm{~cm}$ horizontal line to what extent they agreed with the statement as reflective of themselve. Indicated points were compared with a callibrated $10 \mathrm{~cm}$. line and given a score from 0 to 10. The theoretical range of the adapted M.C. scale was in between 0 and 110. Internal consistency of this version of the scale is reported as Cronbach's alpha $=.65$ (Visser, 1984). In this study Cronbach's alpha was .62.

\section{Procedure}

Data collection for the purpose of this study took place as part of the research project "Changes in Self-Care in relation to Coronary Artery Bypass Surgery'. At the pre-operative visit to the out-patient clinic (one week prior to surgery) or at the day of hospital admission (usually one day before surgery) patients were approached by one of the two researchers of the project. After explanation of purpose and procedure of the study patients were asked to participate and to sign a consent form. This was followed by first data collection. The A.S.A.-A was self-administered while other data were gathered by interview with a structured questionnaire. Data collected for the purpose of this study were patient's Self-Care with the R.P.R.I., patient's past cardiologic or cardiosurgical experience (prior m.i. or C.A.B.S.), patients sex, age, marital state, degree of urbanisation of place of living, last occupation and years of formal education. At the same time accompanying significant others were approached by the other researcher. After informed consent they were asked to appraise patients' Self-Care Agency with the A.S.A.-B scale (Cronbach's alpha = .71). Data were allso collected on significant other's relation to the patient, their sex, age, last occupation and years of formal education.

Six months after surgery subjects were contacted by phone to schedule a home visit. Both the A.S.A.-A and the Social Desirability scale were then self-administered. Completion of these instruments was separated in time by a ten minute interview. Patients" were interviewed on Self-Care using the R.P.R.I. and on other variables not relevant for the purpose of this study. The researcher also administered A.S.A.-B (Cronbach's alpha $=.79$ ) to significant others. The presence of the researcher guaranteed independent completion of A.S.A.-A and A.S.A.-B. All home visits were done by the same researcher.

Twelve months after surgery subjects again were contacted by phone. A random sample of 35 subjects was drawn. These subjects received a mailed questionnaire which included A.S.A.-A and R.P.R.I. In case of completion problems the possibility was offered to contact the researcher by phone. No one used this opportunity. The other subjects were visited again at home by the same researcher. A.S.A.-A was administered to subjects and A.S.A.-B (Cronbach's alpha $=.75)$ to significant others in presence of the researcher. Data on 
Self-Care were collected by interview using R.P.R.I. Data collection started January 1987 and was completed June 1988. All participating subjects received a small present at the end of the study.

\subsection{Study VII: Self-Care Agency and sex-stereotyped Self Image of Healthy College Students (COLS)}

\section{Purpose}

The specific purpose of this study was to test for construct validity. Construct validity in terms of demonstration of convergence was tested by the following hypothesis.

H.14. College Students Total A.S.A.-A scores will be significantly positively correlated to their General Assessment of Self-Care capability scores. Construct validation in terms of examination of the relationship between sexstereotyped self-image as measured by the G.R.A.S. Adjective Check-list and Self-Care Agency was tested by the following hypothesis.

H.20. College Students' Total A.S.A.-A scores will be significantly but weakly $(r< \pm 0.30)$ correlated to total G.R.A.S. scores.

\section{Design}

A cross-sectional correlational design was used. The A.S.A.-A self-appraisal scale was administered to a sample of School of Social Work College Students. At the same time the G.R.A.S. Adjective Check-list measuring sex-stereotyped self-image was administered. These instruments were also administered to a sample of School of Business College Students. For an overview of the design see figure 4.7. College students were chosen because of expected sample homogeneity in relation to possible confounding variables like age, health status, working experience e.a. College students were also relatively easy to approach collectively for participation in the study. School of business students were chosen, because traditionally this student population used to be predominantly male in the Netherlands. School of social work students were chosen because of expected different scores in sex-stereotyped self-image in relation to school of business student scores. Social work is more associated with qualities traditionally ascribed to females than business. Furthermore students sex is another critical variable. Thus the study was designed to compare four subgroups: male school of business students, female school of business students, female school of social work students and male school of social work students. For purpose of statistical analysis all groups needed a sufficiently large N. Projected was a total of 50 for each group. 


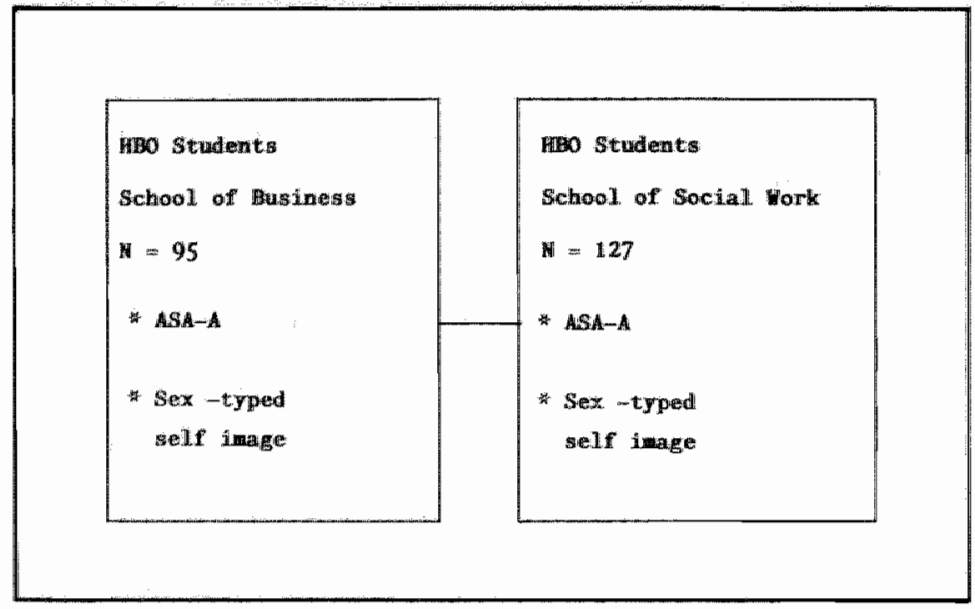

\section{Subjects}

Selection criteria for subjects were: age of 18 years or older, student of a school of business or a school of social work and informed consent. A conveniance sampling procedure was used. Subjects were recruted from one college of business and from one college of social work. Both colleges were located in the same city in the southern part of the Netherlands. Due to practical reasons in the school of business second and third year students were asked to participate and in the school of social work first and second year students. In the school of business all second and third year students were approached for participation. Out of them 95 consented, 47 males and 48 females. In the school of social work all approached first and second year students consented to participate $(n=128)$. The great majority $n=93$ were female. Thus the total sample consisted of 223 college students, 82 male and 141 female students. Students mean age was 20.67 year SD 1.97. Their mean years of formal education was 13.76 years SD 0.91. Sixty-seven lived alone in a one person unit. Seventeen students were married or had a comparable permanent relationship. Eighty four students lived in rural suburban area's, the rest in cities. The great majority $\mathrm{n}=198 \mathrm{had}$ no medial diagnosis. Five students had a disease of the respiratory system, four students a disease of the circulatory system, four had infections. One student had a neoplasm. Other diseases were classified under endocrinological disorders, disease of nervous system, of the digestive system, of the musculo-skeletal system, skin diseases and undiagnosed signs and symptoms. Twenty students had made use of treatment of a medical specialist during half a year prior to data collection. 


\section{Instrwments}

Sex-stereotyped self-image was measured with the Groningen Androgynie Schaal G.R.A.S. developed by de Graaf (De Graaf, 1984) and based on the Bem Sex Role Inventory (Bem, 1974; Bem e. a., 1976). The scalle contains two subscales: the M-scale with 29 masculine stereotype adjectives and the F-scale with 29 feminine stereotype adjectives. These 58 items were selected from an original list of 1204 personality adjectives. Based on perception scores of typical masculine and feminine qualities the adjectives were selected for the $\mathrm{M}$ - and F-scale. To hide the nature of the scale to respondents 24 sex-neutral adjectives were added. Respondents were asked to rate the adjectives as not at all descriptive of me, not descriptive of me, somewhat descriptive of me and completely descriptive of me. Scores ranged from 0 (not at all descriptive) to 3 (completely descriptive). Internal consistency of the $\mathrm{M}$ - and F-scale was reported as Cronbach's alpha $=.79$ and .86 In this study Cronbach's alpha's were respectively .84 and .82 . Subjects' scores on the $\mathbf{M}$ - and F-scale were combined to one score: the sex-stereotype value. This is the value of $T$ calculated as a result of statistically testing respondents difference in mean score on the $\mathrm{M}$ - and F-scale. Based on these stereotype values respondents self-images could be classified into four groups. Subjects with significant higher scores on masculine adjectives $(T(56)=-2.00, p<0.05)$ were classified as having a masculine stereotype self-image. The higher the negative t-value the more masculine stereotype the self-image. Subjects with significant higher scores on feminine adjectives $(T 56)=+2.00$, p. $<0.05$ ) were classified as having a feminime stereotype self-image. The higher the positive t-value the more feminine stereotype the self-image. Subjects with scores above the median of the $M$ - and F-scale but with no statistically significant difference between their mean $\mathrm{M}$ and $F$-scale were classified as having an androgyne (both masculine and feminine qualities) stereotype self-image. Subjects with scores below the median score on both M- and F- scale were classified as 'unclear". In de Graaf's Study with university students males could be most often classified as having a masculine-stereotype self-image and females as having an androgyne stereotype self-image.

\section{Procedure}

Directors of both schools were personally contacted, informed about studypurpose and data collection criteria and asked for cooperation. The director of the school of business informed all second and third year students about the study with a standard letter of introduction. He allowed students willing to participate to leave classes 15 minutes prior to lunch-time and organised a lecture hall for data collection. The director of the school of social work arranged one faculty teaching a class to all first and second year students and willing to ask students for participation at the end of lecture time. All students 
were willing to participate and to stay. Participating students were briefly informed about data collection procedure by a master's student in nursing science. All students received a questionnaire. Included were the A.S.A.-A scale and the G.R.A.S. Adjective Check List. Data were also collected on subjects General Assessment of Self-Care capability, subjects sex, age, years of formal education, housing situation, marital state, degree of urbanisation of place of living and health status (medical diagnosis and use of treatment of medical specialist). Questionnaires were completed in presence of and were collected by the master's student. Average completion time was twenty minutes. Data were collected in Februari and March 1988 (van Achterberg, 1988).

\subsection{Summary of hypotheses in relation to studies}

In order to give an overview of all reliability and validity aspects addressed in this research program as summary follows of all hypotheses described in chapter three in relation to the studies described in this chapter.

\section{Reliability}

Hypotheses

H.1. Cronbach's alpha's of measurements with the A.S.A.-A scale will be $\geq .70$

H.2. Item to total correlations of all 24 items of the A.S.A.-A scale will be $\geq .30$

H.3. $\quad \overrightarrow{\mathrm{x}}$ item score of all 24 items of the group of $25 \%$ highest Total A.S.A.-A scores will be significantly higher than $\bar{x}$ item score of these items of the group of $25 \%$ of the lowest Total A.S.A.-A scores

H.4. The percentage of explained variance of the first component will be twice the percentage of explained variance of the second component on the unrotated matrix of the factor analysis of the A.S.A.-A scale

Studies

I ELPO, II RAP, III

REHAB, IV HOP, V

DIHOP, VI CABS, VII

COLS; (I till VI),

(I till VII)

(I till VI), VII

COLS, (I till VII)

(I till VI), VII

COLS, (I till VII)
(I till VI), VII

COLS, (I till VII) 
H.5. Item to factor correlations of all 24 items on the first extracted component of the unrotated matrix of the factor analysis of the A.S.A.-A scale will be $\geqslant .35$

H.6. Subjects' Total A.S.A.-A scores will significantly positively $(>.30,<.70)$ correlated to these subjects' Total A.S.A.-B scores given by their significant others

H.7. Patients' Total A.S. A.-A scores will be significantly positively $(>.30,<.70)$ correlated to these patients' Total A.S.A.-B scores given by nurses, who take care of these patients on a regular basis

H.8. Subjects' Total A.S.A.-A scores will be significantly strongly and positively $(\geq .70)$ correlated when measured with a six weeks time-interval in which no major critical life events were experienced by them

H.9. Subjects' Total A.S.A.-A scores will be significantly moderately and positively $(\geq .40)$ correlated when measured with a six months time interval in which no major critical life events were experienced by them
(I till VI), VII

COLS, (I till VII)
I ELPO, II RAP, III REHAB, IV HOP, VI

CABS, (I + II + III

+ IV + VI)

I ELPO, II RAP, till IV)

\section{$\mathbb{I E L P O}$}

\section{CABS}

Construct Validity

Hypotheses

H.10. $\overline{\mathrm{x}}$ Total A.S.A.-A score of Elderly living independently in the community will be significantly higher than $\bar{x}$ Total A.S.A.-A score of Service-Flat residents

H.11. $\overline{\mathrm{x}}$ Total A.S.A.-A score of Service-Flat residents will be significantly higher
Studies

I ELPO

I ELPO 
than Total A.S.A. A score of Personalised Care Facility Residents

H.12. $\overline{\mathrm{x}}$ Total A.S.A.-A score of Personalised I ELPO

Care Facility Residents will be significantly higher than $\overline{\mathbf{x}}$ Total A.S.A.-A score of Nursing Home Residents

H.13. Subjects' Total A.S.A.-A scores will be significantly positively and moderately $\geq .40$ correlated to Total shortened Version (16 items) ESCAS scores.

H.14. Subjects' Total A.S.A.-A scores will be significantly positively and weakly to moderately $(\leq .40)$ correlated to General Assessment of Self-Care Agency scores

H.15. Chronic patients and elderly patients' Total A.S.A.-A scores will not be significantly correlated with these patients' Total A.D.L.-scores

H.16. Patients' Total A.S.A.-A scores will be significantly positively correllated to their Total R.P.R.I.-scores

III REHAB

I ELPO, II RAP, IV HOP, V DIHOP, VII COLS, $(\mathrm{I}+\mathrm{II}+\mathrm{IV}$ $+\mathrm{V}+\mathrm{VII})$

II RAP, V DIHOP

VI CABS

H.17. C.A.B.S.-patients" 6 month post-operative VI CABS $\overline{\mathrm{x}}$ Total A.S.A.-A score will be significantly higher than the same patients preoperative $\tilde{x}$ Total A.S.A.-A score

H.18. C.A.B.S.-patients' 12 month post-operative VI CABS $\overline{\mathrm{x}}$ Total A.S.A.-A score will be significantly higher than the same patients preoperative $\bar{x}$ A.S.A.-A score

H.19. Chronic patients" Total A.S.A.-A scores will be significantly positively correlated to Total Functional and Network N.S.S.Q.-scores

H.20. Subjects' Total A.S.A.-A scores will be significantly but weakly positively or

II RAP

VII COLS 
negatively $(<.30)$ correlated to Total

G.R.A.S. scores.

H.21. Total A.S.A.-A scores of subjects older

$(\mathrm{I}+\mathrm{II}+\mathrm{III}+\mathrm{IV}+$ than 65 years will be significantiy nega$\mathrm{V}+\mathrm{VI})$ tivelly correlated with their age

Measurement Conditions

Hypotheses

Studies

H.22. Subjects' Total A.S.A.-A scores will not

VI CABS

be significantly correlated to these subjects Total scores on the adapted

C. \& M. Social Desirability scalle

H.23. $\overline{\mathrm{x}}$ Total A.S.A.-A score of subjects to VI CABS whom the scale is personally administered will not significantly differ from $\overline{\mathbf{x}}$ Total A.S.A.-A score of comparable subjects to whom the scale is send by mail 


\section{RESULTS}

\section{Introduction}

This chapter describes results from the program of research presented in chapter four. Results are given from meta-analysis of the combined data-set of all studies as well as results from data-analysis of study I: ELPO, study II: RAP, study III: REHAB, study IV: HOP, study V: DIHOP, study VI: CABS, study VII: COLS separately. Results are presented in relation to the hypotheses formulated in the third chapter.

Descriptive statistics on Total A.S.A.-A scores are given first. Results for hypotheses formulated to test reliability are subsequently presented for internal consistency, equivalence and stability. Evidence in terms of construct validity is given for the known groups approach and for testing of convergence and discriminability. This section is followed by results from tests of theoretical relationships and by results from exploration of factor analysis. The chapter ends presenting results for hypotheses formulated to test effects of certain measurement conditions on A.S.A.-A scores.

\subsection{Total A.S.A.-A scores}

Theoretically Total A.S.A.-A scores can range from 24 until 120 . For the entire population of the research program $(n=676)$ the empirical range was from 37 until 117. Only Total A.S.A.-A scores of first measurements were included. In this frequency distribution the mode was 86 , the median 90 and the mean 89.6. The shape of the distribution is unimodel and negatively skewed (see figure 5.1 .).

Skewness of the distribution was -0.43 and kurtosis 1.2 . Only a small percentage of the Total A.S.A.-A scores in the entire population of the research program was lower than 60 . For the subsample of predominantly older subjects with health problems (studies I till VI) the range was the same as in the entire sample, as well as mode (86), median (90) and mean (89.6). Skewness $(-0.58)$ and kurtosis (1.56) were larger, indicating a more negatively skewed and less homogeneous distribution. The shape of the frequency distribution of the 
Figure 5. 1. Frequency Distribution Tota A S.A. A of entire study population

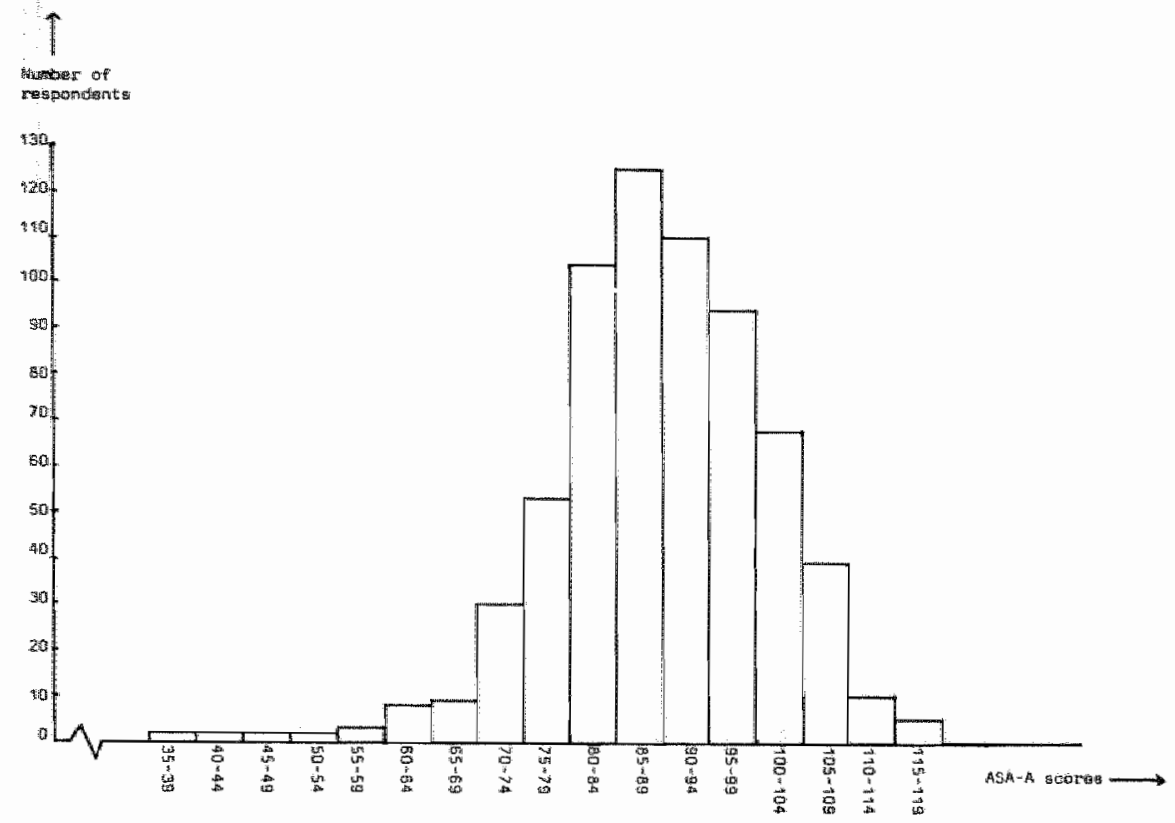

subsample of predominantly young healthy subjects (study VI) was different. Total A.S.A.-A scores ranged from 61 until 115. The mode was 90 , the median 89 , the mean 88.9 . Skewness was -0.03 and kurtosis 0.22 . This is indicative for a less skewed more normal shape of the frequency distribution and greater homogeneity in scoring.

In order to further investigate negative skewness of the frequency distribution of Total A.S.A.-A scores data were analysed on item level. Table 5.1. gives for all 24 items of the A.S.A.-A scale the percentages of subjects scoring (total) agreement with a positively stated item and scoring (total) disagreement with a negatively stated item. Both stand for agreement with operability of Self-Care Agency

Percentages are given for data of the combined studies I-VII, I-VI and all individual studies. The percentages $\geq 85$ are underlined in this table pointing out extremely skewed item score distibutions. If consistent across studies these high percentages indicate low discriminative power of the item and a contribution of the item score to negative skewness of Total A.S.A.-A score distribution. Only item number 4 (maintenance of sanitary conditions) shows percentages in the nineties with a consistent pattern across all studies. 
Table 5.1. A.S.A.A A them scores indicating agrement with operability of Self-Care Agency (in \%)

\begin{tabular}{|c|c|c|c|c|c|c|c|c|c|c|}
\hline $\begin{array}{c}\text { It dian: } \\
\text { \# }\end{array}$ & $\begin{array}{l}\text { Tent } \\
\text { netrore }\end{array}$ & $\begin{array}{l}\mathbb{I}-4 \mathbb{I} \\
4 W \times 8 \%\end{array}$ & $\begin{array}{l}(-k 1 \\
(4)=453)\end{array}$ & $\begin{array}{l}\text { I-ELPO } \\
\text { I }\end{array}$ & $\begin{array}{l}I I-S A P \\
(H=4 A)\end{array}$ & 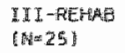 & $\begin{array}{l}\mathrm{TH}-\mathrm{HOP} \\
\text { (N=60) }\end{array}$ & $\begin{array}{l}y-\mathrm{DHHOP} \\
(N=7>)\end{array}$ & $\begin{array}{l}U I-C A E S \\
(N=107)\end{array}$ & 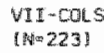 \\
\hline 1 & $*$ & 63 & 84 & 80 & 95 & 79 & 要 & $6 \sqrt{3}$ & sis & 80 \\
\hline 2 & - & 52 & 53 & 60 & 5.2 & 4 & $g 8$ & $59:$ & 34 & 49 \\
\hline 3 & 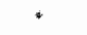 & 77 & 0 & 70 & $B B$ & 33 & 87 & 93 & 78 & $B 9$ \\
\hline 4 & * & 33 & 914 & 8 & 95 & 98 & 100 & 95 & 97 & 90 \\
\hline 9 & ; & 62 & 71 & 64 & 91 & 72 & 30 & 81 & 93 & 43 \\
\hline 6 & $*$ & 6月 & $5 \mathrm{~B}$ & $x_{1}$ & 20 & 65 & 45 & 54 & 5 & 74 \\
\hline 7 & $*$ & $\sqrt{3} 3$ & 54 & 30 & 77 & 64 & $\mathrm{~B} 3$ & 55 & 24 & 50 \\
\hline$\theta$ & * & 82 & 81 & 70 & 84 & 88 & 86 & 89 & $\underline{95}$ & 83 \\
\hline 맘 & * & 71 & 76 & 84 & 75 & 55 & 80 & 71 & 72 & 64 \\
\hline 10 & * & 79 & 71 & 狏施 & 82 & 68 & 58 & 74 & 75 & 89 \\
\hline 14 & * & 59 & Gif & 61 & 82 & $4 \mathrm{~B}$ & 87 & 60 & 33 & 50 \\
\hline 12 & $*$ & 157 & E[व] & 40 & 39 & 85 & 35 & 52 & 77 & 82 \\
\hline 13 & 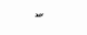 & 80 & 62 & 73 & 36 & 52 & 57 & 64 & 61 & 55 \\
\hline 1.4 & - & 76 & 72 & 73 & 75 & 76 & 72 & 79 & 8.4 & 84 \\
\hline 15 & - & 53 & 47 & 4.64 & 4.5 & 44 & 47 & 57 & 4.2 & 65 \\
\hline 10 & + & 74 & 74 & SB & 暗 & 89 & 78 & 82 & 274 & 00 \\
\hline 17 & + & 162 & 61 & 45 & 64.4 & 52 & 酯的 & 76 & 20 & 63 \\
\hline 18 & * & 77 & 83 & 72 & 95 & 76 & 35 & 93 & Bis & 60 \\
\hline 19 & + & 54 & 565 & EG & 5.4 & 55 & 87 & 58 & 47 & 42 \\
\hline 20 & - & 76 & 70 & 80 & 6.4 & 72 & 72 & 82 & 49 & 90 \\
\hline 24 & + & 82 & 85 & 88 & 79 & 90 & 9 & 84 & 82 & 77 \\
\hline 22 & + & 84 & $\underline{86}$ & 90 & 79 & 72 & 90 & 93 & 4 & 79 \\
\hline 23 & - & 79 & 77 & 87 & 77 & 72 & 72 & 87 & 81 & Bit \\
\hline 24 & - & 70 & 61 & 53 & 32 & 72 & 43 & 75 & 80 & 90 \\
\hline
\end{tabular}

The range and standard deviation of Total A.S.A.-A scores of study I: ELPO (37-117, SD 15.3) were largest of all studies in the research program. Thus Total A.S.A.-A scores in this study with elderly showed a greater amount of variability than those in other studies. Study IV: HOP demonstrated the smallest range (71-104) and SD (7.5) on Total A.S.A.-A scores (see table 5.2). This is indicative for highest degree of homogeneity in scores of acute care hospital patients compared to the other studies.

The lowest mean Total A.S.A.-A score (87.7) was produced in study IV: HOP by patients in an acute care hospital. The highest mean score $(92.6)$ was produced by chronic reumatoid arthritic patients at home. Statistics of study I: ELPO combine Total A.S.A.-A scores of four different sub-samples of elderly. An SD of 15.3 is indicative for a great variety in Total A.S.A.-A scores in this study. The sub-sample of elderly with highest mean Total A.S.A.-A score (100.9) was living independently in the community $(n=40)$. The sub-sample of elderly with lowest mean Total A.S.A.-A score (80.6) staying in a nursing home $(n=40)$. As a consequence the difference between highest and lowest mean 
Table 5.2. Descriptive Statistics Total A.S.A.-A studies 1-VII

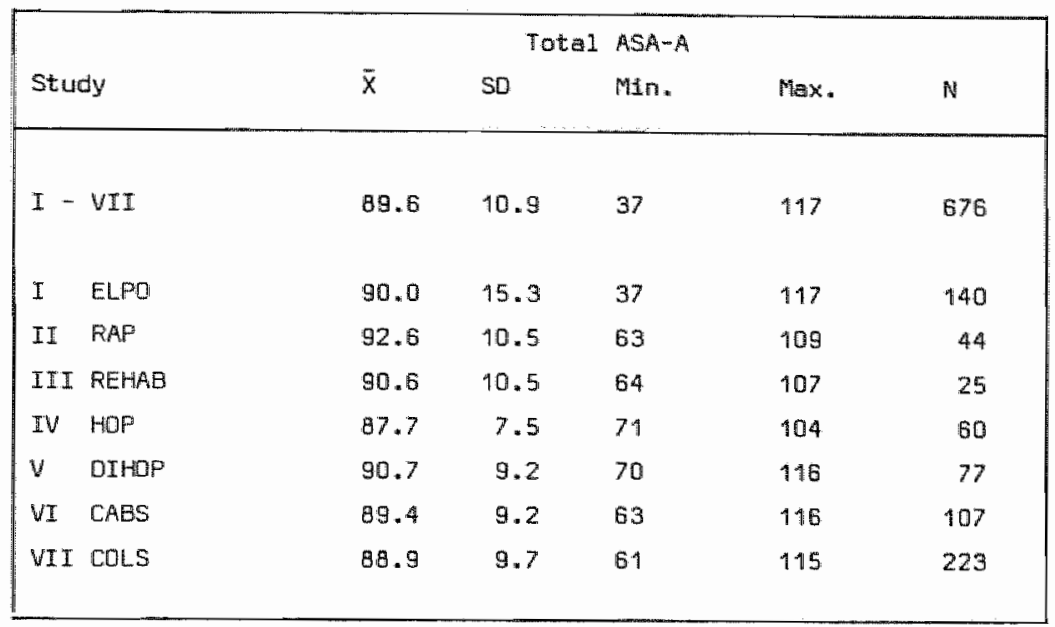

group score in this study was 20.3 . When study I: ELPO is left aside, in this program of research-studies the difference between highest and lowest mean group score on Total A.S.A.-A was 4.9. Mean group scores of nursing home patients (study I: ELPO), of acute care hospital patients (study IV: HOP), of college students (study VII: COLS) and hospitalized Coronary Artery Bypass patients (study VI: CABS) were in a declining sequence lower than the mean score on Total A.S.A.-A of the entire population of the research program. Mean group scores of rehabilitation patients (study III: REHAB), of discharged patients (study V: DIHOP) of chronic reumatoid arthritic patients at home (study II: RAP) and of elderly living independently at home (study I: ELPO) were in an up-climbing sequence higher than the mean Total A.S.A.-A score of the entire study population.

\subsection{Internal consistency}

The first hypothesis formulated to test internal consistency of the A.S.A.-A scale expects Cronbach's alpha's to be $\geq .70$. Calculations were done on the combined data of studies I-VII, studies I-VI and on data of the individual studies. Table 5.3. shows results of these calculations. The alpha of A.S.A.-A measurement on the entire study population was .77. The alpha's for the subsamples of healthy young subjects study VII and of older subjects with health problems (studies I-VI) were similar. The lowest alpha's were calculated in study IV for measurement on acute care hospitall patients and in study VI for measurement with coronary artery bypass surgery patients. Alpha's were calculated for first measurements. In study VI: CABS alpha for measurement at six months post-operative was .75 and at twelve months post-operative .78 . 
Table 5.3. Cranbach's Alpha A.S.A. A Measurements

\begin{tabular}{|c|c|c|}
\hline MEESUREMEAT & $\begin{array}{l}\text { ALPHA } \\
\text { (STANOAFOTZED) }\end{array}$ & $\mathbb{N}$ \\
\hline Study I ELPD & .82 & 135 \\
\hline Study II RAP & .72 & 44 \\
\hline Study MII REHAE & .73 & 25 \\
\hline Study IV HOP & .69 & 60 \\
\hline Study $M \quad$ DIHOP & .78 & 73 \\
\hline Study WI CABS & .68 & 104 \\
\hline Study VII COLS & .77 & 212 \\
\hline Study I-WI & .77 & 4.4 \\
\hline Study I-VII & .76 & $65:$ \\
\hline
\end{tabular}

Cronbach's alpha was highest for A.S.A.-A measurement with elderly (study I: ELPO). Calculations were also done for the subsamples of elderly in this study. Highest alpha (.92) was calculated for measurement with elderly staying in a nursing home. With only two borderline cases results support the first hypothesis clearly.

The second hypothesis expects item to total correlations of all 24 items to be $\geq 30$. Table 5.4. gives the results of calculations of corrected item to total correlations for all 24 items. Calculations were done on data of the entire population of the research program. (Studies I-VII) of the subsample of older subjects with health problems (studies I-VI) and of the subsample of young healthy subjects (study VII). Corrected item to total correlations with a value < .30 are marked in the table. The following items demonstrate low correlations to Total A.S.A.-A scale with a consistent pattern across calculations: Item number 6 (sufficient energy), number 7 (seeking improvement), number 10 (managing to be alone), number 12 (having a circle of friends), number 13 (getting enough sleep), number 24 (no mobility limitations). Eleven items demonstrate consistently moderate to strong item to total correlation: $\mathrm{nr} .1$ (making adjustments), nr.2 (checking measures), nr.3 (making mobility related adjustments), nr.4 (maintenance of sanitary conditions), nr.14 (asking for clarifications), $\mathrm{nr} .15$ (body examination), $\mathrm{nr} .16$ (obtaining medication information), $\mathrm{nr} .17$ (changing old habits), nr.19 (effectiveness evaluation), $\mathrm{nr} .20$ (taking time for self-care), $\mathrm{nr} .21$ (getting health related information). The second hypothesis is consistently supported for $11 \mathrm{items,} \mathrm{partly} \mathrm{supported} \mathrm{for} 7$ items, and consistently not supported for 6 items. 
Table 5.4. Corrected Item to Total Correlations A.S.A.A scale

\begin{tabular}{|c|c|c|c|}
\hline $\begin{array}{l}\text { ASA-A } \\
\text { Item } \#\end{array}$ & $\begin{array}{l}\text { Item to total } \\
\text { correlation } \\
\text { for study I-VI }\end{array}$ & $\begin{array}{l}\text { Item to total } \\
\text { correlation } \\
\text { for stiudy VII }\end{array}$ & $\begin{array}{l}\text { Item to total } \\
\text { correlation } \\
\text { for study I-VII }\end{array}$ \\
\hline 1 & .41 & .33 & .39 \\
\hline 2 & .33 & .38 & .35 \\
\hline 3 & .37 & .35 & .37 \\
\hline 4 & .44 & 30 & .40 \\
\hline 5 & .38 & $.18^{x}$ & .33 \\
\hline 6 & $.16^{x}$ & $.28^{x}$ & $.18^{\times}$ \\
\hline 7 & $.14^{x}$ & $.09^{x}$ & $.13^{x}$ \\
\hline 8 & .37 & $.22^{x}$ & .32 \\
\hline 9 & $.12^{x}$ & .31 & $.18^{x}$ \\
\hline 10 & $.27^{\times}$ & $.29^{x}$ & $.28 \times$ \\
\hline 11 & $.29^{x}$ & .32 & .30 \\
\hline 12 & $.22^{x}$ & $.23^{x}$ & $.20 \times$ \\
\hline 13 & $.11^{x}$ & $.28^{x}$ & $.16^{x}$ \\
\hline 14 & .33 & .38 & .32 \\
\hline 15 & .33 & .32 & .31 \\
\hline 16 & .39 & .30 & .36 \\
\hline 17 & $.13^{x}$ & .32 & $.18^{x}$ \\
\hline 18 & .47 & .38 & .44 \\
\hline 19 & .36 & .47 & .38 \\
\hline 20 & .36 & .34 & 34 \\
\hline 21 & .47 & .31 & .43 \\
\hline 22 & $.20^{\times}$ & .30 & $.23^{x}$ \\
\hline 23 & $.20^{x}$ & .35 & .30 \\
\hline 24 & $.20^{x}$ & $.96^{x}$ & $.15^{x}$ \\
\hline
\end{tabular}

x: Item to tatel correlation $<.30$

The third hypothesis expects all mean item scores of $25 \%$ of the highest Total A.S.A.-A scores to be significantly higher than the mean score of comparable items of $25 \%$ of the lowest Total A.S.A.-A scores. T-tests were calculated on data of the entire study population (studies I-VII), of the older subjects with health problems (studies I-VI) and young healthy subjects (study VII). Table 5.5. shows the results. All $t$-values are significant at $<.01$. With the exception of 
items nr. 5 and 7 in study VII $t$-values are significant at $p<.001$. These results demonstrate, that hypothesis three is strongly supported.

Table 5.5. Difference in X Item Score between high and low Total A.S.A.-A scores

\begin{tabular}{|c|c|c|c|}
\hline $\begin{array}{l}\text { Item \# } \\
A S A-A\end{array}$ & $\begin{array}{l}T \text {-value }(d f) \\
\text { Study } I-V I\end{array}$ & $\begin{array}{l}\text { T-value (df) } \\
\text { Study VII }\end{array}$ & $\begin{array}{l}\text { T-walue (df) } \\
\text { Study I-VII }\end{array}$ \\
\hline 1 & $-9.51 * *(171)$ & $-5.68 * *(113)$ & $-914.59 * * *(278)$ \\
\hline 2 & $-0.87 * *(240)$ & $-8.23 * *(115)$ & $-11.68 * * *(354)$ \\
\hline 3 & $-7.68^{* * *}(203)$ & $-5.89 *(116)$ & $-9.60 * * *(326)$ \\
\hline 4 & $-0.19 * *(136)$ & $-5.25 *(106)$ & $-9.66 * *(249)$ \\
\hline 5 & $-9.65 * *(215)$ & $-2.89 *(124)$ & $-9.65^{* * *}(358)$ \\
\hline 6 & $-5.10 * * *(240)$ & $-5.60 *(111)$ & $-7.25^{*} *(360)$ \\
\hline 7 & $-5.17 * *(240)$ & $-2.73^{* *}(125)$ & $-5.93 *(360)$ \\
\hline$\theta$ & $-7.00 * * *(190)$ & $-5.73 * * *(95)$ & $-9.71 *(294)$ \\
\hline 9 & $-4.96 * *(239)$ & $-6.00 * * *(106)$ & $-7.64 * *(334)$ \\
\hline 10 & $-7.01 * * *(221)$ & $-6.20 * *(100)$ & $-9.21 * * *(334)$ \\
\hline 11 & $-6.15 * * *(223)$ & $-6.88^{* * *}[125]$ & $-10.72 * *(346)$ \\
\hline 12 & $-6.11 * * *(239)$ & $-3.91 * *(106)$ & $-6.53^{* * *}(358)$ \\
\hline 13 & $-4.25^{* * *}(240)$ & $-4.95 * * *(125)$ & $-6.41 * *(360)$ \\
\hline 14 & $-9.11 *(213)$ & $-6.25 * * *(107)$ & $-10.70 * *(326)$ \\
\hline 15 & $-8.85^{* * *}[240]$ & $-6.71 * * *(119)$ & $-10.51 * * *(360)$ \\
\hline 16 & $-10.11 * *[211]$ & $-5.80 * * *(101)$ & $-11.04 * *(316)]$ \\
\hline 17 & $-4.09 * *(240)$ & $-6.48 *(112)$ & $-6.04 * *(360)$ \\
\hline 18 & $-11.25 * *(152)$ & $\sim 6.57 * * *(119)$ & $-13.02 *(284)$ \\
\hline 19 & $-9.20 * * *(239)$ & $-10.73 * * *(125)$ & $-12.73 * *(359)$ \\
\hline 20 & $-9.55^{* * *}(213)$ & $-5.69 * * *(99)$ & $-10-86 * *(322)$ \\
\hline 24 & $-10.89 * *(159)$ & $-5.97 * *(120)$ & $-12.44 * * *(283)$ \\
\hline 22 & $-6.00 * *(204)$ & $=4.3 \theta^{* * *}(115)$ & $-7.73^{* * *}(322)$ \\
\hline 23 & $-8.00^{* * *}[206]$ & $-6.18^{* * *}(118)$ & $-9.79 * *(329)$ \\
\hline 24 & $-6.30^{* * *}(240)$ & $-3.14 * *(116)$ & $-6.41 * * *(357)$ \\
\hline
\end{tabular}

\footnotetext{
* p $<.05$

$* p<0.01$

$*$ * p $<0.001$
} 
Hypothesis four states that the percentage of explained variance of the first component will be twice that of the second component on the unrotated matrix of the factor analysis of A.S.A.-A scores. Table 5.6. gives the results of factor analysis done on data of all first A.S.A.-A measurements of the entire research program population and of the subsample of older subjects with health problems (studies I-VI) and the subsample of young healthy subjects (study VII). Percentages are given for factors with eigenvalue $\geq 1.00$.

Table 5.6. Factor Anulysis (unrotated) A.S.A.-A scale

\begin{tabular}{|c|c|c|c|c|c|c|}
\hline \multirow[t]{2}{*}{ Factor } & \multicolumn{2}{|c|}{ Study I-VI } & \multicolumn{2}{|c|}{ Study VII } & \multicolumn{2}{|c|}{ Study I-VII } \\
\hline & Eigenwalue & \& vartance & Elgervalue & \% variance & Elgenvelue & wartanci \\
\hline 1 & 4.16 & 97.3 & 3.91 & 16.3 & 3.96 & 16.5 \\
\hline 2 & 2.35 & 9.8 & 2.22 & 9.2 & 2.16 & 9.0 \\
\hline 3 & 1.79 & 7.5 & 4.62 & 0.8 & 4.65 & 15.9 \\
\hline 4 & 1.41 & 5.9 & 1.48 & 6.2 & 1.53 & 6.4 \\
\hline 5 & 1.20 & 5.0 & 1.34 & 5.6 & 1.28 & 5.4 \\
\hline 6 & 1.14 & 4.8 & 1.23 & 5.1 & 1.09 & 4.6 \\
\hline 7 & 9.05 & 4.4 & 1.08 & 4.5 & 1.05 & 4.8 \\
\hline$\theta$ & & & 1.05 & 4.4 & & \\
\hline Totai & 13.10 & 54.6 & 13.93 & 50.0 & 12.74 & 53.1 \\
\hline
\end{tabular}

The first factor in the data-analysis of studies I-VII explains $16.5 \%$, in dataanalysis of studies I-VI $17.3 \%$ and in that of study VII $16.3 \%$. The percentages of explained variance of the second factor are $9 \%, 9.8 \%$ and $9.2 \%$ respectively. The ratio explained variance first factor: second factor is respectively 1.83,1.77 and 1.77. All three approach 2.00. Table 5.6. further shows quite simillar only slightly decreasing percentages of explained variance for the rest of the extracted factors. Though strictly speaking hypothesis four is not supported, results do indicate the presence of a clear first factor. Results may also indicate the presence of a possible meaningfull second factor.

Hypothesis five expects all item to factor correlations (loadings) to be $\geq .35$ on the first extracted component of the unrotated factor analysis of the A.S.A.-A scale. Table 5.7. shows the loadings on the first factor. Results are given for the A.S.A.-A scale factor-analysed on data from the combined studies I-VII, I-VI and from study VII. Items with factor correlations of $<.35$ are marked. 
Table 5.7. Ttem loadings on First Factor of A.S.A. A. Factor Analysis (unrotated)

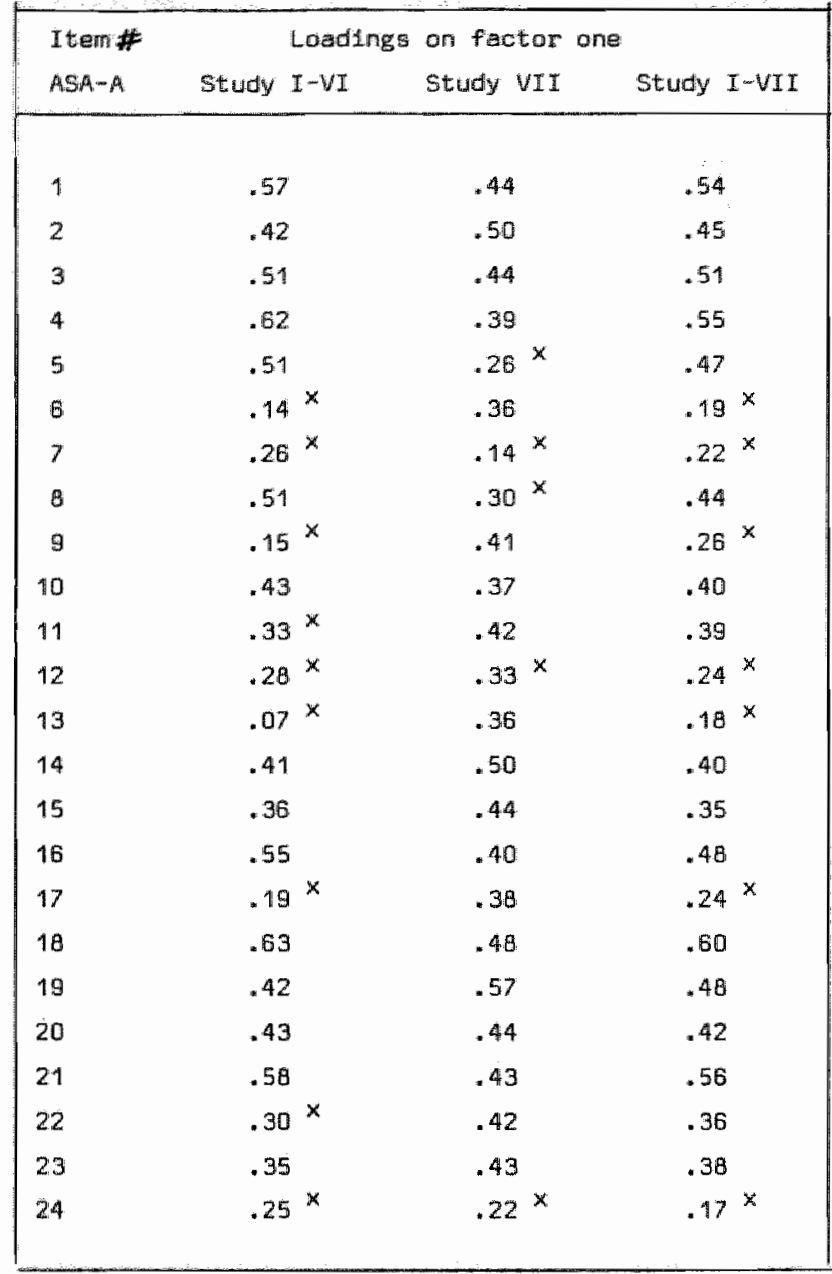

$x$ : Itam to factor correlation $<.35$

Table 5.7 shows that all item loadings on the first extracted factor on the unrotated matrix are positive. Item nr.18 (taking safety measures) has the highest loading (.60) on the first factor in the analysis of data of the entire research program population as well as in the data analysis of the subsample of older subjects with health problems (.63). In the analysis of the subsample of young healthy subjects it is item nr. 19 (evaluating effectiveness). Items having 
lowest loadings on the first factor were respectivelly. nr.24 no mobillity limitations (.17) (Study I-VII), nr. 13 getting enough sleep (.07) (Study I-VI) and nr.7 seeking improvement (.14) (Study VII). Three items demonstrate loadings on a first factor of $<.35$ consistently across the factor analyses: $\mathrm{nr} .7$ (getting enough sleep), nr.12 (having a circle of friends) and nr.24 (no mobility limitation). Thirteen items consistently demonstrate correlations of $\geq 35$ : item nr. 1 (making adjustments), nr.2 (checking measures), nr.3 (making mobility related adjustments), nr.4 (maintenance of sanitary conditions), nr.10 (managing to be alone), $\mathrm{nr} .14$ (asking for clarification), nr.15 (body examination), nr.16 (obtaining medication information), $\mathrm{nr} .18$ (taking safety measures), $\mathrm{nr} .19$ (effectiveness evaluation), nr.20 (taking time for self-care), $\mathrm{nr} .21$ (getting health related information), $\mathrm{nr} .23$ (having time for self). These results indicate that hypothesis five is consistently supported for 13 items, partly supported for 8 items and consistently not supported for 3 items.

\subsection{Equivalence}

Hypothesis six predicts a significantly positive correlation $r>.30$ but $<.70$ between subjects Total A.S.A.-A scores and their Total A.S.A.-B scores given by significant others. Table 5.8. gives Pearson's correlation coefficients calculated for all subjects in the research program for whom a Total A.S.A.-A score of a significant other was available and in relevant studies separately. In study I these subjects were elderly living independently in the community, in study II reumatoid arthritic patients at home, in study III patients staying in a physical

Table 5.8 Correlations A.S.A.-A Subjects-A.S.A.-B Significant Others

\begin{tabular}{|llll|}
\hline Study \# & & $\begin{array}{l}\text { Pearson's r } \\
\text { ASA-A - ASA -B } \\
\text { sign. others }\end{array}$ & N \\
& & & \\
Study I & ELPO & $.54 * * *$ & 37 \\
Study II RRAP & $.70^{* * *}$ & 39 \\
Study III REHAB & .32 & 23 \\
Study IV HOP & $.72 * * *$ & 60 \\
Study VI CABS & $.43^{* * *}$ & 60 \\
& & & 219 \\
Tata1 & & $.38 * * *$ & \\
\hline
\end{tabular}

***) $p<.001$

$n s=$ not significant 
rehabitation center, in study IV patients staying in an acute care hospital and in study VI patients admitted for coronary bypass surgery. In case of longitudinal studies first measurements of A.S.A.-A and A.S.A.-B were used.

Table 5.8 shows that all correlation coefficients are positive and higher than .30 . With the exception of Study IV HOP they were not higher than .70. The lowest coefficient was calculated for rehabilitation patients the highest for patients staying in an acute care hospital. All correlations are significant at $p<.001$ with an exception of the one calculated for the rehabilitation patients. Hypothesis six is supported with one exception.

Hypothesis seven also predicts a significantly positive correlation $r>.30$ but $<.70$. In this case it concerns Total A.S.A.-A scores of patients and their Total A.S.A.-B scores given by nurses who are taking care of these patients on a regular basis "In table 5.9 Pearson's correlation coefficients are given for these pairs. Again a coefficient was calculated for all patients in the research program for whom Total A.S.A.-B scores of nurses were available and for nursing home patients (study I: ELPO), for chronic reumatoid arthritic patients staying at home (study II: RAP), for patients staying in a rehabilitation center (study III: REHAB) and for patients staying in an acute care hospital (study IV: HOP).

Table 5.9 Correlations A.S.A.-A Parients-A.S.A.-B-Nurses

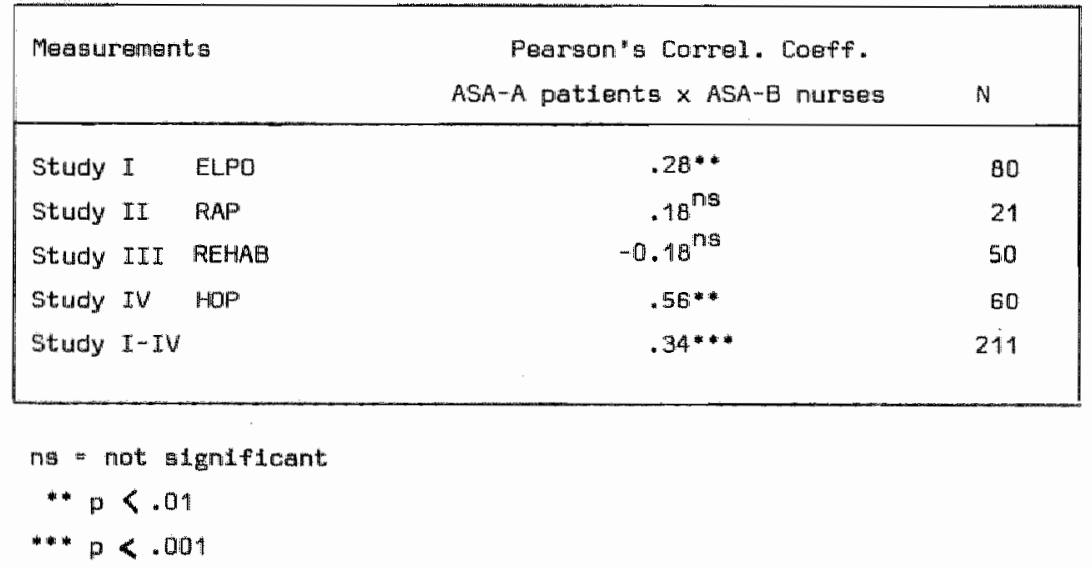

Pearson's correlation calculated for all pairs of patients and nurses combined in the research program is significantly positive and moderate $(r .34)$. For the separate studies three correlation coefficients are positive with one negative exception calculated for the patients in the rehabilitation center $(r=-0.18)$. The lowest positive coefficient $(r=.18)$ was calculated for the reumatoid arthritic 
patients at home, the highest $(r=.56)$ for patients staying in an acute care hospital. Coefficients of reumatoid arthritic and rehabilitation patients were not significant at a $p<.05$ level. In both study I: ELPO and study III: REHAP each patient was rated with A.S.A.-A by two independent nurse raters. A.S.A.-B scores of these two nurses correlated positively strongly in study $\mathbb{I}$ : ELPO $(r=.69)$ and in study III: $\operatorname{REHAB}(r=.71)$ and for all pairs $(r=.78)$. These correlations were significant at a $p<.001$ levell. In order to further investigate equivalence nurses" A.S.A.-B scores were correlated with significant others' A.S.A.-B scores. This was done for all subjects in the research program for whom such a triad of scores was available. A total number of 124 triads could be used. A.S.A.-B scores of nurses correlated moderately positive with A.S.A.-B scores of significant others $r=.31$. Pearson's $r$ was significant at a $p<.001$ level. Results indicate that hypothesis seven is supported for all pairs of patients and nurses taken together in the program of research. This support is not consistent across all studies. In only two of the four studies the hypothesis was supported. This result will be discussed in more detail in chapter six.

\subsection{Stability}

Hypothesis 8 predicts a significantly strong and positive correlation between Total A.S.A.-A scores of elderly living independently in the community when measured with a six weeks time-intervall. Elderly having experienced major critical life events during this period were not included. Table 5.10 shows the calculated Pearson's correlation coefficient.

Table 5.10 Correlation A.S.A.-A for Six Weeks Time Interval

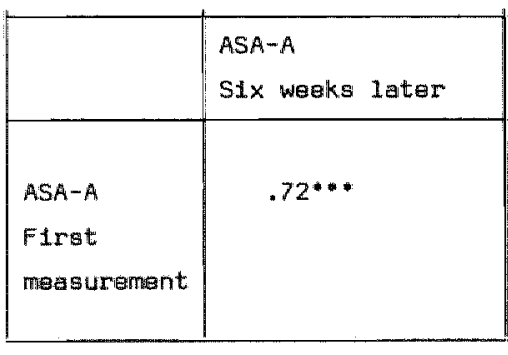

$$
\begin{aligned}
& * * p<.001 \\
& N=36
\end{aligned}
$$

A positive and strong correlation was calculated for 36 elderly in study I: ELPO. Correlation was significant at $p<.001$ level. This result supports hypothesis 8. 
The ninth hypothesis predicts a significantly moderate and positive correlation $r \geq .40$ between Total A.S.A.-A scores of coronary artery bypass patients measured at 6 months and 12 months after their operation. Data were used from study VI: CABS. Table 5.11 shows the results. Pearson's correlation coefficients were calculated for patients together, for patients with low 6 months Total A.S.A.-A scores ( $<88)$ with medium Total A.S.A.-A scores (88-95) and high scores ( $>95$ ). For all patients together correlation was positive and moderate to strong and significant at a $p<.001$ level. This correlation coefficient is lower than the one calculated for a six weeks time-interval in study I: ELPO. The subsamples of patients with low, medium and high scores also show positive but more moderate correlations. Correlation coefficient of the subgroup of patients with medium Total A.S.A.-A scores only reached a significance level of $p<.05$. In order to further investigate stability across this six months post-operative period difference in mean Total A.S.A.-A scores for the six and twelve months post-operative period was calculated using paired t-test practicing two tailed test of significance. For the scores of all $96 \mathrm{CABS}$ patients $t$-test was not significant $T(95)=-0.37$. For patients with low 6 months Total A.S.A.-A scores $(n=32) t$-value $T(31)=-3.03$ indicated a significant $(p<$ $.01)$ increase in mean Total A.S.A.-A score at twelve months after operation.

Table 5.11. Correlation A.S.A.-A for Six Months Time Interval

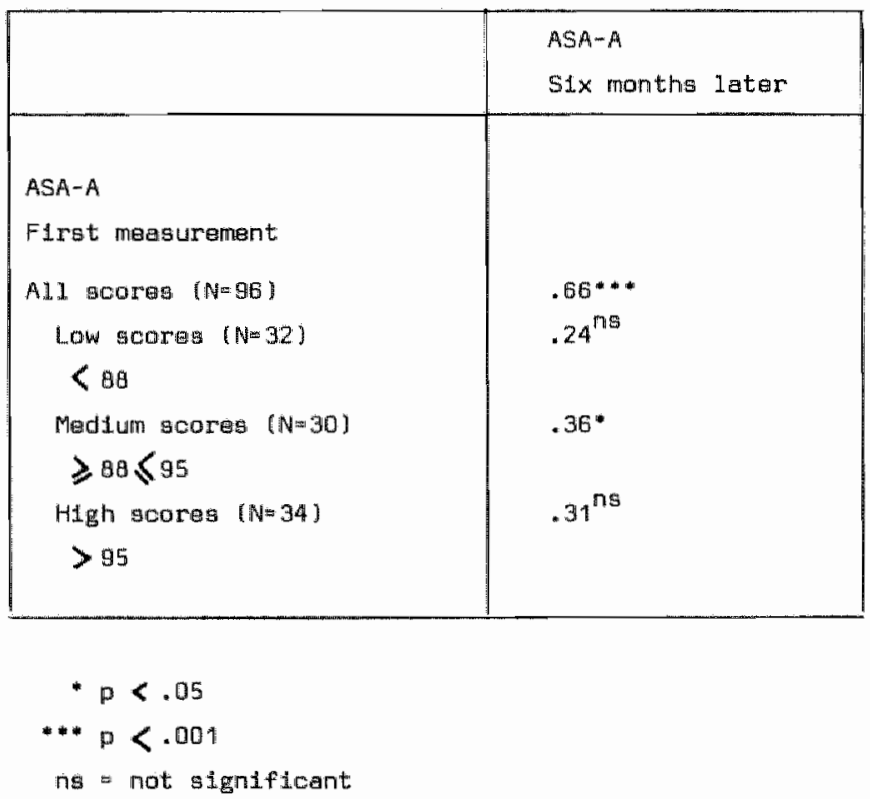


$T$-values for patients with medium scores $T(29)=1.76$ and low scores $T(33)=$ 0.58 were not significant both indicative for no change in mean Total A.S.A.-A scores. These results demonstrate that hypothesis nine is supported for the entire sample of CABS patients and the subsample of CABS patients with medium Total A.S.A.-A scores.

\subsection{Construct Validity}

\section{Known Groups Technique}

Hypothesis ten predicts a higher mean Total A.S.A.-A score for elderly living independently in the community than for service flat residents. Table 5.12 shows the results calculated on data of study I: ELPO.

Table 5.12 Difference $\bar{x}$ Total A.S.A* A scores of Elderly in Community and Service Flaf

\begin{tabular}{|c|c|c|c|}
\hline & \multicolumn{3}{|c|}{ Total ASA - A scores } \\
\hline & $\bar{x}$ & so & t-value $(d f)$ \\
\hline Elderly in community & 100.9 & 9.6 & \\
\hline & & & $3.38(61) p=.001$ \\
\hline Service flat residents & 92.9 & 10.1 & \\
\hline
\end{tabular}

Mean Total A.S.A.-A score of elderly in the community was 100.9 and of service flat residents 92.9 . The difference is significant at $p=.001$ level (one-tailed). This result supports hypothesis ten clearly.

The eleventh hypothesis predicts, that mean Total A.S.A.-A score of service flat residents on its term is higher than the one of personalized care facility residents. Again data were used of study I: ELPO. Results are shown in table 5.13 .

Table 5.13 Difference $x$ Total A.S.A.-A scores Residents Service Flat Personalized Care Facility

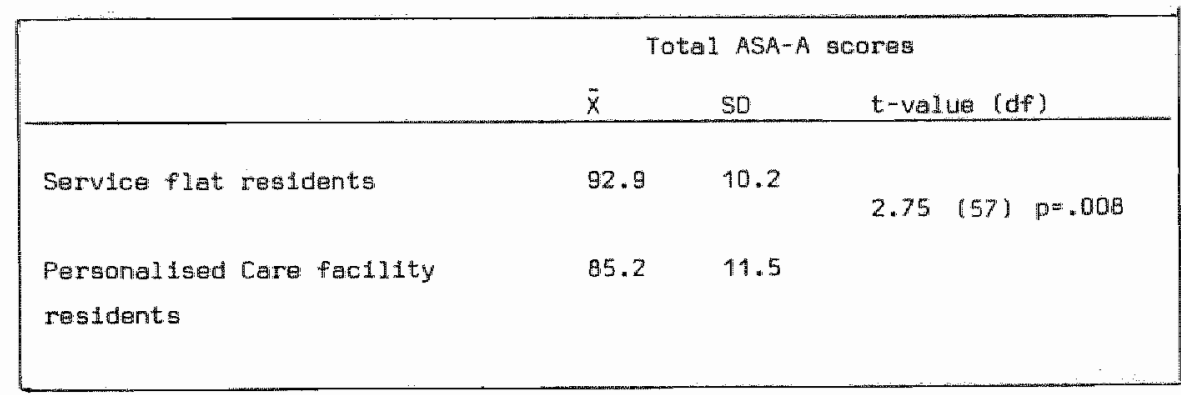


Residents of the personalized care facility had a mean Total A.S.A.-A score of 85.2. When compaired with the mean score of the service flat residents the difference is significant at $p=.008$ level (one-tailed). Hypothesis eleven is supported by this result.

Hypothesis twelve predicts a higher mean Total A.S.A.-A score for residents of the personalized care facility than for nursing home residents. Again data were used of study I: ELPO and a t-test for independent samples was calculated (one-tailed). Table 5.14 shows the result.

Table 5.14 Difference I Total A.S.A.-A scores Residents Personalized Care Facility and Nursing Home

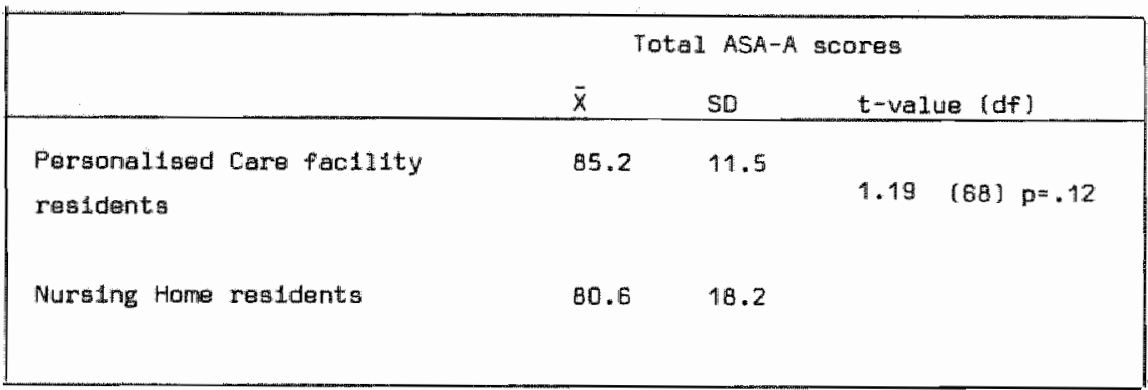

Nursing home residents have a lower mean Total A.S.A.-A score (80.6) than residents of the personalized care facility. Standard deviation of their scores SD $=18.2$ is much higher than SD of scores of personalized care facility residents $(\mathrm{SD}=11.5)$. The difference in mean scores is not significant at $p<.05$ level. Although mean score of personalized care facility residents was higher than the one of nursing home residents, hypothesis twelve is not supported.

In order to further investigate differences between groups mean score of nursing home residents was also compared with mean score of service flat residents and found to be significant $T(68)=3.30, p=.001$. Difference in mean score of nursing home residents and elderly in the community was also significant $T(78)=6.23, p<.001$ as was the difference in mean scores between residents of a personalized care facility and elderly living independently in the community $T(56)=6.10, p<001$. In terms of important background variables the four groups of elderly did not significantly differ for $\operatorname{sex} X^{2}(3)=5.36 p=$ N.S., age $F(3)=1.65, p=$ N.S. and socio-economic status i.e. last occupation $\mathrm{F}$ (3) $=2.57, p=$ N.S. The mean Total A.S.A.-A score of elderly women 96.6 , SD 2.3 was significantly higher $T(138)=2.61, p=.01$ than mean Total A.S.A.-A score of male elderly 88.4, SD 1.5 . Age was also significantly negatively correlated to Total A.S.A.-A $r=-0.22, p=.008$. Last occupation was not significantly correlated with A.S.A.-A $r=-0.02, p=$ N.S. Interaction effect of age and sex on difference between mean group scores was tested by ANOVA 
with sex and age as co-variates. Corrected mean Total A.S.A.-A scores were 99.8 for the elderly living independently in the community, 93.3 for service flat residents, 86.2 for residents of a personalized care facility and 81.1 for nursing home residents. Difference between these corrected mean scores was significant $F(3)=15.97, p=.000$.

\section{Demonstration of Convergence and Discriminability}

Hypothesis thirteen predicts that Total A.S.A.-A scores of physical rehabilitation patients will significantly strongly and positively correlate with their scores on the shortened version of the ESCA-scale. Data were used of study III: REHAB. Table 5.15 shows the result.

Table 5.15 Correlation A.S.A.-A-ESCA

\begin{tabular}{|c|c|}
\hline & 16 items vers. ESCA \\
\hline ASA-A & $.48 * *$ \\
\hline
\end{tabular}

$\mathrm{N}=25$

$*-1 * 0<001$

Pearson's correlation coefficient for Total A.S.A.-A scores and Total scores of the short ESCA version was .48. Correlation was significant at $p<.01$ level. This result supports hypothesis thirteen.

The fourteenth hypothesis predicts a significant weak to moderate $(r<.40)$ correlation between subjects Total A.S.A.-A scores and their scores on Gener. al Assessment of Self-Care capability. Pearson's correlation coefficients were calculated for all subjects in the research program for whom both Total A.S.A.-A and General. Assessment of Self-Care capability scores were available $(n=541)$. Calculations were also done on data of relevant individual studies i.e. study I for elderly, study II for reumatoid arthritic patients, study IV for acute care hospital patients, study $\mathrm{V}$ for discharged patients and study VII for college students. Results are shown in table 5.16.

Correlation coefficient for all subjects in the research program was weakly positive $r=.22$. Significance level was at $p<.001$. Highest correlation calculated on data of the subsamples was .37 and lowest .06 . All correlation coefficients 
Table $\$ .16$ Correlations Total A.S.A. A General Assessment Self-Care Capability

\begin{tabular}{|c|c|c|c|}
\hline Study & m & Peargan's $r$ & ASA- $\times$ Eeneral Assessment \\
\hline I. ELPO & 140 & & $.37 * *$ \\
\hline II RAP & 44 & & $.06^{n s}$ \\
\hline IV MDP & 60 & & $.29 * *$ \\
\hline W DIHOP & 76 & & $.19^{*}$ \\
\hline VII COLS & 221 & & $.28 * * *$ \\
\hline Tata1 & 54.1 & & $.22 * * *$ \\
\hline
\end{tabular}

\footnotetext{
- $<<05$

**p<.01

*** $\mathbb{P}<.001$

$n g=$ not gigniflcant
}

were positive and significant at $p<.05$ level with the exception of the one calculated for chronic reumatoid arthritic patients. Hypothesis fourteen is supported by results for the entire sample of subjects of the research program and by results of all subsamples but one.

Hypothesis fifteen states that Total A.S.A.-A scores of chronic and elderly patients will not significantly be correlated with these patients' Total A.D.L.scores. This hypothesis was tested with chronic reumatoid arthritic patients in study II. Patients' A.D.L. was scored by community nurses and significant others independently. Table 5.17 shows the result.

Table 5.17 Correlation Total A.S.A.A-Total A.D.L. R.A. Patients

\begin{tabular}{|c|c|c|}
\hline & $\begin{array}{l}\text { Sign, ather } \\
\text { Als } 39\end{array}$ & $\begin{array}{l}\text { Comm. Nurse } \\
N=21\end{array}$ \\
\hline $\begin{array}{l}A S A-A \\
\text { pationt }\end{array}$ & $-0.20^{\mathrm{ns}}$ & $-0.05^{n g}$ \\
\hline
\end{tabular}

ns not significant 
Pearson's correlation coefficient for patients' Total A.S.A.-A and Total A.D.L. score given by significant others was $r=-0.20$ and $r=-0.06$ for Total A.S.A.-A and Total A.D.L. scored by nurses. Both correlations were not significant at $p<.05$ level. A.D.L. scores given by significant others and nurses correlated significantly positive and strong $r=.95 p=.000 \mathrm{n}=18$. When Total A.S.A.-A scale was correlated with individual items of A.D.L. score given by significant others two out of six items showed significant negative correlations. These items were respectively Toiletting $r=-0.32, p<.05$ and Feeding $r=$ $-0.27, p<.05$. Thus the lower operability of Self-Care Agency of Reumatoid Arthritic patients was the more dependent they were on assistance with toilletting and feeding. Comparison of Total A.S.A.-A scores with individual items of A.D.L. scores given by community nurses did not show any significant correlations. Hypothesis fifteen was also tested with elderly patients discharged from an acute care hospital. These patients both completed the A.S.A.-A scale and a self assessment scale of A.D.L. Pearson's correlation coefficient was calculated for Total A.S.A.-A and Total A.D.L. Table 5.18 shows the result.

Table 5.18 Correlation Total A.S.A.-A and Total A.D.L. Discharged Patients

\begin{tabular}{|l|l|}
\hline & ADL. \\
\hline ASA-A & $-0.06^{\mathrm{ns}}$ \\
\hline
\end{tabular}

$N=76$

ns $\approx$ not signiflcant

Correlation is nearly zero and not significant. When Total A.S.A.-A score was correlated with individual A.D.L. items again no significant findings were generated. Of all 77 discharged patients 27 received after care and 50 did not. In order to further demonstrate discriminability of A.S.A.-A and A.D.L. scales mean A.S.A.-A and A.D.L. scores were calculated for the two groups of patients i.e. one group receiving and one group not receiving after care. Mean Total A.S.A.-A score for after care patients was $92.3 \mathrm{SD} 9.3$ and for patients without after care 91.0, SD 9.2. Difference in mean scores was not significant $T$ $(50)=-0.33 p=$ N.S. Mean Total A.D.L. score for after care patients was 20.0 , SD 1.5 and for patients without after care 20.7, SD 0.6. Difference between mean A.D.L. scores was significant $T(75)=-2.90, p=.005$. Both groups also differed significantly in terms of their age $T(52)=3.0, p=.004$. Mean age of after care patients was 69.9 years SD 6.9 and of patients without after care 64.9 , SD 6.7. Thus age was also correlated with Total A.D.L. and Total A.S.A.-A. Pearson's correlation coefficients were respectively $r=-0.26, p=.02$ and $r=$ 
$.04, \mathrm{p}=$ N.S. These further analyses give additional evidence for the hypothesis that Self-Care Agency and Activities of Daily living are different concepts, which can be empirically discriminated. Results support hypothesis fifteen.

\section{Examination of Relationships}

According to hypothesis sixteen Self-Care Agency is positively related to Self-Care i.e. measured by Total A.S.A.-A and Total R.P.R.I. scores. This means the more operable Self-Care Agency the more adequate Self-Care. Pearson's correlations were calculated on data from patients experiencing a coronary artery bypass surgery (Study VI: CABS). Results are shown in Table 5.19.

Table 5.19 Correlations Total A.S.A.-A - Total R.P.R.I.scores

\begin{tabular}{|c|c|c|}
\hline & $\begin{array}{r}\text { Persons correlati } \\
\text { ASA-A } \times \text { RPRI }\end{array}$ & eff. \\
\hline \multicolumn{3}{|l|}{ Meas urrement } \\
\hline Pre-operative & $.20^{*}$ & 104 \\
\hline Six-months post-op. & $.19 *$ & 98 \\
\hline TweI ve-mont hs post-op. & $.24 * *$ & 98 \\
\hline
\end{tabular}

- p<.05

* p $<.01$

All correlation coefficients are positive, ranging from $r=.19$ to $r=.24$ and significant at $p<.05$ level. Hypothesis sixteen is supported consistently across all three measurements.

Hypothesis 17 predicts a significant higher six months post-operative than pre-operative mean Total A.S.A.-A score for patients having experienced coronary artery bypass surgery. This hypothesis tests the theoretical relationship health - Self-Care Agency i.e. the effect of positive change in health on Self-Care Agency. T-test for dependent samples (one tailed) was calculated on data of study VI: CABS. Table 5.20 shows the result.

Mean pre-operative Total A.S.A.-A score was 89.4 and mean 6 months postoperative Total A.S.A.-A score was 91.6. Difference is significant at $p<.01$ level. This result supports hypothesis seventeen. 
Table 5.20 Difference in mean Total A.S.A.A pre- and 6 months post-C.A.B.S.

\begin{tabular}{|c|c|c|c|}
\hline Maasurements & Total & $A S A-A$ & t-value $(\mathrm{df})$ \\
\hline CABS patients & $\overline{\mathrm{x}}$ & so & : $\quad$ : : \\
\hline \multicolumn{4}{|l|}{ pre-operative } \\
\hline \multirow[t]{2}{*}{$N=107$} & 89.4 & 9.2 & \\
\hline & & & $-2.74 *(95)$ \\
\hline $\begin{array}{l}6 \text { months post - } \\
N=98\end{array}$ & 99.6 & 8.9 & \\
\hline
\end{tabular}

$* * p<.01$

Hypothesis 18 predicts also a significant higher 12 months post-operative than pre-operative mean Total A.S.A.-A score for coronary bypass surgery patients. Again a t-test for dependent samples was calculated on data of study VI. The result is shown in table 5.21.

Table 5.21 Difference in mean Total A.S.A.-A pre- and 12 months posi-C.A.B.S

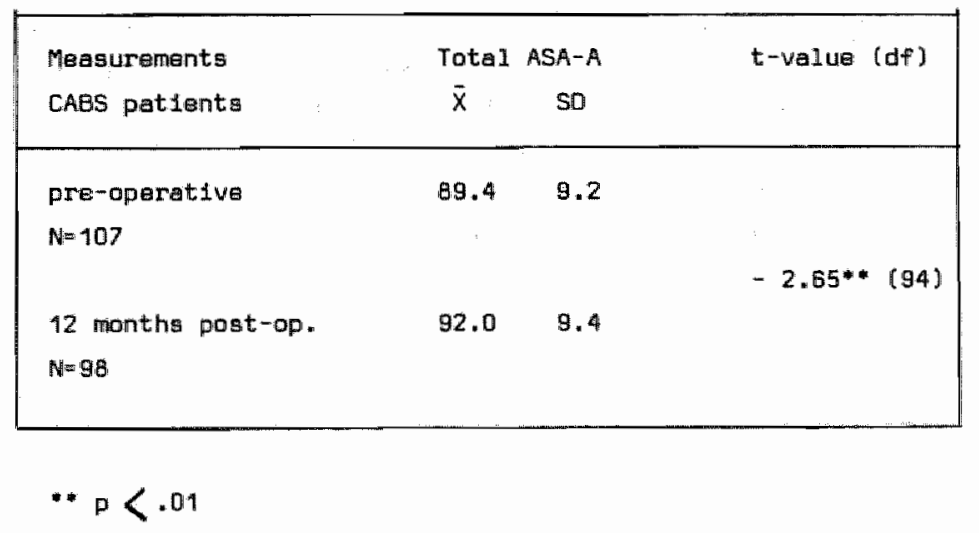

Mean 12 months post-operative Total A.S.A.-A score was 92.0. Difference with pre-operative mean score is again significant at $p<.01$ level. Hypothesis 18 is supported. These last two supported hypotheses indicate evidence for the theoretical positive relationship between health state and Self-Care Agency. 
According to hypothesis nineteen chronic reumatoid arthritic patients' Total A.S.A.-A scores will be significantly positively correlated to their Total Functional and Total Network N.S.S.Q.-scores. Pearson's correlation coefficients were calculated on data of study II. Table 5.22 gives the results.

Table 5.22 Correlation Total A.S.A.-A and N.S.S.Q.-Jubscales

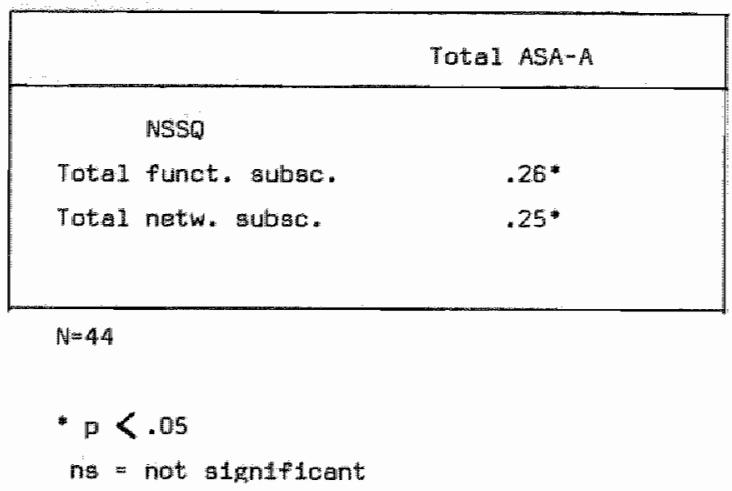

Both Total Functional and Total Network N.S.S.Q. subscales were positively correlated with Total A.S.A.-A scores. Correlations were significant at $p<.05$ level. When correlations of Total A.S.A.-A and individual N.S.S.Q. items are analysed the extend of the social network is significantly positively correlated to operability of Self-Care Agency $r=.30, p=.04$ as to length of time of contacts $r$ $=.27, p=.04$. Feeling respected $(r=.29, p=.03)$ and feeling supported $(r=$ $.30, p=.02)$ are also significantly correlated to Total A.S.A.-A scores. The above results are supportive of hypothesis nineteen. They mean empirical evidence for the proposed positive relationship between perceived social support and operability of Self-Care Agency.

Hypothesis twenty predicts a weak $(r< \pm .30)$ positive or negative correlation between college students Total A.S.A.-A scores and their total G.R.A.S.scores. Data were used from study VII: COLS. Mean G.R.A.S.-score for male college students $(\mathrm{n}=82)$ was $-1.90, \mathrm{SD}=1.91$ and significantly lower $(T(221)=$ $-10.05, p<.001$ ) than mean G.R.A.S. -score of female students $+1.15, \mathrm{SD}=$ 2.33, $\mathrm{n}=141$. School of Business male students had lowest mean G.R.A.S.score $(-2.12, \mathrm{SD}=1.80)$. As such this was reflective of the most masculine stereotyped self-image. This mean score was again significantly lower $(T(84)=$ $-7.91, p<.001)$ than their female collegues' mean score $(+1.47$, SD 2.57). Mean Total G.R.A.S. value for male School of Social Work students was -1.59, $\mathrm{SD}=2.04$ and again significantly different $(T(126)=-6.05, p<.001)$ from the 
mean score of female School of Social Work students $+0.98, S D=2.19$. Based on these Total G.R.A.S.-scores $23 \%$ of all college students were classified as having a stereotype masculine image and a same percentage as having a stereotype feminine self-image. Fourty-six percent were classified as androgyne and $8 \%$ as unclear. Mean Total A.S.A.-A score for all college students was 88.9, $\mathrm{SD}=9.7$. Female college students mean A.S.A.-A score was higher $(89.8, \mathrm{SD}=9.1)$ than male college students' mean $\mathrm{A} . \mathrm{S} . \mathrm{A}$. -A score $(87.8, \mathrm{SD}=$ 10.6). This difference was not significant $T(221)=-1.53, p=N$.S. This finding was consistent for School of Business students: $\bar{x}$ females' Total A.S.A.-A was $89.1, \mathrm{SD}=8.7$ and malles' $\mathrm{x}$ score was $88.0, \mathrm{SD}=10.8, T(93)=-0.52, P=\mathrm{N} . \mathrm{S}$. and also for School of Social Work Students: $\bar{x}$ Total A.S.A.-A score of females $90.2, \mathrm{SD}=9.4, \overline{\mathrm{x}}$ score of males $87.4, \mathrm{SD}=10.5, T(126)=-1.46, P=$ N.S. Table 5.23 shows Pearson's correlation coefficient between Total A.S.A.-A and Total G.R.A.S. calculated for all college students.

Table 5.23 Correlation Total A.S.A.-A - Total G.R.A.S.

\begin{tabular}{|c|c|}
\hline & Total ASA-A \\
\hline Total GRAS & $-0.13^{*}$ \\
\hline
\end{tabular}

$N=221$

* $\mathrm{p}<.015$

Correlation was weak and negative $(r=-0.13)$ at $p<.05$ significance level. Calculated for only female students correlation was weak $(r=-0.19)$ and significant $(p<.05)$ and for only male students somewhat stronger $(r=-0.30)$ and significant again $(p<.01)$. Pearson's $r$ calculated for School of Business students was not significant $(r=-0.09), p=.39)$ but correlation coefficient for School of Social Work students was significant again $(r=-0.26, p=.01)$.

In order to further investigate this finding, Total A.S.A.-A scores were correlated with the individual adjectives of the G.R.A.S. . Results are given in Table 5.24. The highest positive Pearson's correlation coefficients were calculated for the adjectives 'Attentive' $(r=.35)$, "Well-balanced' $(r=.23)$, 'Selfconfident' $(r=.22)$ and 'Self-assured' $(r=.21)$. These were all significant at $p<$ .001 level. Total A.S.A.-A scores were also positively correlated to the adjectives 'Inventive' $(r=.19)$, 'Highly-gifted' $(r=.19)$, 'Alert' $(r=.18)$, 
'Radical' $(r=.18)$, 'Sensual' $(r=.18)$ and 'Dominant' $(r=.17)$ all at a $p<.01$ significance level and 'Original' $(r=.16)$ 'Diligent' $(r=.14)$ and 'Firm' $(r=.13)$ at $p<.05$ levell. Significant were also the following negative correlations. The higher college students' Total A.S.A.-A score the less they tended to perceive themselves as 'Un-balanced' $(r=-0.27, p<.001)$, 'Undecided' $(r=-0.24, p<$

Table 5.24 Correlations Total A.S.A.A Items G.R.A.S.

\begin{tabular}{|c|c|}
\hline Iters GRAS & Total ASA-A \\
\hline Attentive & $.35 *$ \\
\hline Un-balanced & $-0.27 *$ \\
\hline Undecided & -0.24 \\
\hline Well-balanced & $.23^{*-*}$ \\
\hline Self-confident & $.22 k *$ \\
\hline Self-assured & $.21 *$ \\
\hline Uncertain & $-0.21 *$ \\
\hline Nervous & $-0.20 *$ \\
\hline Inventive & $.19 *$ \\
\hline Highly gafted & $.19 * 4$ \\
\hline Radical & .18 th \\
\hline Alert & $.18 *$ \\
\hline Sensual & $.18 * *$ \\
\hline Dominant & $.17 * *$ \\
\hline Orïginal & $.16 *$ \\
\hline Jumpy & -0.16 \\
\hline Touchy & $-0.16 *$ \\
\hline Feminine & .15 \\
\hline Materialistic & $-0.15^{*}$ \\
\hline Shy & $-0.15^{*}$ \\
\hline Dilligent & $.14^{*}$ \\
\hline Fürm & $.13^{*}$ \\
\hline Individualistac & $-0.13^{*}$ \\
\hline Timid & $-0.13^{*}$ \\
\hline
\end{tabular}

$$
\begin{array}{rl}
* p & <.05 \\
* & p<.01 \\
* * * & <<.001 \\
N & =.223
\end{array}
$$


.001) 'Un-certain' $(r=-0.21, p<.01)$, 'Nervous' $(r=-0.20, p<.01)$, 'Jumpy' $(r$ $=-0.16, p<.05)$, 'Touchy' $(r=-0.16, p<.05)$, 'Shy' $(r=-0.15, p<.05)$, 'Materialistic' $(r=-.15, p<.05)$, 'Individualistic' $(r=-0.13, p<.05)$ and 'Timid' $(r=-0.13, p<.05)$. Total A.S.A.-A scores were not significantly correlated with college students' qualification of themselves as 'Masculine'. On the other hand the higher A.S.A.-A scores the more subjects tended to perceive themselves as 'Feminine' $(r=.15, p<.05)$.

The above given results demonstrate empirical evidence that operability of Self-Care Agency was weakly but significantly related to sex-stereotyped selfimage. This was the case for all college students taken together as well as for male and female students seperately and for School of Social Work students. In this respect an exception was made by School of Business students. Significant correlations were also demonstrated for G.R.A.S.-adjectives and Total A.S.A.-A scores. Total A.S.A.-A scores had highest positive correlations with the adjectives 'Attentive', 'Well-balanced', 'Self-confident' and 'Self-assured' and highest negative correlations with 'Unbalanced', 'Undecided' and 'Uncertain'. Total A.S.A.-A was significantly correlated with the adjective 'Feminine' and not with 'Masculine'. In combination the above results can be seen as supportive of hypothesis twenty.

Hypothesis twenty-one predicts a significant negative correlation between Total A.S.A.-A scores and age for subjects older than 65 years. Data were used of the combined studies I till VI. A Total of 275 subjects participating in the studies of the research program were 65 years or older. Table 5.25 gives the calculated Pearson's correlation coefficient.

Table 5.25 Correlation Tolal A.S.A.-A. and Age (> 65 years)

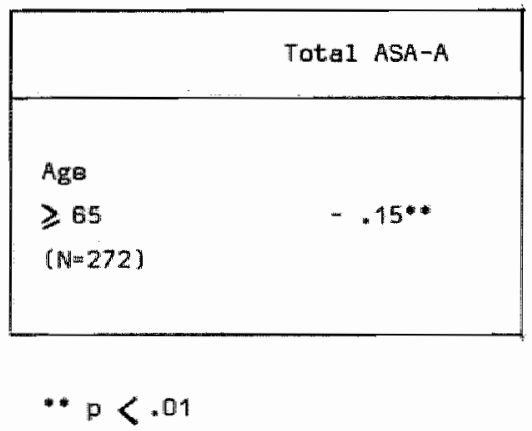

Pearson's $\mathrm{r}$ is negative and significant at $p<.01$ ) level. Correlation coefficient between age and Total A.S.A.-A was also calculated for all subjects of the research program and was not significant $r=.03, p=N . S$., $n=672$. This was 
also the case for correlation coefficient calculated for all subjects in studies I-VI together $r=.03, p=\mathrm{N} . \mathrm{S} ., \mathrm{n}=449$ and in study VII $r=.09, p=\mathrm{N} . \mathrm{S}, \mathrm{n}=223$. Hypothesis twenty-one is supported by these results.

In order to further investigate the relation of Total A.S.A.-A and age for all subjects of the research program the following age categories were formed: $18-24(n=217), 25-54(n=61), 55-64(n=122), 65-74(n=122), 75-84(n=111)$ and 85-97 $(n=39)$. The categories $25-34,35-44$ and $45-54$ were taken together because of the few number of subjects represented. This was also the case with categories 85-94 and $\geq 95$. Difference in mean Total A.S.A.-A scores of these age groups was tested by ANOVA and found to be significant $F(5)=5.73, p=$ .02. Figure 5.2. shows the mean Total A.S.A.-A scores for the different age-groups.

Figure 5.2. $\bar{x}$ Total A.S.A.-A scorest for different age-groups

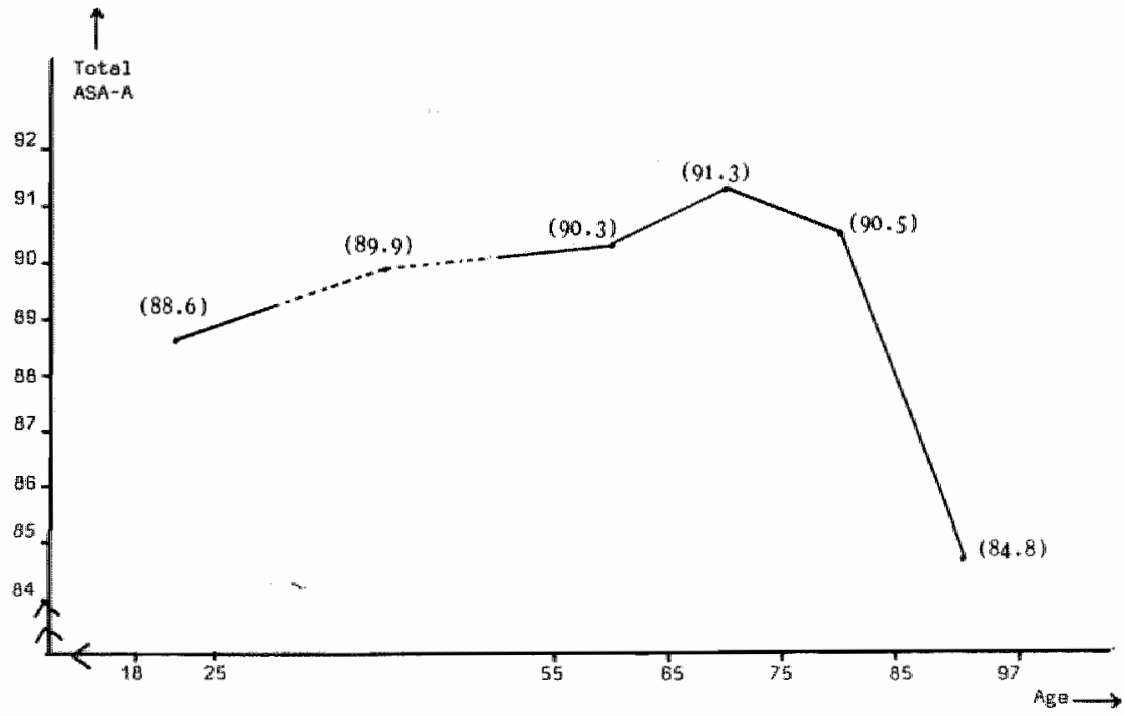

Figure 5.2. shows a curvilinear pattern with mean Total A.S.A.-A scores gradually increasing for age-groups till the 65-74 group and than clearly decreasing. This is consistent with Orem's theory, which states that Self-Care Agency begins to develop in childhood and continues to increase during adulthood and declines with advancing age. Orem states that it is in the course of day to day living through the spontaneous process of learning that Self-Care Agency 
develops. Its development is aided by intellectuall curiosity, by instruction and supervision from others and by experience in performing Self-Care measures (Orem, 1980). Given a degree of development Self-Care Agency can be more or less operative in points of time. Operability is affected by different factors one of these being health state (Orem, 1980). Eta calculated for the total sample of the research program was $\eta=.14$. Eta squared was $\eta^{2}=.02$ indicating that in this sample only $2 \%$ of Total A.S.A.-A score variance was explained by age difference. When subjects without patient status $(n=293)$ were taken apart squared eta calculated for this group increased to $\eta^{2}=.22, p<.001$.

\section{Factor-analysis}

Factor-analysis for construct validity testing was exploratory and to a certain extent also confirmatory in nature. This purpose was different from the one earlier described within the context of reliability testing. Factor-analysis was done on first measurement A.S.A.-A scores of all subjects of the total sample of the research program (study I-VII) and on A.S.A.-A scores of the subsample of predominantly older subjects with health problems (study I-VI) and of the subsample of predominantly young healthy subjects (study VII). Principal components method was used for factor extraction. Cut off point for factor extraction was established at eigenvalues $<1.00$. On data of study I-VII and I-VI 7 factors were extracted explaining $53 \%$ and $55 \%$ of total variance. Eight factors were extracted in study VII explaining $58 \%$ of total variance. Orthogonal factor rotation was performed. Items loading on the seven respectively eight factors are given in Annex I Table 1 with factor loadings and communalities. Factor eight in study VII contains only one item: nr. 8 'Adjusting bathing frequency'. To analyse factor structure robustness across the samples of study I-VII, study I-VI and study VII the item numbers, related Self-Care Agency Power Components and nature of the items loading on the seven respectively eight factors were compared. Annex I Table 2 gives the detailed results. There was no factor consistently containing all positive or all negative items. There was only one item number consistently represented in the same factor: nr. 3 'Making mobility related adjustments' in factor 1. It's related Self-Care Agency - Power Component is III 'Movement'. Two more Self-Care Agency - Power Components were consistently represented in two factors: in the third factor P.C. IX 'Priority setting' and in factor four P.C. VII 'Knowledge'. When factor three of study VII was compared to the second factor of study I-VII and I-VI two out of three items had the same number and related power component: item nr. 23 "Having time for self', P.C. IX 'Priority setting" and item nr. 20 'Taking time for self-Care'. P.C. VI 'Decision making'. The third item in factor two in study I-VII and I-VI was nr. 11 'Sufficient daily rest and exercise". The third item in factor three of study VII was nr. 10 'Managing to be alone'. Annex I Table 3 shows the distribution of all empirical referents across the factors in study I-VII, I-VI and VII. In conclusion comparison of seven or eight extracted 
Table 5.26 them Number, Nature and Self-Care Agency-Power Components of 2 Factors of A.S.A.A

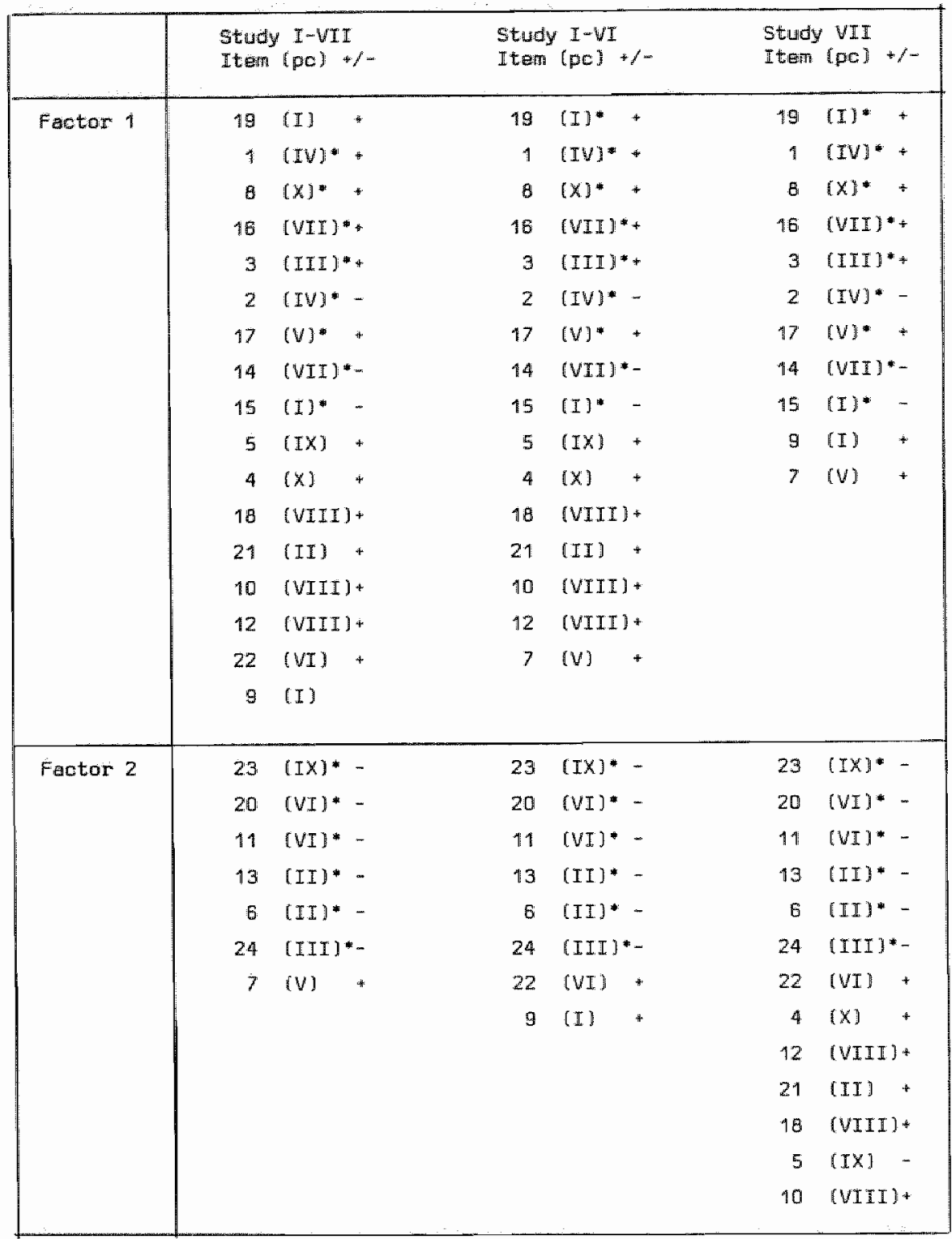

factors of the rotated matrix of factor anallysis of A.S.A.-A scores with SelfCare Agency - Power Components did not show a convincing and consistent pattern of clustered Self-Care Agency-Power Components. 
In order to further explore the relationship between clusters of Power Components and factor structure a more strict criterium was chosen to establish a cut off point for factor extraction. Based on the principle of discontinuity a clear drop in the percentage of explained variance is suggested as an appropriate termination point (Kim, Mueller, 1982; Polit and Hungler, 1987). After the second factor in the analysis of study I-VII, of study VII the difference of explained variances of successive factors is relatively small and less than the difference between factor two and three. Thus two factors were extracted factoranalysing the A.S.A.-A scores of the three samples again. Annex II Table 1 gives the items loading on the two factors with related factor loadings and communalities. Factor structure robustness across the samples was analysed again by comparing item numbers, related Self-Care Agency - Power Components and nature of items loading on the two factors. Table 5.26 shows results. No one of the two factors consistently contained all positive or all negative items. In the first factor nine items were consistently represented across the three samples. They are marked with an * in table 5.26. Number 19 'Effectiveness evaluation', nr. 1 'Making adjustments', nr. 8 Adjusting bathing frequency', nr. 16 'Obtaining medication related information', nr. 3 'Making mobility related adjustments', mr. 2 'Checking measures', nr. 17 'Changing old habits', nr. 14 'Asking for clarification', nr. 15 'Body examination'. In the second factor six items consistently were represented across the three samples. The items nr. 23 'Having time for self', nr. 20 'Taking time for self care, nr. 11 'Sufficient daily rest and exercise', nr. 13 'Getting enough sleep', nr. 6 'Sufficient energy', and nr. 24 'No mobility limitation'.

Within this robust factor structure the pattern of Self-Care Agency - Power Component clusters of the two factors was explored. The criterium of at least two of the same Self-Care Agency-Power Components represented per factor was used for further item analysis. Results are given in table 5.27.

Table 5.27 shows that Power Components I, IV, VII are consistently represented in Factor 1. Attention, Reasoning and Knowledge cluster together. Motivation, skills and Integration alternate in addition. Power Components II and VI consistently are represented in factor 2 . Energy and decision making cluster together while skills and priority setting alternate in addition. Power Component III movement is not represented in this pattern. In addition internal and external consistency of factor structure was analysed. Cronbach's alpha's were calculated for both factors on measurements in the three samples. The alpha (standardized) of factor one was in study I-VII, .75, in study I-VI, 78 and in study VII .71. The alpha's for factor two were respectively $.47, .59$ and .70. Correlation coefficients were also calculated between factor one and two. Pearson's $r$ 's were all significantly positive. $r=.35, p<.001$ in data of study I-VII, $r=.21, p<.001$ in study I-VI and $r=.31, p<.001$ in study VII. These results demonstrate coherent and related substructure of two factors and a 
Table 5.27 Self-Care Agency Power Component - Clusters in 2 Factors of A.S.A. «A

\begin{tabular}{|c|c|c|c|c|c|c|}
\hline & Stud & $\begin{array}{l}\mathrm{I}-\mathrm{VII} \\
{ }^{*} \mathrm{C}\end{array}$ & Stud & $\begin{array}{l}I-V I \\
\text { P.C. }\end{array}$ & study & $\begin{array}{l}\text { VII } \\
\text { P.C. }\end{array}$ \\
\hline \multirow[t]{6}{*}{ Factor 1} & $x$ & At tention & 1 & Attention & $\mathbb{I}$ & Atrention \\
\hline & IV & Reasoning & IV & Reasoning & IV & Reasoning \\
\hline & VII & Knowledge & VII & Knowledge & VII & Knowledge \\
\hline & & & $v$ & Motivation & $\mathrm{v}$ & Motivation \\
\hline & VIII & Skills & VIII & Sk+1118 & & \\
\hline & $x$ & Integration & $\mathrm{x}$ & Integration & & \\
\hline \multirow[t]{6}{*}{ Factor 2} & II & Energy & II & Energy & II & Energy \\
\hline & VI & Decision & VI & Dectsion & VI & Dectsion \\
\hline & & Mak1ng & & Makfing & & Maklng \\
\hline & & & & & VIII & Skills \\
\hline & & & & & $\mathbb{E X}$ & Priority \\
\hline & & & & & & Setting \\
\hline
\end{tabular}

consistent relationship between factor substructure and clusters of Self-Care Agency - Power Components. As such factor analysis results are supportive of construct validity of the A.S.A.-A scale.

\subsection{Measurement Conditions}

Hypothesis twenty two predicts no significant positive correlation between CABS patients' Total A.S.A.-A scores and their Total scores on the adapted C.

Table 5.28 Correlation Total A.S.A.A - Social Desirability

\begin{tabular}{|c|c|}
\hline & Tota 1 ASA-A \\
\hline 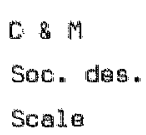 & $\cdot 14^{\mathrm{ng}}$ \\
\hline
\end{tabular}

$$
\mathrm{nis}=\text { mot significant } \quad N=90
$$


\& M. Social Desirability scale. A significant positive correlation would be indicative for response set bias. Data were used of six months post-operative measurements of study VI: CABS. Table 5.28 gives the results.

Pearson's $\mathrm{r}$ is positive but not significant (one tailed) at $p<.05$ level calculated for all 98 patients. Social desirability was not significantly correlated with age or years of formal education, but female patients had a significantly higher score on the C. \& M. Social Desirability scale $\overline{\mathrm{x}}=64.3, \mathrm{SD}=7.2, \mathrm{n}=22$ than male patients $\overline{\mathrm{x}}=58.1, \mathrm{SD}=8.5, \mathrm{n}=76, T(96)=3.09, p=.003$. Pearson's correlation coefficient calculated between Total A.S.A.-A and Social Desirability for female patients was positive and somewhat stronger $r=.28$ but not significant (one tailed) at $p<.05$ level. Pearson's $\mathrm{r}$ for male patients was $r=.09, p=$ N.S. Results are supportive of hypothesis twenty two.

The last hypothesis formulated in chapter three is number twenty three. This hypothesis predicts, that mean Total A.S.A.-A score of CABS-patients to whom the scale is personally administered twelve months post-operative will not significantly differ from the mean Total A.S.A.-A score of comparable patients to whom the scale is send by mail. No significant difference indicates absence of measurement error in this respect. Data were used of twelve months measurements of study VI: CABS. Results are given in table 5.29.

Table 5.29 Difference score Total A.S.A.-A Mailed and Personally Administered

\begin{tabular}{|c|c|c|c|}
\hline & $\bar{x}$ & $\mathrm{SD}$ & $t$-value (df) \\
\hline$A S A-A$ scale matled $(N=35)$ & 92.91 & 7.64 & \multirow{3}{*}{$0.75^{\mathrm{ns}}(95)$} \\
\hline ASA-A scale personally & 91.42 & 10.28 & \\
\hline administrated $(N=62)$ & & & \\
\hline
\end{tabular}

$n B_{1}=$ not significant

Mean Total A.S.A.-A score of patients to whom the scale was send was 92.9 and somewhat higher than mean Total A.S.A.-A score of patients to whom the scale was personally administered $\bar{x}=91.4$. The difference was not significant (two tailed). Standard deviation of Total scores of the mailed A.S.A.-A was lower than SD of scores of the personally administered A.S.A.-A. The difference was not significant $F=1.81, p=\mathrm{N}$.S. Results are supportive of hypothesis twenty three. No evidence of measurement error in this respect was demonstrated. 


\section{DISCUSSION AND CONCLUSIONS}

\section{Introduction}

This chapter summarizes and discusses the main findings reported in chapter five. Congruent with the purpose of this study conclusions are formulated in terms of reliability and validity of the A.S.A.-A scalle. Methodological limitations are discussed in relation to the program of research described in the fourth chapter. Theoretical implications are set fourth in relation to Orem's Self-Care Deficit Theory in particular and the need for theory testing in general. The chapter ends with implications of this study for nursing practice.

\subsection{Reliability}

In terms of internal consistency of the A.S.A.-A scale Cronbach's alpha was used to quantify the relative absence of error variance. Calculated for the first measurement of A.S.A.-A with all 676 subjects in the program of research alpha was .76. A.S.A.-A scales were self-administered, used in standardized interviews and mailed. Measurements were done with healthy subjects and patients in age ranging from young adults to elderly "Measurement locations were at home and in a variety of health care institutions. The first hypothesis was consistently supported across all measurements. All alpha's with the exeption of two borderline cases of . 69 were higher than .70. This was even so when measurement was reallised with anxious patients shortly before a major life threatening surgical procedure. For making group level comparisons alpha's ranging from $.60-.70$ were suggested to be sufficient. However if scores are used as a basis for making decisions about individuals reliability coefficients should be arond .90 . As stated earlier, reliability and validity are matters of degree. Resullts in this study demonstrate that A.S.A.-A can be used in an acceptable way as a reliable, internal consistent instrument for group level comparison of adult healthy subjects and patients in health care institutions or at home even under extreme measurement conditions. Caution should be taken, when the purpose is a decision about an individual.

Presupposed unidimensionality of the A.S.A.-A scale as a summative scale of a Likert type was tested by hypotheses two till five. For this purpose all items 
were analysed in respect to whether they significantly differentiated between low and high Total A.S.A.-A scores. Calculations were compared for the entire sample of the research program and for the subsamples of predominantly older subjects with health problems and predominantly younger healthy subjects in order to validate stability of results. Hypothesis three was strongly supported for all 24 items across the three samples. As such this result is indicative for unidimensionality or homogeneity of the instrument. Unidimensionality was also tested by comparing the percentage of explained variance of the first factor with subseguent factors extracted from the unrotated factor analysis of the A.S.A.-A scale. Hypothesis four was not supported. The first factor explained 1.83 and not 2 times the variance of the second factor. Though not as large as expected, the difference between explained variance of the first and subsequent factors is substantial. As such this result indicates the presence of one clean first factor. Subsequent factors explained fairly equal proportions of the remaining variance with a gradual decrease. It is only after factor two that this decrease is consistently gradual. This may indicate the possible existence of a less clear second factor.

Hypotheses two and five tested item to total correlations $(\geq .30)$ and itemloadings on the first extracted factor of the unrotated factor analysis $(\geq .35)$ of all 24 items of the A.S.A.-A scale as criterion measure for unidimensionality. The second hypothesis was consistently supported for 11 items, partly for 7 items and consistently not supported for 6 items: Hypothesis five was consistently supported for 13 items, partly for 8 items and consistently not supported for 3 items. Consistent support across all six calculations done for hypothesis two and five means strong contribution to unidimensionality. This is the case with 11 items: $\mathrm{nr} .1, \mathrm{nr} .2$, nr.3, nr.4, $\mathrm{nr} .14$, nr.15, nr.16, nr.18, nr.19, nr.20, nr.21. When Self-Care Agency - Power Components of these 11 items are compared three out of ten are not represented: P.C. II: energy, P.C. V: motivation, and P.C. IX priority-setting. When compared with the two factor structure presented in Table 5.26 (chapter 5) items nr.1, 2, 3, 14, 15, 16 and 19 are consistentlly represented in factor one, item 20 consistently in factor two and the items nr.4, 18 and 21 fluctuate across the two factors. Reduction of the A.S.A.-A scale to 11 items based on these very strict criteria would seriously affect construct validity of the scale. The pattern of Self-Care Agency - Power Component clusters as presented in Table 5.27 in chapter five would be affected especially in terms of P.C. V motivation and X integration in the clusters of factor one and in terms of P.C. II energy, VI decision making and IX priority setting in factor two. The remaining cluster of power components would be I attention, IV reasoning, VII knowledge and VIII skills. Reduction of the scale to these 11 items would not affect reliability coefficient substantially: alpha $=$ .72 . Result of such reduction would be a reliable instrument measuring only a certain part of the Self-Care Agency construct.

Not consistent but partly support for hypotheses two and five may be indicati- 
ve that the item concerned is sensitive for typical characteristics of the two subsamples i.e. age and health status. This may have been the case with for instance item nr.19 'I eat in a way that maintains my body weight at an appropriate level". This item had a low item to total correlation and a low loading on the first factor in the sample of older subjects with health problems. On the other hand the same item had a high item to total correlation and a high loading on the first factor in the sample of young healthy adults. This item obviously has a different meaning for these two different cultural groups in the context of Self-Care Agency. A similar argumentation could be given for item nr. 22 'I seek help when unable to care for myself' and other partly supported items. Thus for reasons of 'cross-cultural' comparability such items should be retained. Items for which hypotheses two and five consistently are not supported in this study seem not to contribute to unidimensionality of the instrument. As such they could be dropped to increase reliability of the instrument. When results of hypotheses two and five are compared three such items can be identified: item nr.7 'seeking improvement', nr.12 'having a circle of friends' and nr.24 'no mobility limitations'. When power components of these items are compared (P.C. V, VIII, and III) it is clear that dropping these items does not influence representation of all Power Components in the 21 item version of A.S.A.-A. Nor does it affect the pattern of Self-Care Agency - Power Component clusters as presented in Table 5.27 in chapter five. In combination results for hypotheses two till five demonstrate unidimensionality of the A.S.A.-A scalle. Homogeneity can be improved without affecting construct validity by dropping items nr.7,21 and 24. The question whether the instrument contains more than one factor will be discussed within the context of construct validity.

In terms of precision of the instrument the following findings were important. The shape of the frequency distribution of first Total A.S.A.-A scores of all subjects participating in this research program was unimodal and negatively skewed. Within the subsample of predominantly young healthy subjects frequency distribution shape was less skewed, more normal. This is congruent with the expectation for such a 'normal' population sample. When skewness was analysed at item level nr.4 was the only item showing extremely skewed response frequencies consistently across all studies. Ninety to even $100 \%$ of respondents did agree with this statement of operability of Self-Care Agency as being reflective of themselves. As such this item was not sensitive enough in this study and for reasons of parsimony it can be dropped from the Dutch version of the A.S.A.-A scale.

The empirical range of all Total A.S.A.-A scores was in between 37 and 117 . Theoretically scores could range from $24-120$. Only a small percentage of Total A.S.A.-A scores was lower than 60 in this sample. The difference between lowest and highest empirical mean score was 20.3 points in the study with an elderly population and 4.9 points in the other combined studies. It can be 
concluded that values of all scores along the range are systematically inflated, but that collapsing the range of empirical scores would affect precision of measurement. In order to produce more meaningfull scores they can be lowered leaving the empirical range between highest and lowest score as it is. This can be realised by changing the score values associated with Totally Disagree to Totally Agree from $1-5$ into $0-4$. This results in a theoretical Total score range from 0 - 96 for the 24 item A.S.A.-A version and from $0-80$ for the 20 item A.S.A.-A version reduced based on the before mentioned conclusions.

Equivalence was tested by two hypotheses predicting a significantly positive and weak to moderate correlation between subjects' Total A.S.A.-A scores and their Total A.S.A.-B scores given by significant others (H.6) and by nurses (H.7). Hypothesis six was supported by calculation done on data of the total sample. Results were consistent for most but not all subsamples. One exception was found in Study IV HOP. Correlation was slightly stronger $r=.72$ than expected. Another exception was found for 23 patients in a physical rehabilitation center. Correlation was positive $r=.32$ but not significant. The small $\mathrm{n}$ of this sample in relation to the other samples may have influenced non-significance. Apart from this statistical explanation a more substantial one is also possible. Patients were hospitalized in the center for a physical handicap developed after a recent dramatic change in health state like a Cerebro Vascular Accident and Spiral Cord Leasion. Patients lived separated from their significant others for longer periods. The lower correlation coefficient in this study compared to the others may be attributed to incongruence between patients and their significant others in perception of change in Self-Care Agency after dramatic change in health state. Consistent with this explanation is the finding of no significant and even a weak negative correlation between these patients' Total A.S.A.-A scores and their Total A.S.A.-B scores given by nurses (H.7). Validity of patients' perception of their Self-Care Agency may be at least temporarily questioned under a condition of important negative change in Self-Care Agency due to acute health state related Self-Care limitations or important positive change in Self-Care Agency due to development of effective alternative Self-Care Strategies. The other non-significant but positive $(r=.18)$ correlation in relation to hypothesis seven was calculated for reumatoid arthritic patients living at home and their community nurses. This lack of significance may again be statistically explained by the small $n$ (21). Furthermore within this group community nurses who were only visiting the patient on a bimonthly or even yearly basis were also represented. Diversity in human interaction and communication between patient and nurse may also have contributed to the though significantly positive but still weak $(r=.28)$ correlation found in the Nursing Home study. In this respect Study IV HOP can be considered the opposite case. Study design was such, that nurses in the acute care hospital were explicitly asked to select those patients who they knew best. As such a more standardized and more optimal situation of human communication was artifi- 
cially created. This may explain why positive correllation in this study was both significant and the highest $(\mathrm{r}=.56)$ in all studies. As such a not unimportant contribution was made by this study to the significantly and moderately positive correlation calculated on combined data of studies I till IV to test hypothesis seven. Results demonstrate that self-appraisal A.S.A. scores cannot be considered identical to appraisal of another A.S.A. scores. On the other hand sufficient evidence was gathered that Total A.S.A.-A and Total A.S.A.-B can be considered equivalent measures in general. This may not hold under conditions of important negative or positive change in Self-Care Agency or conditions of less or lack of human interaction and communication.

Stability was tested for a six weeks (H.8) and six months (H.9) time-interval. Both hypotheses were strongly supported. Correlation coefficient was lower for the six months than six weeks time interval. This may indicate that Self-Care Agency is less permanent across longer periods of time. Due to the relatively small $\mathrm{n}$ in the six weeks study analysis for subsamples of low, medium and high scores was only done for the six month interval. Low scores were not significantly and lowest correlated with a significant increase in mean total score. Medium scores were significantly moderate and positively correlated and showed no significant change in mean score. High scores were positively but not significantly correlated and showed no significant change in mean score. Lack of significant correlation and significant increase for low scoring coronary artery bypass patients at six and twelve months may indicate, that these patients experience the first six months post-operative improvement (see Table 5.20, chapter five) later. This would indicate that low scorers are slow and less predictable Self-Care Agency improvers than medium and high scorers. Lack of significant correlation and change in mean score for high scorers may indicate that above a certain point of operability of Self-Care Agency it is difficult to consistently differentiate between a high and very high degree of operability. Such non significance could be considered as an artefact due to insufficient measurement precision. The difference in range of scores between medium ( $\geq$ $88 \leq 95)$ and low $(<88)$ and high $(>95)$ may also have influenced strength or significance of correlations. Results demonstrate that stability or permanence in Self-Care Agency can be measured with A.S.A.-A. Permanence of Self-Care Agency measured by stability in A.S.A.-A scores seems to be dependent from change in health state, from level of operability of Self-Care Agency and the time-interval between measurements.

In conclusion. The A.S.A.-A scale can be used as a reliable, internal consistent instrument for group level comparisons of adult healthy subjects and patients at home and in health care institutions even under extreme measurement conditions. The scale should be used with caution for decisions about an individual. Self-Care Agency is unidimensionally measured with this scale. Differences across cultures influence homogeneity of A.S.A.-A measure- 
ments. Unidimensionality of the Dutch version of A.S.A.-A can be improved by dropping items 7,12 and 24 . For reasons of parsimony item nr. 4 can also be dropped. Precision of measurement of very high levels of operability of SelfCare Agency with the A.S.A.-A scale may need improvement. More meaningfull Total A.S.A.-A scores are obtained when individuall item score possibilities are changed from 1-5 into 0-4. Self appraisal A.S.A.-A scores are not identical but in general equivalent to appraisal of another scores. This is at least not the case under conditions of important negative or positive change in Self-Care Agency or when human interaction and communication is decreased or absent. Permanence or stability in Self-Care Agency can be measured with A.S.A.-A but is dependent from change in health state, levell of operability of Self-Care Agency and the time interval between measurements.

\subsection{Construct Validity}

In terms of construct validity the A.S.A.-A scale was tested for its possibility to discriminate significantly the level of mean scores of four groups of elderly known to differ in actual dependency on institutionalized nursing and related care. A negative relationship was assumed: the higher operability of Self-Care Agency the less dependent on institutionalized nursing and related care. Elderly living independently in the community scored significantly higher in operability of Self-Care Agency than service flat residents (H.10), who on their term scored significantly higher than personalized care facility residents (H.11). Nursing home residents scored lowest, but the difference with personalized care facility residents was not significant (H.12). Non significance may be caused by some underrepresentation of the very ill e.g. terminal and (sub) comateuse patients. Important to note in this respects is that also all psychogeriatric patients were excluded from the nursing home sample. On the other hand non significance may indicate that the difference in Self-Care Agency of nursing home patients and personalized care facility residents is not great. Remmen (1985) in his study could not demonstrate an important difference from a medical perspective between a nursing home population and personalized care facility residents. Based on study designs his results can be more confidently generalized than this study's finding in this respect. The difference of nursing home patients' Self-Care Agency compared with SelfCare Agency of the other groups of elderly was significant. The difference stayed significant when scores were standardized for age and sex. Differences in operability of Self-Care Agency between groups of elderly in various degrees of dependency on nursing care can accurately be demonstrated in the predicted direction using the A.S.A.-A scale.

Operability of Self-Care Agency of patients of a physical rehabilitation center measured with A.S.A.-A correlated significantly positive with measurement results on the short version of Kearney and Fleischer's Exercise of Self 
Care Agency ESCA-scale. This version is supposed to reflect certain Power Components and Self-Care Operations of the concept Self-Care Agency as defined by Orem. Correlation between A.S.A.-A and the 16 item version of ESCA was moderate $(r=.48)$ and not strong as expected. On the one hand this may indicate, that this shortened version of ESCA does indeed measure some Power Components and Self-Care Operations as suggested by Isenberg (1986). On the other hand as A.S.A.-A was develloped based on analysis of Orem's and N.D.C.G.'s description of the Self-Care Agency concept with the explicit attempt to cover all Power Components and productive Self-Care Operations, it may be assumed that the short version of the ESCA scalle doesn't cover the content of Orem's Self-Care Agency concept as well as the A.S.A.-A scale. Nevertheless as expected results indicate that measurement of operability of Self-Care Agency with A.S.A.-A converges with the ESCA scale. Measurement of operability of Self-Care Agency with A.S.A.-A also converged with responses on the one sentence question: 'How do you assess your capability to care for yourself. Possibilities to answer ranged from very bad to very good. Correlation was significantly positive and weak $<.40$ for the population of the research program as a whole. This was expected (H.14) because a simple item cannot fully represent a complex theoretical concept, lacks precision and is more prone to random error than a multi-item scale. Apart from this psychometric explanation it is also important to realise that the one sentence question may have been interpreted as asking for adequacy of Self-Care Agency while A.S.A.-A reflects operability. This could explain why this sentence did not correlate significantly with A.S.A.-A for the group of chromic, reumatoid arthritic patients staying at home (study II: RAP). These patients experiencing important movement limitations may have explicitly interpreted the one sentence statement as asking for adequacy. In that case operability of Self-Care Agency in general may not have been directly related to adequacy because of interfering movement limitations. With only this one exception all correlations between A.S.A.-A and this one sentence question were consistently significant and positive across the different studies. This convergence can also be considered as evidence for construct validity of A.S.A.-A.

On the other hand the concept of Self-Care Agency as measured by A.S.A.-A could also empirically be discriminated from the Activities of Daily Living A.D.L.-concept (H.15). This was tested with chronic reumatoid arthritic patients and older patients recently discharged from a hospital. No significant correlation was found between reumatoid arthritic patients' Total A.S.A.-A scores and their Total A.D.L.-scores given by significant others and community nurses. No significant correlation was found between older discharged patients' Total A.S.A.-A scores and their self-appraisal Total A.D.L. scores. This was consistent with expectations. Theoretically both concepts stand for different idea's: A.D.L. refers to performance, Self-Care Agency to capability to decide what needs, can and will be done and to actually realise 
change to fullfill eight universal and some developmental and health related needs for self care; A.D.L. refers to degree of independent functioning while Self-Care Agency is assessed on operability with A.S.A.-A. Total A.S.A.A was significantly negatively correlated with two individual items of the A,D L.observation of the reumatoid arthritic patients done by their significant others. The lower the operability of Self-Care Agency of these patients the more dependent they were on assistance with feeding and toiletting according to their significant others. Need for assistance with feeding and toiletting may be seen as related to mobility limitations of these patients, which on its term are related to reumatoid arthritic disease. No significant correlations were found between Total A.S.A.-A scores and individual A.D.L. item scores of older patients two weeks after hospital discharge. Both self-assessment of Total A.D.L. as well as assessment by others i.e. family and nurses were not related to Self-Care Agency. The demonstrated possibility to empirically discriminate Self-Care Agency from A.D.L. adds evidence to construct validity of A.S.A.-A.

Self-Care Agency within Orem's Self-Care Deficit theory is related to SelfCare in such a way, that persons who take action to provide their own Self-Care have Self-Care Agency. Coronary artery bypass surgery patients' Total A.S.A.-A scores in study VI were significantly positively correlated with their Total R.P.R.I. scores (H.16). This finding was consistent at pre-operative measurement and at six and twelve months post-operative measurements. This indicates that the more operable these patients' Self-Care Agency was the more adequate their self-care in relation to universal requisites. It seems logical but because of limitations of the design not necessary to assume that operability of Self-Care Agency was antecedent to adequacy of Self-Care. The consistency in the pre- and post-operative findings indicate that the positive relation holds both for states of bad and good health. Correlations were weak ranging from .19 to .24 . This indicates that the concept of self-care can be empirically discriminated from the concept of Self-Care Agency. It should also be realised, that transition of capability into actual performance in general is influenced by variables like environmental conditions, availability of resources, tools e.a. In this study, operability of Self-Care Agency was related to adequacy of universal self-care. More operable Self-Care Agency may but not necessarity has to lead to more adequate Self-Care in relation to Universal Requisites. For reasons of construct validity it can be concluded, that the proposed relationship of SelfCare Agency and Self-Care can be demonstrated with the A.S.A.-A scale in situations of bad and good health.

The theoretically proposed relationship health and Self-Care Agency was also tested. Coronary artery bypass patients' pre-operative mean Total A.S.A.-A score was significantly lower than their six months post-operative mean score (H.17) and also compared with their twelve months mean score (H.18). No significant difference was found between mean six months and 
mean twelve months score. This was important because no control group A.S.A.-A scores were available. The findings support the idea, that positive change in Self-Care Agency was the effect of change in health state related to cardiac revascularisation. As such this means strong evidence for construct validity of A.S.A.-A.

Operability of Self-Care Agency according to Orem is positively affected by learning capabilities, orientation, self-understanding, self-awareness, self-value and self-acceptance. These basic capabilities and dispositions on their term are positively influenced by an individual's (sub)iculture in which social support is highly valued and practiced. Chronic reumatoid arthritic patients' Total A.S.A.-A scores were significantly positively correlated with Total Functional Social Support N.S.S.Q. scores as well as with Total Network N.S.S.Q. scores. The larger the patients' social network, the larger the length of time of contacts, the more patients felt respected and supported the more operable their SelfCare Agency was. This finding is congruent with Orem's theoretical notions. The correlational nature of this study design empirically leaves the following question unanswered. Did more perceived social support with these chronic patients at home increase operability of their Self-Care Agency or did more operable Self-Care Agency cause them to increase their (perceived) social support. Theoretically a third unknown variable may have caused the positive relation between the two. In terms of construct validity it can be concluded that consistent with the theory a significant positive relation between social support and Self-Care Agency can be demonstrated with A.S.A.-A.

One of the dispositions theoretically proposed to affect Self-Care Agency is self-image. College students' sex-stereotyped self-image indeed correlated significantly with Self-Care Agency as measured with A.S.A.-A. This finding was consistent for male and female students and for School of Social Work students, but not for School of Business students. These students self-image was most masculine. This may indicate that extreme masculine self-image does not influence operability of Self-Care Agency. Only a weak correlation was expected and found with sex-typed self-image as both stereotype male (active, rational) and stereotype female (caring) qualities are required for Self-Care Agency. Significant positive correlations were demonstrated for adjectives reflective of both stereotype masculine and feminine qualities. Most items which correlated most strongly with Total A.S.A.-A could be related to the notion of deliberate action. This is consistent with Orem's definition of SelfCare Agency. On the other hand Total A.S.A.-A was also significantly correlated to the adjective 'feminine' and not to 'masculine'. Thus the more attentive, well-balanced, self-confident, self-assured, decided, certain and feminine, students qualified themselves the higher their operability of Self-Care Agency as measured with A.S.A.-A. As such this can be considered evidence again for construct validity of A.S.A.-A. 
Operability of Self-Care Agency was negatively correlated with age for persons of 65 years or older. This indicates that for elderly operability of Self-Care Agency decreases with increasing age. Decrease and increase are not strongly correlated. In fact in this study operability of Self-Care Agency decreased strongly only in the age group of 85 years of older. A curvilinear pattern could be demonstrated between Self-Care Agency and age in general. This is consistent with the theory, which states that Self-Care Agency gains a degree of perfection in adulthood but declines with advancing age. In this respect advancing age should be better interpreted as relatively far advancing. Relationship of Self-Care Agency and age was stronger for subjects without patient status than for all study subjects together. These findings again are supportive of construct validity of A.S.A.-A.

Factor-analysis revealed two relative robust factors across the sample of the entire study population of the research program and the subsamples of predominantly older subjects with health problems and predominantly younger healthy subjects. Factor robustness across studies was analysed first and used as the main criterium before ultimately deciding to compare factor structure with the patterns of clusters of Self-Care Agency - Power Components. This seems necessary in the light of the discussion of construct validity testing based on factor analysis of the more recently published Questionnaire on Perception of Self Care Agency P.S.C.A.Q. (Hanson, Bickel, 1985) generated by Weaver (1987). The psychometric properties of Bickel and Hanson's P.S.C.A.Q. were examined using a LISREL confirmatory factor analysis approach. A convenience sample of 462 non-institutionalized adults in North Western Ohio U.S.A. was used. Findings indicated that the factor structure of P.S.C.A.Q. was significantly different than that described by Bickel. Weaver concluded that these findings call construct validity of the P.S.C.A.Q. into question. This also makes clear that factor analysis is a somewhat controversial multivariate procedure. It involves a higher degree of subjectivity than one ordinarily finds in a statistical technique. It nevertheless is considered a highely powerfull and widely applied procedure and therefore needs attention (Polit, Hungler, 1987).

Orem cited in Hanson and Bickel (1985) has suggested three configurations of theoretical associations among Power Components of Self-Care Agency. Power components I - II - III form configuration A, IV, VI and VII form configuration $B$ and $\mathrm{IX}$ and $\mathrm{X}$ configuration $\mathrm{C}$. In all three configurations power components V 'motivation' and VIII 'skills' may be represented linked to the other Power Components. Comparison of results of this study with this theoretically suggested configuration leads to the following conclusions. Power components V and VIII are represented in all three theoretical configurations and as such may be expected to fluctuate in empirical findings. This indeed was the case in the findings of this study. Power components IX and $X$ could not empirically be identified as a stable third factor. They also did not consistently 
cluster to either factor one or factor two. This indicates that 'Integration and Priority setting' may be difficult to sort out as a distinct entity when measured across culturally different populations. Configuration $\mathrm{A}$ seems to reflect the general notion of 'energetic action' and as such it is comparable with factor 2 in this study. Configuration B seems to reflect the general notion of 'deliberate' of Self-Care Agency and as such is comparable to factor 1. Power Components do empirically cluster somewhat different within these configurations than theoretically suggested. Important to realise in this respect is the following. Power Components were considered by N.D.C.G. (1979) as formulations in need of review and testing. During scale development it was concluded that classification of the Power Components does not consist of mutually exclusive categories. Content analysis of the A.S.A.-A scale further revealed that many items reflect more than one Power Component. Within the context of this relative uncertainty similarity between general notions of theoretically suggested configurations of Power Components and extracted factors is considered as sufficient evidence for construct validity.

In addition to construct validity two measurement conditions were tested. $A$ significant positive correlation between Total A.S.A.A scores and Total Social Desirability scores would be indicative for response set bias. Pearson's $r$ was positive but not significant (H.22). Female patients had a significant higher score on Social Desirability. Pearson's correlation between A.S.A.-A and Social Desirability calculated for only female subjects was stronger but not significant. It can be concluded that the A.S.A.-A measured with a Dutch population is not significantly influenced by social desirability. Results also indicate a slight tendency that A.S.A.-A measurement with female subjects may produce some social desirable response set bias. A.S.A.-A was also tested for possible measurement error produced by variation in scale administration. No significant difference was found between mean Total A.S.A.-A score of patients to whom the scale was send by mail and the mean score of comparable patients to whom the scale was personally administered. Important in this respect is to note that study subjects were already acquainted with the scale from former measurements. It can be concluded that measurement error is absent at least for second or consecutive measurements, when A.S.A.-A is administered by mail.

In conclusion. Differences in operability of Self-Care Agency between groups of elderly in various degrees of dependency on nursing care could accurately be demonstrated in the predicted direction using the A.S.A.-A scale. Measurement of operability of Self-Care Agency with A.S.A.-A converged with measurement results on Kearney and Fleischer's 16 item version of the Exercise of Self-Care Agency scale. Measurement of operability of Self-Care Agency with A.S.A.-A also converged with response to the question 'How do you assess your capability to care for yourself with answering possibilities 
ranging from very bad to very good. Correlation was positive and significant. Both self-assessment of Total Activities of Daily living as well as assessment of A.D.L. by family and nurses were not related to Self-Care Agency as measured with Total A.S.A.-A. The A.S.A.-A scale could be used to empirically discriminate the concept of Self-Care Agency from the A.D.L.-concept with chronic reumatoid arthritic patients and older patients recently discharged from an acute care hospital. The relationship between Self-Care Agency and Self-Care as proposed in Orem's theory of Self-Care Deficit could be demonstrated with the A.S.A.-A scale both in situations of good and bad health. Positive change in Self-Care Agency as an effect of change in health related to coronary artery bypass surgery could be demonstrated using the A.S.A.-A scale: Consistent with the theory a significant positive relation between social support and Self-Care Agency of chronic reumatoid arthritic patients living at home could also be demonstrated with the A.S.A.-A scale. The more attentive, wellbalanced, self-confident and feminine college students qualified themselves the higher their operability of Self-Care Agency as measured with A.S.A.-A. Operability of Self-Care Agency was negatively correlated with age for persons of 65 years or older. A curvilinear pattern could be demonstrated between Self-Care Agency measured with A.S.A.-A and age in general. This again was consistent with the theory. Factor analysis revealed two relative robust factors. Factor one contained consistently Self-Care Agency - Power Components I Attention, IV Reasoning, and VII Knowledge and as such reflects the notion 'Deliberate'. Factor two contained consistently Self-Care Agency - Power Components II Energy and VI Decision making and as such reflects the notion of 'Action'. Similarity between these general notions of theoretically suggested configurations of Self-Care Agency - Power Components and the extracted factors was considered sufficient. In combination the above reported results mean strong evidence for construct validity of the A.S.A.-A scale. In addition it can be concluded that the A.S.A.-A scale used with a Dutch population is not significantly influenced by social desirability tendency. A.S.A.-A can be administered by mail without significant measurement error at least for second or consecutive measurements.

\subsection{Operational Consequences}

Based on conclusions in relation to reliability and validity in the first part of this chapter the following operational consequences can now be formulated for the Dutch version of the Appraisal of Self-Care Agency A.S.A.-A scale. Total number of scale-items will be reduced to 20 . Item numbers 7,12 and 24 will be dropped to improve internal consistency. Item number 4 will be dropped for reasons of parsimony. The new version of the A.S.A.-A scale will contain 8 negative and 12 positive items. Individual itemscore will be changed into Totally Disagree $=0$, Disagree $=1$, Neither Disagree Nor Agree $=2$, Agree $=$ 3 and Totally $\mathrm{Agree}=4$. After reversing scores of negative items the theoretical 
range for Total A.S.A.-A scores will be from 0 till 80 . The latter score indicates most operable Self-Care Agency. The scale bas a two factor structure. Factor one is called the 'Deliberate' Component of Self-Care Agency. Factor two is called the 'Action'. component of Self-Care Agency. When administered to older subjects with health problems Factor 1 consists of items $1,2,3,8,14,15$, $16,17,19$ and the items $5,10,18$ and 21. Factor 2 consists of items $6,9,11,13$ and 20 . When administered to young adult subjects without health problems the items $5,10,18$ and 21 change from Factor 1 to Factor 2. Table 6.1 summarizes these operational consequences. A new adapted version of the A.S.A.-A is presented. The new item order, the related old version item number, item nature, item content, factor structure and new scoring system are respectively given.

Table 6.1. New adapted version of $A_{\text {. }} S . A_{*}-A$ scale

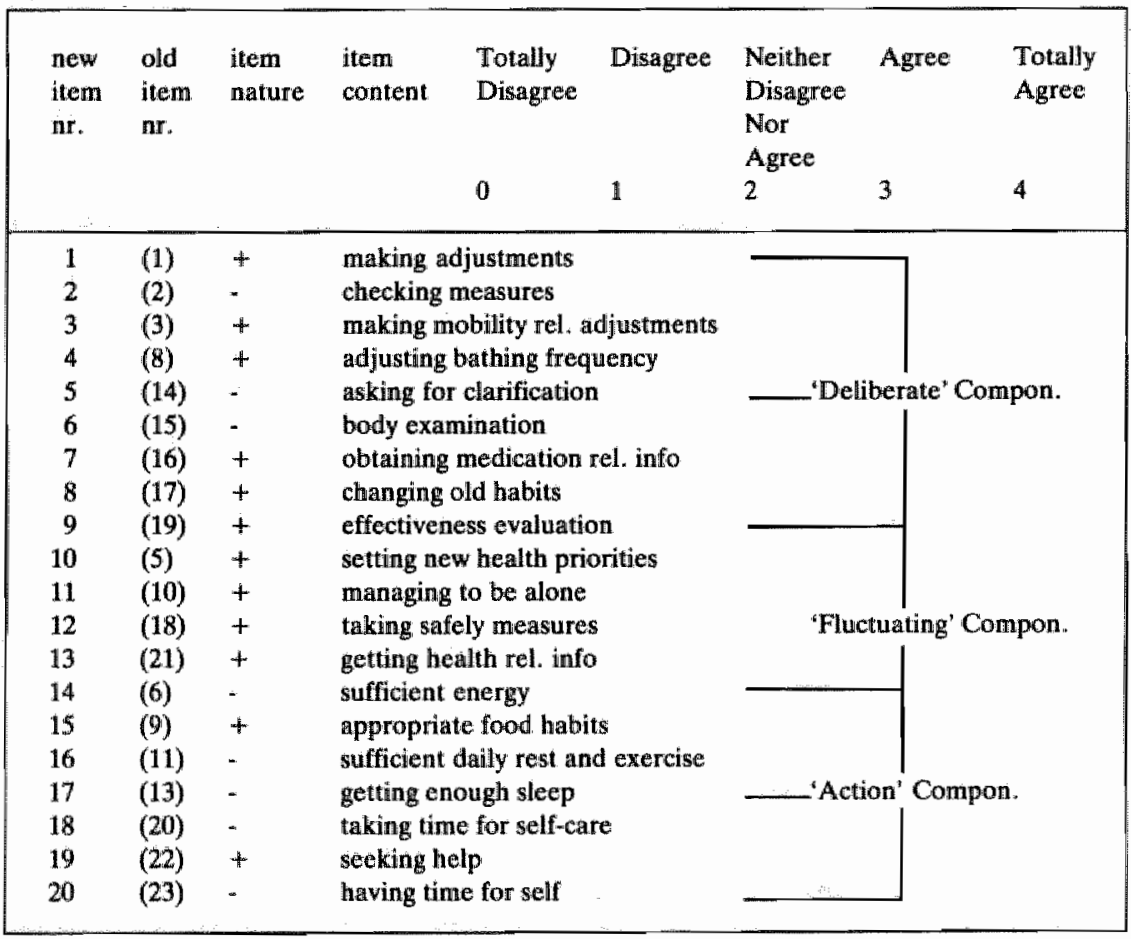

\subsection{Limitations and Implications}

The purpose of this study was reliability and validity testing of the A.S.A.-A scale. No systematic attempt has been made in this study to analyse construct 
validity or different reliability aspects of the appraisal of another A.S.A.-B scale. Additional analyses and studies are needed. As a consequence results obtained from reliability and validity testing of the A.S.A.-A scale may not be generalized to A.S.A.-B without additional testing. The purpose of this study was instrument testing. No attempt was made to study distribution of operability of Self-Care Agency within Dutch society in general or particular subcultures, age groups or patient categories. Nor was the purpose to systematically study predictors of Self-Care Agency. As such findings generated in this study which are related to these topics should be used only with reserve. In this study an instrument was tested which was developed to measure operability of Self-Care Agency as defined by the nurse-theorist D.E. Orem. As such results are restricted to operability and do not include adequacy or development of Self-Care Agency. The instrument measures Self-Care Agency within Orem"s theory and as such may not be a valid measurement instrument for other e.g. political, societal, sociological, psychological or idiosyncratic defined concepts refered to as 'Self-Care', 'Self-help' 'Zelfzorg', 'Zelfhulp'.

A major limitation in relation to designs used in the research program was the lack of a classic experimental design. Study VI: CABS was the only study of which the design is comparable to a quasi-experimental one. Due to practical reasons all other studies were correlational in nature. As a consequence interpretation of the nature of tested relationships is restricted. Not in all seven studies the same complete standardized set of background variables was measured. This was mainly related to logistical problems of coordinating a program of research of seven separate studies. Some compromising and creativity was needed to realize such a program with limited resources. It certainly has produced some restriction in the analysis of results. As far as total sample characteristics is concerned females were somewhat overrepresented with $60 \%$. Most study subjects lived in the province of Limburg which is located in the southern part of The Netherlands. The cultural orientation in the southern part of The Netherlands originally was characterized by the predominantly Roman Catholic religion of its population. In the study population relatively few subjects were represented in the age group of 25-45. Morbidity pattern of study subjects was in no way representative for the Dutch population in general. Furthermore no patients with known psychiatric disorders or mentally retarded persons were included in the study. Validity and reliability in use of the A.S.A.-A scalle with these populations needs to be established.

A major implication of this study for nursing theory is that results demonstrate that Orem's Self-Care Deficit theory is testable within Dutch culture. Results support propositions nr. 1 and $\mathrm{nr} .2$ of the theory of Self-Care Deficit and are congruent with presupposition nr. 2.1 of this theory. Results also support proposition nr. 5 of the theory of Nursing Systems. Results have generated new insights and questions which might refine Orem's theories. Research data can 
clearly be related to the phenomena of concern of the discipline of nursing. Orem's theory has structured and focused the thinking of this study. As such it has the potential to attract far more nurse-researchers and more enduring groups of adherents. They are needed to produce sufficient scientific empirical support. Marx (1963) statement should keep our endeavours focused 'The most ingenious theories are of limited value untill empirical tests are produced'.

A major implication for nursing research is that a tested, reliable and valid instrument is available to measure operability of Self-Care Agency. This can be considered an important variable of concern in nursing and health care research. Systematic study is needed of distribution or 'prevalence' of Self-Care Agency within society in general and subpopulations in order to develop age, sex, morbidity and culture specific referent values. Systematic study is needed to explore strong and manipulable Sell-Care Agency predictors or determinants. Experiments are needed to systematically evaluate interventions aimed to increase operability of Self-Care Agency.

A major implication for nursing practice is the availability of an instrument to classify patients into groups of low, medium, and high A.S.A.-A scorers. The study with coronary bypass surgery patients has produced a clear indication for the practical value of such a classification. Low A.S.A.-A scorers were slow and unpredictable post-operative Self-Care Agency improvers. They certainly need more and earlier extra attention and Supportive Educative Nursing Care. The study further produced evidence that operability of Self-Care Agency does indeed improve after Coronary Bypass Surgery. It seems practical to generalize this finding to more comparable nursing situations. This study also produced evidence that Self-Care Agency and Social Support are significantly positively related. An important implication for nursing practice could be more systematic attention for and inclusion of patients' supporters in nursing care. In too many situations in nursing practice patients still are 'stripped' from their cultural and social context. A last important implication of this study for nursing practice is that results demonstrate that Self-Care Agency empirically can be discriminated from Activities of Daily Living A.D.L. A.D.L. in its instrumental use seems to focus attention specifically on a few functions of patients which cannot be performed anymore. More systematic paying attention to patients' Self-Care Agency in nursing practice may focus both nurse and patient more on the latter's potential to develop. This study's results indicate that that still is possible at (far) advancing age. 


\section{SUMMARY}

The purpose of this study is reliability and validity testing of the Dutch version of the Appraisal of Self-Care Agency A.S.A.-scale. Self-Care Agency is a major concept in the Self-Care Deficit theory of the North American nurse Dorothea Orem (Orem, 1971, 1980, 1985). It refers to a person's acquired ability for deliberate action in order to meet one's continuing requirements for care. More specifically the kind of care that maintains life, maintains and promotes health, development and general well-being. This ability can vary in terms of development, operability and adequacy. As such this concept is different from Self-Help or Independence in Activities of Daily Living, A.D.L.

The A.S.A.-scale was developed as a self-appraisal instrument (form A) for use with adult populations in various states of health. An appraisal of another form B also exists which can be completed by a nurse or a significant other. Conceptually the instrument measures operability of Self-Care Agency. A Likert type of scaling is used with five response categories. A total of 24 items both positively and negatively stated were included in the A.S.A.-A scale version tested in this study. Scores of negative items were reversed and individual items were combined into an unweighted summative scale. Content validity testing has been done in the U.S.A. by a panel of nurse experts and Dorothea Orem.

Based on measurement theory and Orem's Self-Care Deficit theory twenty three hypotheses were formulated for reliability and validity testing. In terms of reliability internal consistency, equivalence and stability were addressed. $\mathrm{Hy}$ potheses were stated in relation to Cronbach's Alpha $(\geq .70)$, item to total correlations ( $\geq .30$ ). Item discrimination between high and low Total scores, percentage of explained variance and item loadings on the first component of the unrotated factor analysis matrix $(\geq .35)$. Moderate positive correlations were hypothesized between subjects Total A.S.A.-A scores and Total A.S.A.-B scores given by nurses or significant others. For a six weeks and six months time interval Total A.S.A.-A were expected to correlate strongly and moderately in a positive direction. Validity testing was limited to construct validity due to the theoretical nature of the Self-Care Agency concept. This was done by means of the Known Groups Technique, by demonstration of Convergence and Discriminability, by examination of relations within Orem's theory and Factor-analysis. Hypotheses were formulated to test Self-Care Agency 
measured with the A.S.A.-A scale in relation to dependency of institutionalized (nursing) care, to a one sentence statement about Self-Care capability, to A.D.L. measurements, to Self-Care, to change in Health State, to Social Support, Sex-Stereotyped Self-Image and Age. Two last hypotheses dealt with possible contribution to measurement error of Social Desirability and variation in scale administration.

Hypotheses were tested in a program of seven Research Studies. The first study was designed to measure difference in Self-Care Agency between elderly living independently in the community, service-flat residents, personalized care facility residents and nursing home patients. Short time stability testing was also included in the design. The second study investigated the relationship of SelfCare Agency. A.D.L. and Social Support of Reumatoid Arthritic patients at home. A.D.L. was measured with Katz' A.D.L.-index (Katz e.a., 1963) and Social Support with the Norbeck Social Support Questionnaire (Norbeck e.a., 1981). Study three was designed to compare Self-Care Agency of institutionalized rehabilitation patients measured by A.S.A.-A and a shortened 16 item version of Kearney and Fleischer's E.S.C.A.-scale (Kearney and Fleischer, 1979). In the fourth study acute care hospital patients' Self-Care Agency appraised by themselves, by nurses and significant others was compared. The fifth study explored Self-Care Agency and A.D.L. of patients discharged from an acute care hospital. In this study A.D.L. was measured with the Physical A.D.L. component of Pfeiffer's Multidimensional Functional Assessment Questionnaire (Pfeiffer, 1975). Study six was designed as a longitudinal study to investigate change in patient's Self-Care Agency in relation to Self-Care after coronary bypass surgery. Self-Care was measured with a revised version of Eisler, Wolfer and Diers Patient Recovery Inventory (Eisler e.a., 1972). In this study variation in scale administration was also tested as well as the influence of Sociall Desirabiliy measured with an adapted version of Crowne and Marlowe's SD scale (Crowne and Marlowe, 1964; Visser, 1984). In the last study Self-Care Agency of healthy college students was compared with their sex-stereotyped Self-Image, measured with the Groningen Androgynie Schaal (de Graaf, 1984) an adapted Dutch version of Bem Sex Role Inventory (Bem, 1974). All designs used were correlational with the exception of study design six. Sample selection and characteristics as well as instrumentation and procedure of data-collection of the seven studies were described separately.

Combined data of the seven studies included A.S.A.-A scores of 676 subjects. They completed a total of 908 A.S.A.-A measurements. The majority of the subjects were female. Mean age was 52 years with a range from 18 till 97 . At the time of the first measurement 383 subjects lived at home. The others stayed in health care facilities. The majority of the subjects had patient status. Most frequent medical diagnoses were diseases of the circulatory system and diseases of the musculo-sceletal system. Subjects mean years of education was 10.97 years. Theoretically Total A.S.A.-A scores could range from 24 till 120. For the entire population of the research program the empirical range was 37 till 117 . 
Mean score was 89.6. Shape of score distribution was unimodal and negatively skewed. Response frequency of item number 4 was extremely skewed consistently across all studies.

Cronbach's Alpha calculated for the entire study population's first measurement was .77. All separately calculated alpha"s were higher than 70 with the exception of two borderline cases of .69 . In combination results for other hypotheses formulated in relation to internal consistency were supportive for unidimensionality and demonstrated that homogeneity of the scale can be increased by dropping items nr. 7,21 and 24 . Results for hypotheses formulated in relation to equivalence demonstrated that self-appraisal scores cannot be considered identical to appraisal of another scores. In general Total A.S.A.-A scores correlated positively with A.S.A.-B scores and could be considered equivalent measures. Results suggested that this may not hold under conditions of important negative or positive change in Self-Care Agency or conditions of less or lack of human interaction and communication. Hypotheses testing stability for six weeks and six months time-interval were strongly supported.

In terms of construct validity difference in mean A.S.A.-A score between groups of elderly was significant with the exception of the difference between personalized care facility residents and nursing home patients. Nevertheless the difference was in the expected direction. Correlation between A.S.A.-A scores and scores on the 16 item version of the E.S.C.A.-scale was significantly moderate. With only one exeption correlations between A.S.A.-A and the one sentence statement about Self-Care Capability were significantly positive across the studies. Both self-assessment of Total A.D.L. as well as A.D.L. assessment by nurses and family members were not significantly correlated to Total A.S.A.-A scores. The proposed positive relationship of Self-Care Agency and Self-Care could be demonstrated in situations of poor and improved health state. Coronary artery bypass patient's pre-operative mean Total A.S.A.-A score was significantly lower than their six and twelve months postoperative mean score, whille no significant difference was found between mean six and twelve months scores. A significant positive relation was demonstrated between Social Support and Self-Care Agency. Sex-stereotyped Self-Image correlated significantly with college students' Self-Care Agency. The more attentive, well-balanced, self-confident, self-assured and feminine students qualified themselves the higher their Total A.S.A.-A scores. Operability of Self-Care Agency was negatively correlated with age for persons of 65 years or older. Relationship was stronger for subjects without patient-status than for all subjects together. Factor-analysis revealed two relatively robust factors across subsamples. Factor one was labeled 'Deliberate' and factor two as 'Energetic Action'. Similarity between the theoretical structure underlying Self-Care Agency and extracted factors was considered sufficient to support construct validity. No significant positive correlation was demonstrated between Total A.S.A.-A and Total Social Desirability scores. No significant difference was found between mean total A.S.A.-A score of subjects to whom the scale was 
mailed and of comparable subjects to whom the scale was personally administered.

It was concluded that the A.S.A.-A scalle can be used in an acceptable way as a reliable, internal consistent instrument for group level comparisons of adult healthy subjects, patients at home and in health care institutions even under extreme measurement conditions. The scale should be used with caution for decisions about an individual. It was also concluded that the above reported results in combination meant strong evidence for construct validity of the A.S.A.-A scale. The following scale changes were suggested. To reduce total item numbers to 20 . To improve internal consistency item numbers 7,12 and 24 can be dropped. For reasons of parsimony item number 4 can be deleted. Implications for nursing theory, research and practice conclude this report on reliability and validity testing of the A.S.A.-scale. 


\section{SAMENVATTING}

Het doel van deze studie is de betrouwbaarheid en validiteit te testen van de Nederlandse versie van de A.S.A.-schaall ter beoordeling van Zelfzorg-Vermogen. Zelfzorg-Vermogen is een belangrijk begrip uit de theorie over gebrek aan Zelfzorg van de Noord-Amerikaanse verpleegkundige Dorothea Orem (Orem, 1971, 1980, 1985). Zelfzorg-Vermogen slaat op het aangeleerde vermogen van iemand om weloverwogen te handelen bij het voldoen aan eigen steeds terugkerende behoeften aan zorg. Daarbij gaat het om de zorg om in leven te blijven, om je gezondheid te behouden en te bevorderen en om je ontwikkeling en een algemeen gevoel van welbevinden te bevorderen. Dit vermogen kan variëren in mate van ontwikkeling, operationaliteit en adequaatheid. Zelfzorg-Vermogen is dus een ander begrip dan Zelfhulp of Zelfredzaamheid bij Activiteiten van het Dagelijkse Leven, A.D.L.

De A.S.A.-schaal is ontwikkeld als een zelf-beoordelings instrument (versie A) om te gebruiken bij volwassenen in verschillende omstandigheden van ziekte en gezondheid. Er bestaat ook een zogenaamde B versie van de schaal, waarmee men een ander kan beoordelen. Dit kan bijvoorbeeld worden gedaan door een verpleegkundige of een zogenaamde significante andere, d.w.z. een partner, kind, goede vriend of vriendin. Begripsmatig wordt het operationeel zijn van Zelfzorg-Vermogen gemeten met de A.S.A.-schaal. Het is een Likert type schaal met 5 antwoord mogelijkheden. In totaal bestaat de A.S.A.-schaal, die in deze studie is getest uit 24 uitspraken. Sommige daarvan zijn positief andere negatief gesteld. De scores van de negatieve items worden omgedraaid. De scores van de afzonderlijke items worden opgeteld tot een ongewogen totaalscore. De schaal is in de U.S.A. op inhoudsvaliditeit getest door een panel van verpleegkundige experts en door Dorothea Orem zelf.

Op basis van algemene meet-theorie en Orem's theorie over gebrek aan Zelfzorg zijn drieentwintig hypothesen geformuleerd over de betrouwbaarheid en validiteit van de schaal. Wat betreft betrouwbaarheid komen de aspecten interne consistentie, equivalentie en stabiliteit aan de orde. Specifieke hypotheses werden geformuleerd ten aanzien van Cronbach's Alpha ( $\geq .70$ ), correlaties van itemscores met totaal scores $(\geq .30)$, het discriminerend vermogen van items tussen hoge en lage Totaalscores, het percentage verklaarde variantie van en de lading van items op de eerste component van de ongeroteerde factor-analyse matrix ( $\geq .35$ ). Matig positieve correlaties werden verondersteld 
tussen Totale A.S.A.-A scores van onderzochte personen en hun Totale A.S.A.-B scores gegeven door verplegenden en significante anderen. Een sterk positief werband werd verwacht voor opeenvolgende A.S.A.-A scores met een tussenliggende periode wan 6 weken en een matig positief verband voor een tussenliggende periode van 6 maanden. Vanwege het abstracte karakter van het begrip Zelfzorg-Vermogen is het testen van validiteit beperkt tot begripsvalidering. Dit is gedaan door middel van de methode van Bekende Groepen, door het aantonen van convergentie en divergentie, door het nagaan van relaties binnen Orem's theorie en door factor-analyse. Hypothesen werden geformuleerd woor de relatie van Zelfzorg-Vermogen zoals gemeten wia de A.S.A.-A schaal met het verschil in afhankelijkheid van geïnstitutionaliseerde (verpleegkundige) zorg bij ouderen, met een éénregelige uitspraak over de mogelijkheid om voor zichzelf te zorgen, met A.D.L.-metingen, met Zelfzorg, met verandering in gezondheidstoestand, met sociale ondersteuning, sexe getypeerd zelfbeeld en leeftijd. Twee hypothesen gingen tot slot over de mogelijke invloed van sociale wenselijkheid en het variëren van afname condities van de meetschaal op meetfouten.

Deze hypothesen zijn getoetst in een onderzoeksprogramma bestaande uit zeven afzonderlijke studies. De eerste studie was opgezet om het verschil te testen in Zelfzorg-Vermogen tussen ouderen die zelfstandig thuis wonen, ouderen in een service-flat, in een verzorgingstehuis en een verpleeghuis. In de onderzoeksopzet was eveneens opgenomen het testen van stabiliteit over een 6 weken durende periode. De tweede studie onderzocht de relatie tussen Zelfzorg-Vermogen, A.D.L. en Sociale Ondersteuning bij thuis verblijvende reumatolde arthritis patiënten. A.D.L. werd gemeten met behullp van de A.D.L.index (Katz e.a., 1963) en Sociale Ondersteuning met de N.S.S.Q.-vragenlijst (Norbeck, 1981). De derde studie was opgezet om bij opgenomen revalidatie patiënten het Zelfzorg-Vermogen te meten zowel met de A.S.A.-A schaal als met een verkorte versie van de E.S.C.A.-schaal (Kearney en Fleischer, 1979). De vierde studie betrof een vergelijking tussen de beoordeling van ZelfzorgVermogen van patiënten opgenomen in een Algemeen Ziekenhuis door de patienten zelf, door verpleegkundigen en door significante anderen. In de vijfde studie is nagegaan in hoeverre Zelfzorg-Vermogen en A.D.L. samenhangen bij patiënten die recent ontslagen zijn uit een Algemeen Ziekenhuis. A.D.L. is in dit onderzoek gemeten met de Fysieke A.D.L. component van de M.F.A. Q.-vragenlijst (Pfeiffer, 1975). Studie zes was opgezet als een longitudinale studie. Nagegaan is de verandering in Zelfzorg-Vermogen in relatie tot Zelfzorg bij patiënten vóor en na een coronair bypass operatie. Zelfzorg is gemeten met een gewijzigde versie van de P.R.I.-vragenlijst van Eisler, Wolfer en Diers (1972). In deze studie is ook nagegaan welke invloed variatie in de wijze van afname van de vragenlijst en sociale wenselijkheids-tendenties bij respondenten heeft op Totaal Scores. Sociale wenselijkheid is gemeten met een aangepaste versie (Visser, 1984) van Crowne and Marlowe's SD Schaal (1963). In de laatste studie is nagegaan in hoeverre Zelfzorg-Vermogen een samenhang 
vertoont met sexe-getypeerd Zelfbeeld bij gezonde H.B.O.-studenten. Sexegetypeerd Zelfbeell is gemeten met de Groningen Androgynie Schaal (de Graaf, 1984), een in Nederland ontwikkelde schaal op basis van de S.R.I.schaal van Bem (1974). Alle studies zijn als zuiver correlationele onderzoeken opgezet met uitzondering van studie zes.. De selectie van proefpersonen, hun kenmerken, de gehanteerde instrumenten en de wijze van data-verzameling zijn afzonderlijk beschreven voor de zeven studies.

De gecombineerdle gegevens van de zeven studies omvatten A.S.A.-A scores van 676 proefpersonen. In totaal hebben deze proefpersonen 908 A.S.A.-A scores geproduceerd. De meerderheid van de proefpersonen bestond uit vrouwen. De gemiddelde leeftijd was 52 jaar met een jongste deelnemer van 18 en een oudste van 97 . Ten tijde van de eerste meting verbleven 383 personen thuis. De overigen verbleven in instellingen voor gezondheidszorg. De proefpersonen waren in meerderheid patiënten. De meest voorkomende medische diagnoses waren ziekten vam hart en bloedvaten en zieken van het spier-en beenderengestel. Gemiddeld hadden de proefpersonen 10.97 jaar schoolopleiding gevolgd. De theoretische score van de A.S.A.-A schaal kan variêren van 24 tot 120. Voor de totale onderzoekspopulatie liep de empirische range van 37 tot 117. De gemiddelde score bedroeg 89.6. De frequentie verdeling van de scores was unimodaal en scheef negatief verdeeld. Consistent over alle studies vertoonde alleen item nummer 4 de extreem scheve antwoord frequentie van 90 tot $100 \%$ geheel mee eens.

De Cronbach's Alpha berekend voor de eerste meting bij de gehele onderzoekspopulatie bedroeg .77. Alle afzonderlijk berekende alpha's waren hoger dan .70 met uitzondering van twee grensgevallen van .69. De gecombineerde resultaten voor de andere hypotheses, opgesteld met betrekking tot interne consistentie, betekenden steun voor een unidimensioneel karakter van de schaal. $\mathrm{Zij}$ toonden voorts aan dat de homogeniteit van de schaal vergroot kan worden door verwijdering van de items nummer 7,21 en 24 . De resultaten voor de hypothesen over equivalentie toonden aan dat zelf-beoordelings scores niet als identiek beschouwd kunnen worden an scores van een beoordeling door anderen. In het algemeen was er een positief verband tussen A.S.A.A en A.S.A.-B scores en konden beide metingen als equivalenten beschouwd worden. De resultaten suggeren tevens dat dit wellicht niet opgaat bij grote positieve of negatieve veranderingen in Zelfzorg-Vermogen of bij geringe of gebrek aan menselijke interactie en communicatie. De hypotheses over stabiliteit voor een zes weken en zes maanden durende periode werden duidelijk bevestigd.

Wat begripsvaliditeit betreft werden de volgende resultaten gevonden. Het verschil in gemiddelde A.S.A.-A score tussen de diverse groepen ouderen was significant met uitzondering van het verschil tussen verzorgingstehuis bewoners en verpleeghuis patiënten. Niettemin lag het verschil in de richting zoals verwacht. De correlatie tussen A.S.A.-A scores en de scores op de verkorte versie van de E.S.C.A.-schaal was significant en matig positief. Op een na waren alle berekende correlaties tussen A.S.A.-A en een éénregelige uitspraak over de 
mogelijkheid om voor jezelf te zorgen significant positief. Zowel de zelfbeoordeling van A.D.L. als de A.D.L.-beoordeling door verpleegkundigen en familieleden hing niet significant samen met Totale A.S.A.-A scores. De vooronderstelde positieve relatie tussen Zelfzorg-Vermogen en Zelfzorg kon worden aangetoond zowel in situaties met een minder goede als met een verbeterde gezondheidstoestand. De gemiddelde pre-operatieve score op de A.S.A.-A van coronair bypass patiënten was significant lager dan hun 6 en 12 maanden post-operative gemiddelde score. Geen significant verschil kon worden aangetoond tussen de score van deze patiënten op 6 en 12 maanden na de operatie. Een significant positief verband kon worden aangetoond tussen Sociale Ondersteuning en Zelfzorg-Vermogen. Het sexe-getypeerd zelfbeeld van H.B.O.-studenten hing significant samen met hun Zelfzorg-Vermogen. Hoe meer attent, evenwichtig, zelfbewust, zelfverzekerd, vastberaden en vrouwelijk de studenten zichzelf kwalificeerden deste hoger waren hun Totale A.S.A.-A scores. Het operationeel zijn van Zelfzorg-Vermogen hing bij personen van 65 jaar en ouder negatief samen met leeftijd. Deze relatie was sterker voor niet-patiënten dan voor alle proefpersonen tesamen. Factoranalyse toonde het bestaan van twee tamelijk robuste factoren aan. Deze bleven constant bij anallyse van sub-populaties. De eerste factor kreeg het labell 'Weloverwogen' en de tweede factor het label 'Energieke Actie'. De overeenkomst tussen de theoretische structuur die aan het begrip Zelfzorg-Vermogen ten grondslag ligt en de beide factoren vormt een laatste ondersteuning voor de begripsvaliditeit van de A.S.A.-schaal. Tussen sociale wenselijkheidsscores en A.S.A.-A scores werd geen significante correlatie aangetoond. Evenmin werd een significant verschil gevonden tussen de gemiddelde A.S.A.-A score van een groep proefpersonen aan wie de schaal per post was toegestuurd en een vergelijkbare groep proefpersonen aan wie de schaal persoonlijk was voorgelegd.

De volgende conclusies konden worden geformuleerd. Het gebruik van de A.S.A.-A schaal als een betrouwbaar, intern consistent instrument is acceptabel voor groepsvergelijking van gezonde proefpersonen, vam patiënten thuis en in instellingen voor gezondheidszorg zelfs onder extreme meet-omstandigheden. De schaal dient met terughoudendheid te worden gehanteerd bij beslissingen over een individu. In combinatie betekenen bovenvermelde resultaten sterke bewijsvoering voor begripswaliditeit van de A.S.A.-A schaal. De volgende veranderingen in de schaal worden voorgesteld. Reductie tot een schaall met 20 items. Ter vergroting van de interne consistentie kunnen de items nummer 7, 12 en 24 worden verwijderd. Uit een oogpunt van overbodigheid kan item nummer 4 weggelaten worden. Het verslag van dit onderzoek naar de betrouwbaarheid en validiteit van de A.S.A.-A schaal eindigt met enkele slotopmerkingen over consequenties voor verplegingswetenschappelijke theorievorming en onderzoek en voor de verpleegkundige praktijk. 


\section{REFERENCES}

Achterberg, M. van (1988). Sexe en Zelfzargvermogen. Unpublished Master Thesis Unwversity of Limburg, Maastricht.

American Nurses' Association (1980). Nursing : A Social Policy Snatement. Kansas City.

Backscheider, J. (1974). Self-Care Requirements, Self-Care Capabilities and Nursing Systems in the Diabetic Nurse Management Clinic. American Journal of Public Health, 64, 1138-1146.

Bem, S.L. (1974). The measurement of Psychological Androgyny. Jounal of Consuling and Clinical Psychology, 42, 155-162.

Bem, S.L., Martyna, W., Watson, C. (1976). Sex Typing and Androgyny : Further Exploration of the Expressive Domain. Journal of Personality and Social Psychology, 34, 1016-1023.

Bergner, M., Bobbitt, R.A., Kressel, S., Pollard, W.E., Gilson, B.S. Monis, J.R. (1976). "The Sickness Impact Profile : Conceptual Formulation and Methodology for the Development of a Health Status Measure. Intemational Journal of Heallh Services, 6, 393-415.

Boderie, J.M.H.V., Sijstermans, L.G.D.C. (1986). Professionele Zorg of Mantelzorg : een Kivestie van Opvatting. Unpublished Master Thesis University of Limburg, Maastricht.

Boshouwers, R. (1987). Professionele Zorg, Aanvulling op Niet-professionele Zorg en Zelfzorg? Unpublished Master Thesis University of Limburg, Maastricht.

Brink-Tjebbes, J. van den. (1979). Het Leergebied Verpleegkunde I, II, III, IV. Tijdschrift voor Ziekenverpleging, 32 (19), 881-884, (20), 913-917, (22), 1021-1027, (23), 1072-1077.

Brink-Tjebbes, J. van den. (1982). Methodiek en systematiek van het Verplegen I, II. Tijdschrift voor Ziekenverpleging, 35 (1), 6-8, (2), 53-56.

Brouns, G. \& Evers, G. (1982). Ondersteunen van Zelfzorg : het Wezenskenmerk van Verplegen? Tijdschrift woor Ziekenverpleging, 35 (13), 414-418, (14), 453-458.

Brugge, P.A. (1981). The Relationship between Family as a Social Support System, Health Status and Exercise of Self-Care Agency in the Adult with a Chronic Mhress. Dectoral Dissertation. Detroit : Wayne State University. (University Microfilms no. 82-09277).

Campbell, A., Converse, P., Rogers, W. (1976). The Quality of American Life. New York : Russell Sage Foundation.

Carmines, E.G., Zeller, A.R. (1979), Reliability and Validity Assesstuent. Beverly Hills : Sage.

Central Bureau of Statistics (1986). Plaatsmamenklapper : Namen vam Platisen met Geografische Gegevens. 's-Gravenhage : Staatsuitgeverij.

Central Bureau of Statistics, Ministry of Welfare, Health and Cultural Affairs (1986). Compendium Health Statistics of the Netherlands. "s-Gravenhage : Stalatsuitgeverij.

Courtens, A.M. (1987). De Bekwaamtheid van Verpleegkundigen inzake he! Inschatten wan het Zelfzorguermogen van Patienten. Unpublished Master Thesis University of Limburg, Maastricht.

Crohnbach, L.J. (1971). Test Validation. In: R.L. Thorndike (ed). Educational Measurement. Washington D.C. : American Council on Education.

Crowne, D. and Marlowe, D. (1964). The Approval Motive. New York: Wiley.

Denyes, M.J. (1980). Development of an Instmument to Measure Self-Care Agency in Adolescents. Doctoral Dissertation. Ann Arbor : University of Michigan. (University Microfilms no. 80-25672), 
Dodd, M.J. (1980). Enhancing Self-Care Behaviors through Infarmational Interventions in Patients with Cancer who are Receiving Chemotherapy. Doctoral Dissertation. Detroit : Wayne State University. (University Microfilms no. 81-17053).

Eisler, J., Wolfer, J.A., Diers; D. (1972). Relationship Between Need for Social Approwal and Postoperative Recovery and Welfare. Nursing Research, 22, 394-401.

Evans; L. K. (1979). The Relationship of Need A wareness, Locus of Control, Health State and Social Siupport System to Social Interaction as a Form of Self-Care Behavior Among Elderly Residents of Public Housing. Doctoral Dissertation. Washington D.C. : Catholic University of America. (University Microfilms no. 80-13414).

Ever, G. (ed.). (1981), Verpleegkundige Grondbegrippen. Lochem : De Tijdstroom.

Evers, G.C.M., Isenberg, M.A., (1987). Reliabillity and Validity of the Appraisal of Self-Care Agency A.S.A.scale. In: International Nursing Research Conference Abstracts. Kansas City: American Nurses Association.

Evers, O.C.M., Isenberg, M., Philipsen, H., Brouns, G., Hallfens, R., Smeets, H. (1986). The Appraisal of Seli-Care Agency A.S.A.-scale: Research Program to Test Reliability and Validity. In: Stinson, S.M., e.a. (cd.). Proceeding of the International Nursing Research Conference. Edmonton: Uniwersity of Alberta.

Fawcett, J. (1984). Analysis and Evaluation of Conceptual Models of Nursing. Philadelphia: F.A. Davis.

Fawceti, J. \& Downs, F.S. (1986). The Relationship of Theory and Research. Norwalk, CT : Appleton Century Crofts.

Fernster, J.J. (1983). A Comparison of Patient and Nurse Perceptions of Patients' Self-Care Deficits Associated with Cancer Chemotherapy. Doctoral Dissertation. Birmingham : University of Alabama. (University Microfilms no. 84-14550).

Gast, H.L. (1983). The Relationship between Stages of Ego Development and Developmertal Stages of Heallh Self-Care Operations, Doctoral Dissertation. Denton: Texas Women's University. (University Microfilms no. 84-01198).

Graaf, A. de (1984). Konstruktie van een Verbeterde Versie van de Groninger Androgynie Schaal (GRAS). Heymans Bulletins, Psychologische Instituten R.U. Groningen, HB-84-710-EX.

Guilford, J. (1954). Psychometric Methods. 2nd ed. New York: McGraw-Hill.

Gunter, L.M. (1962): Notes on a Theoretical Framework for Nursing Research. Nursing Research, $11,219-222$.

Hanson $_{i}$ B. \& Bickel, L. (1985). Development and Testing of the Questionnaire on Perception of Self-Care Agency. In J. Riehl-Sisca (ed.). The Science and Art of Self-Care. Norwalk, C.T. : Appleton-Century-Crofts.

Hanson, N.R. (1958). Patterns of Discovery. Cambridge : Cambridge University Press.

Hardy, M.E. (1978). Perspectives on Nursing Theory. Advances in Nursing Science, 1, 27-48.

Hattinga Verschure, J.C.M. (1977). Het Verschijnsel Zorg. Lochem: De Tijdstroom.

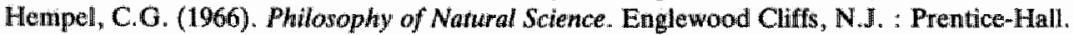

Henderson, V. \& Harmer, B. (1955). Textbook of the Principles and Proctice of Nursing (5th ed.). New York: Macmillan.

Hermans, H.J.M. (1967). Motivatie en: Prestatie. Dissertation Katholieke Universiteit Nijmegen. Amsterdam : Swets \& Zeitlinger.

Horn, B. \& Swain, M. (1977). Development of Criterion Measures of Nursing Care. Ann Arbor: University of Michigan.

Hungelman, J.A. (1984). Components of Self-Care Ability of Older Persons with Chronic Disease. Doctoral Dissertation. Chicago: Rush University. (University Microfilms no. 84-20074).

lsenberg, M.A (1986). Theoretical Analysis of the ESCA Scale as a Valid Measure of Exercise of Self-Care Agency. In Proceedings International Nursing Resiearch Canference. Edmonton: University of Alberta.

Isenberg, M.A., Evers, G.C.M., Phillipsen, H., Brouns, G. (1989). The Appraisal of Self-Care Agency (ASA) Scale : Development and Testing of a Measurement Instrument. Nursing Research (Submitted for publication). 
Johnson, D.E. (1980). The Behavioral System Modell for Nursing. In J.P. Riehl \& Roy, C. (eds.). Conceptual Modets for Nursing Practice (2nd ed.). New York: Appleton Century Crofts.

Johnson, D.E. (1961). The Significance of Nursing Care American Joumat of Nursing, 61, (11) 63-66.

Jong, J.H.J. de. (1983). Basiselementem woor de Verpleegkundige Theorie en Prakijik. Amsterdam : Elsevier.

Kahn, R.L. (1979). Aging and Social Support. In: M. W. Riley (ed.). Aging from Birth to Death; Interdisciplinary Perspectives. American Association for the Advancement of Science, Selected Symposium 30. Boulder, Colo. : Westwiew Press. 77-91.

Kalter, A.G. (1987). Betrouwbarheid en Validireil wan de ASA-schaal bij Meting van Zelfzorgwermogen wan Revalidanien. Unpublished Master Thesis University of Limburg, Maastricht.

Katz, S., Ford, A.B., Moskowitz, R.W., Jackson, B.A., Jaffe, M.W. (1963). Studies of Mlness in the Aged. The Index of ADL : A Standardized Measure of Biological and Psychosocial Function. Joumal of the American Medical Association, 185, 914-919.

Katz, S., Acpom, C.A. (1976). A Measure of Primary Sociobiological Functions. International Journal of Health Services, 6, 493-507.

Katz, S.M., Hedrick, S.C., Henderson, N.S. (1979). The Measurement of Long Term Care Needs and Impact. Health and Medical Care Services Review, 2, 3-20.

Katx, S. (1983). Assessing Self-Maintenance: Activities of Daily Living, Mobility and Instrumental Activities of Daily Living. Joumal of the American Geriatrics Society, 31, 721-727.

Kearney, B. \& Fleischer, B. (1979). Development of an Instrument to Measure Exercise of Self-Care Agency. Research in Nursing and Healih, 2, 25-34.

Kerlinger, F.N. (1973). Foundations of Behavioral Research. 2nd ed. New York : Holt, Rinehart and Winston Inc.

Kim, J., Mueller, C.W. (1982). Factar Analysis, Statistical Methods and Practical Issues. London: Sage.

King, I.M. (1971). Toward a Theory for Nursing : General Concepts of Human Behavior. New York : John Wiley.

King, I.M. (1981). A Theory for Nursing : Systems, Concepts, Process. New York: John Wiley.

Kotarbinski, T. (1966). Praxiology (Translated by O. Wojtasiewicz). New York: Pergamon.

Kuhn, T.S. (1970). The Structure of Scientific Revolutions (2nd ed.). Chicago: University of Chicago Press.

Leininger, M. (1978). Transcultural Nursing: Cancepts, Theories and Practices. New York : John Wiley.

Leininger, M. (1980). Caring : An Essential Hurnan Need. Thorofare, N.J. : C.B. Slack.

Leininger, M. (1984). Care: The Essence of Nursing and Health. Thorofare, N.J. : C.B. Slack.

Leininger, M. (1985). Transcultural Care Diversity and Universality : A Theory of Nursing. Nursing d Health Care, 6, 209-212.

Levin, L.S., Katz, A.M., Holst, E. (1976). Self-Care : Lay Initiatives in Health. New York: Prodist.

Levin, L.S. (1977). Self-Care and Health Planning. Social Policy, 8, 47.54.

Levine, M.E (1973), Introduction to Climical Nursing (and ed.). Philadelphia : F. A. Davis.

Maclver, J.P., Carmines, E.G. (1981). Umidimenstanal Scaling. Beverly Hills : Sage.

Macmurray, J. (1966). Self as Agent. London : Faber \& Faber.

Marx, M.H. (1963). Theories in Contemporary Psychology. New York : Macmillan.

Moos, R.H. (1974). The Social Climate Scales: An Overview. Palo Alto, Ca. : Consulting Psychologists Press.

Musci, E.C. (1983). Relationship between Family-Coping Strategies and Self-Care during Cancer Chemotherapy Treatments. Doctoral Dissertation. San Francisco: University of California. (University Microfilms no. 84-01307).

Nationale Raad voor de Volksgezondheid NRV (1984). Adwies Verpleegkundig Beroepsprofiel. Zoetermeer.

Nationale Raad voor de Volksgezondheid NRV (1988). Rapport Reacties Advies Verpleegkumdig Beroepsprofiel. Zoetermeer. 
Neves, E.P. (1980). The Relationship of Hospitalized Individuals' Cognitive Sinucture Regarding Health to Their Self-Care Behaviors. Doctoral Dissertation. Washington D.C. "Catholic University of America. (Uniwersity Microfilms no. 80-18514).

Neuman, B. (1982). The Neumam Systems Model: Application to Nursing Education and Practice. New York: Appleton Century Crofts.

Newman, M.A. (1972). Nursing's Theoretical Evolution. Nursing Outlook, 20, 449-453.

Newman, M.A. (1979). Theory Development in Nursing. Philadelphia: F.A. Davis.

Newman, M.A. (1983). The Continuing Revolution: A History of Nursing Sejence. In N.L. Chasca The Nursing Profession: A Time to Speek. New York: McGraw-Hill.

Newman, M. A. (1986). Healih as Expanding Consciousiness. St. Louis : Mosby.

Nightingale, F. (1859). Notes on Nursing : What it is, and What it is not. London : Harrison and Sons.

Norbeck, I.S., Linsey, A.M., Carnieri, V.L. (1981), The Development of an Instrument to Measure Social Support. Nursing Research, 30, 264:269.

Ninnully, J.C. (1978). Psychometric Theory. New York: MoGraw-Hill.

Nursing Development Conference Group N.D.C.G. (1973). Concept Formalization in Nursing : Process and Product (1st ed.). Boston : Little, Brown and Company.

Nursing Development Conference Group N.D.C.G. (1979). Concept Formalization in Nursing : Process and Product (2nd ed.). Boston : Little, Brown and Company.

Orem. D.E. (1956). Hospital Nursing Service : An Analysis. Indianapolis : Indiana State Board of Health.

Orem, D.E. (1959). Guides for Developing Curriculum for the Education of Practical Nurses. Washington: US Government Printing Office.

Orem, D.E. (1971). Nursing : Concepts of Practice (1st ed.) New York: McGraw-Hill.

Orem, D.E. (1980). Nursing: Concepts of Practice (2nd ed.) New York : McGraw-Hill.

Orem, D.E. (1985). Nursing : Concepts of Practice (3rd ed.). New York : McGraw-Hill.

Orlando, I.J. (1961). The Dynamic Nursie-Patient Relationship : Function, Process and Principles. New York: Putnam.

Parse, R.R. (1987). Nursing Science, Major Paradigms, Theories and Criniques. Philadelphia: W.B. Saunders.

Parsons, T. \& Shils, E. A. (ed.). (1951). Toward a General Theory of Action. New York : Harper \& Row.

Parsons, T. (1968). The Structure of Social Action. Vol. I. New York : Macmilan.

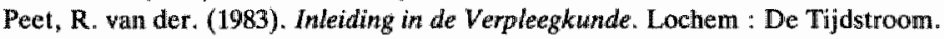

Peet, R. vas der. (1984). Verpleegkunde I. Lochem : De Tijdstroom.

Peet, R. van der. (1985). Verpleegkunde II. Lochem: De Tijdstroom.

Peplau, H. (1952). Interpersonal Relations in Nursing. New York: Putnam.

Pfeiffer, E. (ed.) (1975). Multidimensional Functional Assessment. Durham, North Carolinat : Duke University Center for the Study of Aging and Human Development.

Polìt, D.F., Hungler, B.P. (1987). Nursing Reseanch : Principles and Methods. 3rd ed. Philadelphia: J.B. Lippincott Co.

Remmen, J.W.M. (1985) Bejaardenoordbewoners of Verpleeghwispatienten. Dactoral Dissertation. Nijmegen : Catholic University.

Robinson, J.P., Shaver, P.R. (1975). Measures of Social Psychological Attitudes. Ann Arbor : Institute for Social Resenrch, The University of Michigan.

Rogers, M.E. (1970). An Introduction to the Theoretical Basis of Nursing. Philadelphia: F.A. Davis.

Roy, C. (1970). Adaptation: A Conceptual Framework for Nursing. Nursing Outlook, 18, 42-45.

Roy, C. (1984). Introduction to Nursing : An Adaptation Model (2nd ed.). Englewood Cliffs, N.J. : Prentice-Hall.

Roy , C. \& Roberts, S.L. (1981). Theory Construction in Nursing : An Adaptation Model. Englewood Cliffs, N.J. : Prentice-Hall.

Schiltmans, M.M.C. (1987). Het Thuisfunctioneren van Reumatoüde Arthritus Patienten. Unpu- 
blished Master Thesis University of Limburg, Masistricht.

Schrijnemaekers, V.J.J. (1986). Een Onderzoek naar de Betrouwbarheid en Vallitith wan de ASA-Beoordeling Zelf Forg-Vermogen Schaal. Unpublished Master Thesis University of Limburg, Maastricht.

Stultenbarger, N.E. (1984). A Q-Analysis of the Self-Care Abilines of Young, Schoolaged Children. Doctoral Dissertation. Birmingham : University of Alabama. (University Microfilins no. 84-26743).

Underwood, P.R. (1978). Nursing Care as a Determinant in the Dewelopment of Self-Care Behovior by Hospitalized Adult Schizophrenics. Doctoral Dissertation. San Francisco : University of Calffornia. (University Microfilms no. 79-18208).

Veen, H.G. van der (1979). Sekse Stereotypen. In: G.J. Kok et al. Vooroordeel en Discriminatie. Alphen aan den Rijn : Samsom.

Visser, A.J.P. (1984). De Beleving van het Verblijf in het Algemene Ziekenhuis. Dissertation VU Amsterdam. Assen : Van Gorcum.

W.H.O. (1975). International Statistical Classification of Diseases and Causes of Death. Ninth Revision. Geneva.

Wald, F.S. Leonard, R.C. (1964). Towards Development of Nursing Practice Theory. Nursing Research, 13, 309-313.

Walker, L.O. \& Avant, K.C. (1983). Strategies for Theory Construction in Nursing. Norwalk, CT : Appleton Century Crofts.

Walker, L.O. (1986). Theory, Practice and Research in Perspective. In L.H. Nicoll (ed.). Perm spectives on Nursing Theory. Boston : Little and Brown.

Weaver, M.T. (1987). Perceived Self-Care Agency : A LISREL Factor Analysis of Bickel and Hanson's Questionnaire. Nursing Research, 36, 381-387.

Westerlaak, J.M. van, Kropman, J.A., Collaris, J.W.M. (1975). Beroepentiklapper. Nijmegen : In* stitute for Applied Sociology.

Whetstone, W.R. (1987). Perceptions of Self-Care in East Germany: A Cross-Cultural Empirical Investigation. Journal of Advanced Nursing, 12, 167-176. 


\section{ANNEXES}

\section{Annex I Factoranalysis A.S.A.-A 7-8 Factors}

Table 1 Itemnumbers, Factor Loadings, Communalities

\begin{tabular}{|c|c|c|c|c|c|c|c|c|c|}
\hline & $\begin{array}{l}\text { Study I } \\
\text { Itam }\end{array}$ & $\begin{array}{l}\text { - UTI } \\
\text { loading }\end{array}$ & carrm. & $\begin{array}{l}\text { Study I- } \\
\text { Itgm }\end{array}$ & $\begin{array}{l}\text { MI } \\
\text { loading }\end{array}$ & ciommi. & $\begin{array}{l}\text { Study VI } \\
\text { Itiom }\end{array}$ & II & carm. \\
\hline \multirow[t]{4}{*}{ Factor 1} & 4 & .69 & .55 & 4 & .1. 59 & .64 & 2 & .82 & 89 \\
\hline & 18 & .69 & .59 & 10 & .68 & .50 & 19 & .79 & .70 \\
\hline & 10 & .64 & .46 & 18 & .63 & .57 & 3 & .54 & $* 41$ \\
\hline & 3 & .56 & *43 & 3 & "6 63 & .49 & g & .41 & 48 \\
\hline \multirow[t]{3}{*}{ Factor 2} & 23 & .78 & .64 & 23 & .79 & .65 & 18 & .72 & .58 \\
\hline & 11 & .89 & .56 & 20 & .74 & .60 & 4 & .69 & .52 \\
\hline & 20 & .68 & .55 & 11 & .70 & .54 & 12 & .50 & .69 \\
\hline \multirow[t]{4}{*}{ Factor 3} & 19 & .70 & $.5 B$ & 4 & .64 & .59 & 23 & .78 & .67 \\
\hline & 17 & .60 & .45 & 8 & .57 & .47 & 10 & .77 & .85 \\
\hline & 2 & .59 & .53 & 5 & .57 & .45 & 20 & .38 & .69 \\
\hline & 5 & .39 & .35 & 2 & .44 & .47 & & & \\
\hline \multirow[t]{5}{*}{ Factor 4} & 14 & .64 & .56 & 21 & .69 & .622 & 1 & .67 & .54 \\
\hline & 12 & .63 & .49 & 14 & .66 & .63 & 21 & .59 & .50 \\
\hline & 15 & .53 & .50 & 12 & .52 & .51 & 22 & .58 & .58 \\
\hline & 16 & .50 & .42 & 16 & .44 & .44 & 24 & $.4 B$ & .47 \\
\hline & & & & & & & 13 & -0.41 & .63 \\
\hline \multirow[t]{4}{*}{ Factor 5} & 6 & .78 & .65 & 17 & .70 & .66 & 16 & .63 & .70 \\
\hline & 24 & .66 & 62 & 19 & .67 & .62 & 14 & .59 & .56 \\
\hline & 7 & $=0.37$ & .48 & 15 & .42 & .51 & 15 & .50 & .44 \\
\hline & & & & 7 & .41 & .45 & 5 & -0.49 & .57 \\
\hline \multirow[t]{3}{*}{ Factor 6} & 22 & 4616 & 52 & 6 & .78 & $.66 \mathrm{i}$ & 6 & .70 & .70 \\
\hline & $\mathrm{g}$ & .59 & .51 & 24 & .69 & .62 & 11 & .616 & .70 \\
\hline & 21 & .52 & .58 & 93 & .51 & .54 & & & \\
\hline \multirow[t]{3}{*}{ Factor 7} & 1 & .64 & 60 & 9 & .24 & .62 & 7 & .86 & .76 \\
\hline & 13 & -0.61 & 83 & 22 & .59 & .51 & 12 & .55 & .69 \\
\hline & $a$ & .50 & 48 & & & & & & \\
\hline Factor 0 & & & & & & & 8 & .81 & 72 \\
\hline
\end{tabular}


Table 2 Itemnumbers, S.C.A. Power components and nature of items

\begin{tabular}{|c|c|c|c|c|c|c|c|c|c|}
\hline & $\begin{array}{l}\text { Study } \\
\text { Item } \\
4\end{array}$ & $\begin{array}{l}\text { I-VII } \\
\text { (pe) }\end{array}$ & $y=$ & $\begin{array}{l}\text { Study } \\
\text { Itam } \\
\text { \# }\end{array}$ & $\begin{array}{l}I-V I \\
(p e)\end{array}$ & $* / 1-$ & $\begin{array}{l}\text { Study } \\
\text { Itam } \\
\#\end{array}$ & $\begin{array}{l}\text { VIII } \\
\text { (pos) }\end{array}$ & $* /$ \\
\hline \multirow[t]{4}{*}{ Factor 1} & 3 & (III) & * & 3 & (III) & * & 3 & (III) & * \\
\hline & 10 & (WIII) & * & 10 & (VIII) & * & 2 & (IV) & - \\
\hline & 4 & $(x)$ & + & 4 & {$[x]$} & * & .9 & (II) & 4 \\
\hline & 10 & (VIII) & + & 18 & [VIII) & $*$ & 19 & {$[I]$} & * \\
\hline \multirow[t]{3}{*}{ Factor 2} & 23 & $(I x)$ & - & 23 & 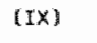 & - & 4 & $(x)$ & * \\
\hline & 20 & (WI) & - & 20 & $|I X|$ & + & 12 & (UIIT) & * \\
\hline & 11 & $(W I)$ & - & 11 & (WI) & - & 18 & (VIII) & $*$ \\
\hline \multirow[t]{4}{*}{ Factor 3} & 2 & (IW) & - & 2 & {$[I N]$} & - & 23 & $\{\mathbb{I X})$ & - \\
\hline & 5 & $(I X)$ & - & 5 & $(I X)$ & $*$ & 10 & (VITI) & $*$ \\
\hline & 17 & [V] & + & $\|$ & (IV) & 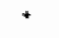 & 20 & $(I X)$ & + \\
\hline & 19 & (I) & $*$ & $\theta$ & $(x)$ & + & & & \\
\hline \multirow[t]{5}{*}{ Factor 4} & 12 & (VIII) & $*$ & 12 & (VIII) & $*$ & 22 & (VI) & 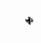 \\
\hline & 14 & (VII) & - & 14 & (VII) & - & 24 & $(I I I)$ & - \\
\hline & 16 & (VII) & + & 16 & $(\mathrm{VII})$ & $*$ & 13 & (II) & - \\
\hline & 15 & (I) & - & 21 & $(\mathbb{I})$ & - & 21 & (VII) & + \\
\hline & & & & & & & 1 & $(I V)$ & $*$ \\
\hline \multirow[t]{4}{*}{ Factor 5} & 7 & $(v)$ & + & 7 & $(w)$ & * & 16 & (VII) & + \\
\hline & 6 & (II) & - & 15 & (I) & - & 15 & (I) & - \\
\hline & 24 & (III) & - & 17 & (V) & $*$ & 14 & [VII] & - \\
\hline & & & & 19 & (I) & $*$ & 5 & $(I X)$ & - \\
\hline \multirow[t]{3}{*}{ Factor 6} & 22 & (VI) & + & 6 & (II) & - & 6 & (II) & - \\
\hline & 9 & (I) & + & 24 & (III $]$ & - & 11 & (VI) & - \\
\hline & 21 & [VII] & $*$ & 13 & (II) & - & & & \\
\hline \multirow[t]{3}{*}{ Factor 7} & 1 & (IV) & + & 9 & (I) & 4 & 7 & $(v)$ & + \\
\hline & 13 & (II) & - & 22 & (VI) & $*$ & 17 & (v) & \# \\
\hline & 3 & $(x)$ & + & & & & & & \\
\hline Factor 8 & & & & & & & $B$ & $(x)$ & * \\
\hline
\end{tabular}




\section{Table 3 Itemnumbers and Itemcontent}

Standy $I-V B$

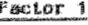

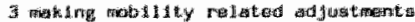

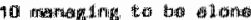

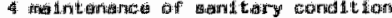

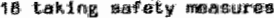

\section{Fantar 2}

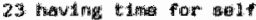

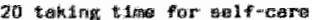

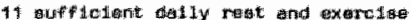

\section{Factor 3}

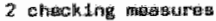

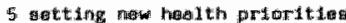

17 thangering old hablts

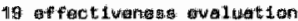

\section{Factor 4}

12 having edreing of friendsi

14 a king tor clantrication

183 obtatndmg madication dinfo

15 boidy m amilnait 1on

\section{Factor 5}

7 meking thovernt

6 suffictent anerzy

24 no notodisty Itmitetiona

\section{Foctor 6}

22 so: kiling halp

g appropriate foad hebits

21 gettung has ltih relatiod info

\section{Faetor 7}

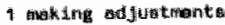

1.3 getting ancugh sime

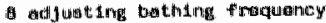

stivdy $1-$ wh

Deteor 1

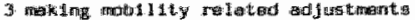

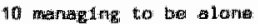

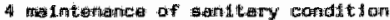

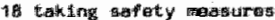

Fector 2

23 havdng the for 19

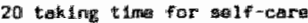

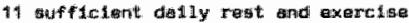

\section{Faetor 3}

2 check

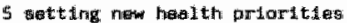

1 making adjustoments

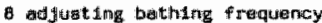

\section{Factor 4}

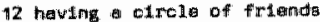

14 akling for clardif 1 cat lom

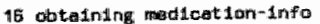

2.1 getting health rellated tonfo

\section{Factor 5}

7 seakinisi inproventent

15 body mexndination

13 chang ing old hablts

19 effoct I weness avoluation

\section{Factor}

B guffictent energy

24 no mob111ty Iimitations

19. gett1ng enough w19ep

\section{Factor?}

appropriate food thetorte

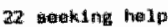

Sturdy $V$

\section{Factor}

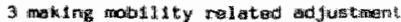

2 whecking measures

5 appromaty tood hoblt

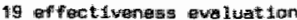

\section{Foctor 2}

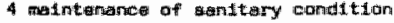
12 having cigche of triends

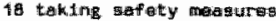

\section{Fractor 3}

23 heving tine for solf

10 managling to be ane

20 tiaking $t$ ing for self-care

\section{Factor 4}

22. gasking holp

24 no moblild ty 1 imal tat loms

13 gett1ng anough $1 \mathrm{gep}$

21 gatting health related info

1 making adgust:ming

Factor 5

ws obtaining medication-1nfo

15: body examination

14 asking for olarification

5. seiting new halith priorities

Factar 16

16. sufficient anengy

11 ouffictiont daliy rest and exareitie

\section{Factar $?$}

7 seakling Lsprovement

17 changlyig old habites

Factor:

a adyet lng bathlmg frequency 


\section{Annex II Factoranalysis A.S.A.-A 2 Factors}

Table 1 Itemnumbers, Factorloadings, communalities

\begin{tabular}{|c|c|c|c|c|c|c|c|c|c|}
\hline & \multicolumn{3}{|c|}{ Study I-W/II } & \multicolumn{3}{|c|}{ Study I - VI } & \multicolumn{3}{|c|}{ Study WII } \\
\hline & Iten & loading & corm. & Item \# & lowding & conm. & Itam \# & loading: & corm. \\
\hline \multirow[t]{17}{*}{ Factor 1} & 18 & .61 & .38 & 18 & .64 & .42 & 19 & .78 & .61 \\
\hline & 19 & .55 & .30 & 4 & .64 & .40 & 2 & .71 & .51 \\
\hline & 5 & .54 & .30 & 16 & .59 & .35 & 17 & .63 & .40 \\
\hline & 1 & .52 & .29 & $\mathrm{a}$ & .60 & .34 & 15 & 50 & .27 \\
\hline & B & .51 & .27 & 5 & .58 & .33 & 3 & .50 & .26 \\
\hline & 16 & .51 & .26 & 4 & .54 & .32 & 9 & .42 & .20 \\
\hline & 4 & .50 & .30 & 3 & .50 & .26 & 7 & .40 & .20 \\
\hline & 3 & .50 & .27 & $2 \%$ & .48 & .35 & 16 & .36 & .17 \\
\hline & 21 & .46 & .31 & 19 & .46 & .21 & 14 & .36 & .25 \\
\hline & 2 & .44 & .21 & 7 & .46 & .40 & $\theta$ & .33 & .12 \\
\hline & 17 & .39 & .25 & 10 & .42 & .19 & 1 & .31 & .19 \\
\hline & 14 & .34 & .16 & 2 & .36 & .18 & & & \\
\hline & 10 & .33 & .17 & 14 & .35 & .18 & & & \\
\hline & 15 & .32 & .12 & 12 & .33 & .12 & & & \\
\hline & 22 & .28 & .14 & 17 & .33 & .19 & & & \\
\hline & 12 & .27 & .07 & 15 & .32 & .13 & & & \\
\hline & 9 & .22 & .07 & & & & & & \\
\hline \multirow[t]{13}{*}{ Fector 2} & 23 & .64 & .43 & 23 & .67 & .47 & 20 & .59 & .35 \\
\hline & 20 & .62 & .42 & 20 & .67 & .48 & 10 & .56 & .32 \\
\hline & 11 & .59 & .38 & 11 & .66 & .44 & 23 & .55 & .30 \\
\hline & 13 & .52 & .27 & 13 & .52 & .29 & 6 & .54 & .30 \\
\hline & 6 & .51 & .26 & 6 & .47 & .22 & 4 & .50 & .25 \\
\hline & 7 & -0.44 & .39 & 24 & .25 & .09 & 22 & .49 & .25 \\
\hline & 24 & .28 & .088 & 22 & .25 & .41 & 11 & .43 & .21 \\
\hline & & & & 9 & .19 & .04 & 13 & .39 & .17 \\
\hline & & & & & & & 12 & $-3 \theta_{1}$ & .15. \\
\hline & & & & & & & 21 & .36 & 19 \\
\hline & & & & & & & 18 & .37 & .24 \\
\hline & & & & & & & 24 & .38 & .13 \\
\hline & & & & & & & 5 & .19 & .07 \\
\hline
\end{tabular}




\section{CURRICULUM VITAE}

Georges C.M. Evers was born in 1950 in Valkenswaard, The Netherlands. After completion of highschool he studied Greek, Latin, Classical Philosophy and Archeology at Catholic University of Nijmegen from 1968 till 1972. In that year he started his Nursing Studies and in 1976 he earned a Baccalaureate Degree in Nursing at Nijmegen School of Nursing. In 1977 he completed a Certificate Program in Educational Psychology at Nijmegen University. During four years a professional carreer as a nurse followed in Apeldoorn and Nijmegen. The last two years in a middle management position at the Thoracic Cardiac and Great Vessel Surgical Ward of Nijmegen University Hospital. In 1985 he earmed a Master's Degree in Nursing Science at University of Limburg, Maastricht. At this moment he is Assistant Professor at the Department of Nursing Science, Faculty of Health Sciences, University of Limburg. Since 1987 he has also taken a part-time position at the Dutch National Council for Health Affairs as Chair of the Permanent Committee for Health Professions. Furthermore he is Chair of the Scientific Nursing Council and member of the Scientific Medical Council of the C.B.O., Dutch W.H.O. collaborating Centre for Quality Assurance.

\section{CURRICULUM VITAE}

Georges C.M. Evers, in 1950 te Valkenswaard, Nederland, geboren studeerde na het behalen van het diploma Gymnasium A van 1968 tot 1972 Klassieke Taal en Letterkunde aan de Katholieke Universiteit te Nijmegen. Aan de Katholieke Hogere School voor Verpleegkundigen volgde hij van 1972 tot 1976 de Hogere Beroepsopleiding voor Verpleegkundigen H.B.O.-V. In 1977 behaalde hij een pedagogisch didactische aantekening aan de Katholieke Universiteit Nijmegen. Van 1976 tot en met 1980 volgde een verpleegkundige beroepsuitoefening in enkele Nederlandse ziekenhuizen, waarvan de laatste 2 jaar als leidinggevend verpleegkundige op de afdeling Thorax Hart en Vaatchirurgie van het St. Radboud Ziekenhuis te Nijmegen. In 1985 behaalde hij het doctoraal examen Gezondheidswetenschappen, afstudeerrichting Verplegingswetenschap aan de Rijksuniversiteit Limburg. Momenteel is hij als universitair docent verbonden aan de Vakgroep Verplegingswetenschap van de R.L. Sinds 1987 is hij voorts part-time werkzaam voor de Nationale Raad voor de Volksgezondheid als Voorzitter van de Kamer voor Beroepsvraagstukken. Daarenboven is hij Voorzitter van de Verpleegkundig Wetenschappelijke Raad en lid van de Medisch Wetenschappelijke Raad van het Centraal Begeleidings Orgaan intercollegiale toetsing, C.B.O. te Utrecht. 